\title{
FINITE-DIMENSIONAL IRREDUCIBLE REPRESENTATIONS OF TWISTED YANGIANS
}

\author{
Alexander Molev
}

\author{
Centre for Mathematics and its Applications \\ Australian National University \\ Canberra, ACT 0200, Australia \\ (e-mail: molev@pell.anu.edu.au)
}

November 1997

\begin{abstract}
We study quantized enveloping algebras called twisted Yangians. They are analogues of the Yangian $\mathrm{Y}(\mathfrak{g l}(N))$ for the classical Lie algebras of $B, C$, and $D$ series. The twisted Yangians are subalgebras in $\mathrm{Y}(\mathfrak{g l}(N))$ and coideals with respect to the coproduct in $\mathrm{Y}(\mathfrak{g l}(N))$. We give a complete description of their finite-dimensional irreducible representations. Every such representation is highest weight and we give necessary and sufficient conditions for an irreducible highest weight representation to be finite-dimensional. The result is analogous to Drinfeld's theorem for the ordinary Yangians. Its detailed proof for the $A$ series is also reproduced. For the simplest twisted Yangians we construct an explicit realization for each finitedimensional irreducible representation in tensor products of representations of the corresponding Lie algebras.
\end{abstract}

Mathematics Subject Classifications (1991). 17B37, 81R10 


\section{Introduction}

The Yangian $Y(\mathfrak{a})$ associated to a simple complex Lie algebra $\mathfrak{a}$ is a deformation of the universal enveloping algebra $\mathrm{U}(\mathfrak{a}[t])$ in the class of Hopf algebras [D1], [D2]. In the case of the $A$ series one can also define the Yangian $\mathrm{Y}(N)=\mathrm{Y}(\mathfrak{g l}(N))$ for the reductive complex Lie algebra $\mathfrak{a}=\mathfrak{g l}(N)$ (see, e.g., [TF]). The Yangian $\mathrm{Y}(\mathfrak{s l}(N))$ can be identified with a (Hopf) subalgebra in $\mathrm{Y}(\mathfrak{g l}(N))$. The Yangians are closely related to rational solutions of the Yang-Baxter equation [D1] and play the role of quantum symmetry groups of certain integrable systems; see, e.g., [BL], [KS1], [TU], [UK]. Finite-dimensional irreducible representations of the Yangians were classified by V. G. Drinfeld [D2]. In the case of $\mathfrak{a}=\mathfrak{g l}(2)$ this had been done by V. O. Tarasov $[\mathrm{T} 1]$, $[\mathrm{T} 2]$; see also $[\mathrm{K}] .{ }^{1}$ This result plays the main role in the proof in the general case. Representations of the Yangians were further studied in [CP1-CP3], [C1], [C2], [KR], [M2], [NT2].

For any $\mathfrak{a}$ the Yangian $\mathrm{Y}(\mathfrak{a})$ contains the universal enveloping algebra $U(\mathfrak{a})$. However, the $A$ series case seems to be exceptional since only in this case does there exists an algebra homomorphism $\mathrm{Y}(\mathfrak{a}) \rightarrow \mathrm{U}(\mathfrak{a})$ identical on $\mathrm{U}(\mathfrak{a})$. This homomorphism is important for applications of the 'quantum theory' to representations of the Lie algebra $\mathfrak{g l}(N)$ such as constructions of Laplace operators and characteristic identities [C2], [M4], [NT1]; Capelli identities [N1], [N2], [Ok].

Furthermore, as was shown by G. Olshanski [O1], the Yangian $\mathrm{Y}(N)$ can be constructed as a projective limit of $\mathfrak{g l}(M)$-centralizers in $\mathrm{U}(\mathfrak{g l}(N+M))$ as $M \rightarrow \infty$. It turned out that Olshanski's construction applied to other series of classical Lie algebras leads not to the corresponding Yangian $\mathrm{Y}(\mathfrak{a})$, but to different algebras which were called twisted Yangians [O2]; see also [S]. Defining relations for the twisted Yangian can be written in the form of a reflection-type equation (see (1.16) below) which allows one to use a special $R$-matrix technique for their study. Some other algebras associated to the reflection equation were studied in $[\mathrm{KK}],[\mathrm{KS} 2]$, [KJC].

Let $\mathfrak{g}(N)$ denote the orthogonal Lie algebra $\mathfrak{o}(N)$ or symplectic Lie algebra $\mathfrak{s p}(N)$. We regard $\mathfrak{g}(N)$ as a fixed point subalgebra in $\mathfrak{g l}(N)$ with respect to an involutive automorphism $\sigma$. The twisted Yangian is a deformation of the universal enveloping algebra $\mathrm{U}\left(\mathfrak{g l}(N)[t]^{\sigma}\right)$, where

$$
\mathfrak{g l}(N)[t]^{\sigma}=\{A(t) \in \mathfrak{g l}(N)[t] \mid \sigma(A(t))=A(-t)\} .
$$

We denote by $\mathrm{Y}^{+}(N)$ and $\mathrm{Y}^{-}(N)$ the twisted Yangians corresponding to the orthogonal and symplectic Lie algebras, respectively. Similarly to the $A$ series case the algebra $\mathrm{Y}^{ \pm}(N)$ contains the corresponding universal enveloping algebra $\mathrm{U}(\mathfrak{g}(N)$ ) and one has a natural algebra homomorphism $\mathrm{Y}^{ \pm}(N) \rightarrow \mathrm{U}(\mathfrak{g}(N))$ identical on $\mathrm{U}(\mathfrak{g}(N))$.

\footnotetext{
${ }^{1}$ In these papers the language of the quantum inverse scattering theory and integrable models was used. Representations of the Yangian Y(2) appear there in the form of the monodromy matrix for the XXX-model.
} 
A detailed description of the algebraic structure of the Yangian $\mathrm{Y}(N)$ and the twisted Yangians $\mathrm{Y}^{ \pm}(N)$ is contained in [MNO]. An explicit formula for the formal series (the Sklyanin determinant) whose coefficients generate the center of $\mathrm{Y}^{ \pm}(N)$ was given in [M3]. Bethe-type commutative subalgebras in $\mathrm{Y}^{ \pm}(N)$ were constructed in [NO]. Some applications of these results to the Laplace operators for the orthogonal and symplectic Lie algebras, characteristic identities and Capelli identities can be found in [M3], [M4], [MN].

In this paper we give a complete description of the finite-dimensional irreducible representations for all twisted Yangians. Our approach is very close to the $\mathfrak{g l}(N)$ case and we use some ideas and results in that case in our constructions.

Section 1 contains definitions and preliminary results on the Yangian $\mathrm{Y}(N)$ and the twisted Yangians $\mathrm{Y}^{ \pm}(N)$.

In Section 2 we give detailed proofs of Drinfeld's and Tarasov's theorems describing the finite-dimensional irreducible representations of $\mathrm{Y}(N)$. These representations can be constructed as follows. First, using the homomorphism $\mathrm{Y}(N) \rightarrow$ $\mathrm{U}(\mathfrak{g l}(N))$ one extends any finite-dimensional irreducible representation $L(\lambda)$ of the Lie algebra $\mathfrak{g l}(N)$ to the Yangian $\mathrm{Y}(N)$. Using the Hopf algebra structure on $\mathrm{Y}(N)$ one can consider representations of the form

$$
L\left(\lambda^{(1)}\right) \otimes \cdots \otimes L\left(\lambda^{(k)}\right) .
$$

Then any finite-dimensional irreducible representation of $\mathrm{Y}(\mathfrak{s l}(N))$ (or $\mathrm{Y}(N)$, up to tensoring with one-dimensional representations of the center of $\mathrm{Y}(N)$ ) can be realized as the irreducible quotient of the cyclic span in (0.1) of the tensor product of the highest weight vectors of the $L\left(\lambda^{(i)}\right)$.

In the case $N=2$ irreducible representations (0.1) constitute a complete list of finite-dimensional irreducible representation of $\mathrm{Y}(\mathfrak{s l}(2))$. A criterion of irreducibility of representation $(0.1)$ of $\mathrm{Y}(\mathfrak{s l}(2))$ in terms of 'strings' (subsets in $\mathbb{C}$ of the form $\{a, a+1, \ldots, b\})$ was given by Chari and Pressley [CP1], [CP3]. We give a proof of this result below (Corollary 2.11).

Alternatively, every finite-dimensional irreducible representation of $\mathrm{Y}(N)$ is highest weight (see Definition 2.1). It is isomorphic to a representation of the form $L(\lambda(u))$, where $\lambda(u)=\left(\lambda_{1}(u), \ldots, \lambda_{N}(u)\right)$ is a collection of formal series in $u^{-1}$. Moreover, given a set $\lambda(u)$ there exists a unique (up to an isomorphism) irreducible highest weight module $L(\lambda(u))$. Drinfeld's theorem [D2] (see Theorem 2.12 below) provides necessary and sufficient conditions for $L(\lambda(u))$ to be finite-dimensional.

In Sections 3-6 we give analogues of these descriptions for the twisted Yangians. Namely, using the homomorphism $\mathrm{Y}^{ \pm}(N) \rightarrow \mathrm{U}(\mathfrak{g}(N))$ we extend any finitedimensional irreducible representation $V(\mu)$ of the Lie algebra $\mathfrak{g}(N)$ to the twisted Yangian $\mathrm{Y}^{ \pm}(N)$. The subalgebra $\mathrm{Y}^{ \pm}(N) \subset \mathrm{Y}(N)$ is a left coideal,

$$
\Delta\left(\mathrm{Y}^{ \pm}(N)\right) \subset \mathrm{Y}(N) \otimes \mathrm{Y}^{ \pm}(N)
$$

and so, we may construct representations of $\mathrm{Y}^{ \pm}(N)$ of the form

$$
L\left(\lambda^{(1)}\right) \otimes \cdots \otimes L\left(\lambda^{(k)}\right) \otimes V(\mu) .
$$


As in the $\mathfrak{g l}(N)$-case, any finite-dimensional irreducible representation of $\mathrm{Y}^{ \pm}(N)$ (up to tensoring with one-dimensional representations of the center) can be realized as the irreducible quotient of the cyclic span in (0.3) of the tensor product of the highest weight vectors of the $L\left(\lambda^{(i)}\right)$ and $V(\mu)$.

On the other hand, we prove that any finite-dimensional irreducible representations of $\mathrm{Y}^{ \pm}(N)$ is highest weight, that is, isomorphic to a representation of the form $V(\mu(u)$ ) (see Definition 3.1), and we find necessary and sufficient conditions for $V(\mu(u))$ to be finite-dimensional; see Theorems 4.8, 5.9, 6.7 for the cases of $C$, $D, B$ series, respectively.

The key part of the proof is a detailed study of the simplest cases $\mathrm{Y}^{ \pm}(2)$ and $\mathrm{Y}^{+}(3)$. We use a modified Tarasov's argument [T1], [T2] and also use some ideas from [CP1], [CP3], as well as [Z1], [Z2] where representations of the super-Yangians were studied.

We give explicit realizations for all finite-dimensional irreducible representations of the simplest twisted Yangians $\mathrm{Y}^{-}(2)$ and $\mathrm{Y}^{+}(2)$ and prove analogues of ChariPressley's theorem for these algebras; see Corollaries 4.5, 4.7 and Corollaries 5.5, 5.6 , respectively.

The $B$ series case requires an extra care. We construct raising and lowering operators in a $\mathrm{Y}^{+}(3)$-module $V(\mu(u))$ which preserve the subspace of $\mathrm{Y}^{+}(2)$-singular vectors. To get the necessary conditions for $V(\mu(u))$ to be finite-dimensional we examine the weights of the $\mathrm{Y}^{+}(2)$-singular vectors and use the results for the case of $\mathrm{Y}^{+}(2)$.

Some of the results of this paper (mainly for the case of $\mathrm{Y}^{-}(2 n)$ ) were announced without complete proofs in [M1].

During this work I benefited from collaboration with G. Olshanski to whom I would like to express my sincere gratitude. I would also like to thank M. Nazarov, V. Tarasov and R. Zhang for valuable discussions.

\section{Definitions and preliminary results}

Our main reference for the algebraic structure of the Yangian $\mathrm{Y}(N)$ and the twisted Yangians $\mathrm{Y}^{ \pm}(N)$ is the expository paper [MNO]. In this section we give necessary definitions and formulate some results to be used in the subsequent sections.

We shall assume that given positive integer $N$, indices $i, j$ run through the set $\{-n, \ldots,-1,0,1, \ldots n\}$ if $N=2 n+1$ and run through the set $\{-n, \ldots,-1,1, \ldots n\}$ if $N=2 n$.

The Yangian $\mathrm{Y}(N)=\mathrm{Y}(\mathfrak{g l}(N))$ is the complex associative algebra with the generators $t_{i j}^{(1)}, t_{i j}^{(2)}, \ldots$ where $-n \leq i, j \leq n$, and the defining relations

$$
\left[t_{i j}^{(r+1)}, t_{k l}^{(s)}\right]-\left[t_{i j}^{(r)}, t_{k l}^{(s+1)}\right]=t_{k j}^{(r)} t_{i l}^{(s)}-t_{k j}^{(s)} t_{i l}^{(r)},
$$


where $r, s=0,1,2, \ldots$ and $t_{i j}^{(0)}:=\delta_{i j}$. For any $i, j$ define the formal power series

$$
t_{i j}(u)=\delta_{i j}+t_{i j}^{(1)} u^{-1}+t_{i j}^{(2)} u^{-2}+\cdots \in \mathrm{Y}(N)\left[\left[u^{-1}\right]\right] .
$$

Then the defining relations can be also written as follows:

$$
\left[t_{i j}(u), t_{k l}(v)\right]=\frac{1}{u-v}\left(t_{k j}(u) t_{i l}(v)-t_{k j}(v) t_{i l}(u)\right) .
$$

They also can be written as a single ternary relation (see, e.g., [MNO, Section 1]) for the T-matrix

$$
T(u):=\sum_{i, j} t_{i j}(u) \otimes E_{i j} \in \mathrm{Y}(N)\left[\left[u^{-1}\right]\right] \otimes \operatorname{End} \mathbb{C}^{N}
$$

where the $E_{i j}$ are the standard matrix units. It has the form

$$
R(u-v) T_{1}(u) T_{2}(v)=T_{2}(v) T_{1}(u) R(u-v) .
$$

Here both sides are regarded as elements of $\mathrm{Y}(N)\left[\left[u^{-1}\right]\right] \otimes \operatorname{End} \mathbb{C}^{N} \otimes$ End $\mathbb{C}^{N}$, the subindex of $T(u)$ indicates to which copy of End $\mathbb{C}^{N}$ this matrix corresponds; $R(u)=1-u^{-1} P$, where $P$ is the permutation operator in $\mathbb{C}^{N} \otimes \mathbb{C}^{N}$.

The following analogue of the Poincaré-Birkhoff-Witt theorem holds for the algebra $\mathrm{Y}(N)$ [MNO, Corollary 1.23]:

given an arbitrary linear order on the set of the generators $t_{i j}^{(r)}$, any element of the algebra $\mathrm{Y}(N)$ is uniquely written as a linear combination of ordered monomials in the generators.

In what follows we shall always assume that a linear order $\prec$ is chosen in such a way that

$$
t_{i_{1} j_{1}}^{\left(r_{1}\right)} \prec t_{i_{2} j_{2}}^{\left(r_{2}\right)} \prec t_{i_{3} j_{3}}^{\left(r_{3}\right)}
$$

as soon as $i_{1}>j_{1}, i_{2}=j_{2}$, and $i_{3}<j_{3}$.

We shall regard the set of the matrix units $\left\{E_{i j}\right\}$ as a basis of the Lie algebra $\mathfrak{g l}(N)$. The mapping

$$
E_{i j} \rightarrow t_{i j}^{(1)}
$$

defines an inclusion $\mathrm{U}(\mathfrak{g l}(N)) \hookrightarrow \mathrm{Y}(N)$.

The mapping

$$
t_{i j}(u) \mapsto \delta_{i j}+E_{i j} u^{-1}
$$

defines an algebra homomorphism $\mathrm{Y}(N) \rightarrow \mathrm{U}(\mathfrak{g l}(N))$.

The quantum determinant qdet $T(u)$ of the matrix $T(u)$ is a formal series in $u^{-1}$ with coefficients from $\mathrm{Y}(N)$ defined as follows:

$$
\operatorname{qdet} T(u)=\sum_{p \in \mathfrak{S}_{N}} \operatorname{sgn}(p) t_{p(-n),-n}(u) \cdots t_{p(n), n}(u-N+1),
$$


where $\mathfrak{S}_{N}$ is the group of permutations of the indices $\{-n, \ldots, n\}$.

The coefficients of the quantum determinant qdet $T(u)$ are algebraically independent generators of the center of the algebra $\mathrm{Y}(N)$.

Let us define the quantum comatrix $\widehat{T}(u)=\left(\widehat{t}_{i j}(u)\right)$ by the following formula:

$$
\text { qdet } T(u)=\widehat{T}(u) T(u-N+1) \text {. }
$$

Then $\widehat{t}_{i j}(u)$ equals $(-1)^{i+j}$ times the quantum determinant of the submatrix of $T(u)$ obtained by removing the $i$ th column and $j$ th row.

Each of the following mappings defines an antiautomorphism of the algebra $\mathrm{Y}(N)$ : change of sign in $u$

$$
\sigma: T(u) \rightarrow T(-u)
$$

a matrix transposition

$$
T(u) \rightarrow T^{t}(u)
$$

comatrix transformation

$$
T(u) \rightarrow \widehat{T}(u)
$$

The mapping

$$
T(u) \rightarrow T(u+a), \quad a \in \mathbb{C}
$$

and multiplication by a formal series $\varphi(u)=1+\varphi^{(1)} u^{-1}+\varphi^{(2)} u^{-2}+\cdots$ :

$$
T(u) \rightarrow \varphi(u) T(u)
$$

define automorphisms of $\mathrm{Y}(N)$.

The Yangian $\mathrm{Y}(\mathfrak{s l}(N))$ for the Lie algebra $\mathfrak{s l}(N)$ can be defined as the subalgebra of $\mathrm{Y}(N)$ consisting of the elements fixed by all automorphisms of the form (1.11). One has a tensor product decomposition

$$
\mathrm{Y}(N) \simeq \mathrm{Z}(N) \otimes \mathrm{Y}(\mathfrak{s l}(N))
$$

where $\mathrm{Z}(N)$ denotes the center of $\mathrm{Y}(N)$.

Using the automorphism of $\mathrm{Y}(N)$ of the form $T(u) \rightarrow T^{t}(-u)$, one can show that

$$
\operatorname{qdet} T(u)=\widehat{T}^{t}(u-1) T^{t}(u)
$$

Coproduct

$$
\Delta: \mathrm{Y}(N) \rightarrow \mathrm{Y}(N) \otimes \mathrm{Y}(N)
$$

is defined by the formula

$$
\Delta\left(t_{i j}(u)\right):=\sum_{a} t_{i a}(u) \otimes t_{a j}(u)
$$


Let us now define the twisted Yangians $\mathrm{Y}^{+}(N)$ and $\mathrm{Y}^{-}(N)$ corresponding to the orthogonal Lie algebra $\mathfrak{o}(N)$ and symplectic Lie algebra $\mathfrak{s p}(N)$, respectively. To consider both cases $\mathrm{Y}^{+}(N)$ and $\mathrm{Y}^{-}(N)$ simultaneously, it will be convenient to use the symbol $\theta_{i j}$ which is defined as follows:

$$
\theta_{i j}:= \begin{cases}1, & \text { in the orthogonal case; } \\ \operatorname{sgn}(i) \operatorname{sgn}(j), & \text { in the symplectic case. }\end{cases}
$$

Whenever the double sign \pm or $\mp$ occurs, the upper sign corresponds to the orthogonal case and the lower sign to the symplectic one. By $X \mapsto X^{t}$ we will denote the matrix transposition such that $\left(E_{i j}\right)^{t}=\theta_{i j} E_{-j,-i}$. Let us introduce the $S$-matrix $S(u)=\left(s_{i j}(u)\right)$ by setting $S(u):=T(u) T^{t}(-u)$, or, in terms of matrix elements,

$$
s_{i j}(u)=\sum_{a} \theta_{a j} t_{i a}(u) t_{-j,-a}(-u)
$$

Write

$$
s_{i j}(u)=\delta_{i j}+s_{i j}^{(1)} u^{-1}+s_{i j}^{(2)} u^{-2}+\cdots .
$$

The twisted Yangian $\mathrm{Y}^{ \pm}(N)$ is the subalgebra of $\mathrm{Y}(N)$ generated by the elements $s_{i j}^{(1)}, s_{i j}^{(2)}, \ldots$, where $-n \leq i, j \leq n$.

One can show that the $S$-matrix satisfies the following quaternary relation and symmetry relation (see [MNO, Section 3]):

$$
\begin{gathered}
R(u-v) S_{1}(u) R^{t}(-u-v) S_{2}(v)=S_{2}(v) R^{t}(-u-v) S_{1}(u) R(u-v), \\
S^{t}(-u)=S(u) \pm \frac{S(u)-S(-u)}{2 u} .
\end{gathered}
$$

Here we use the same notation as for the ternary relation $(1.2)$, where $R^{t}(u)$ is obtained from $R(u)$ by applying the transposition $t$ in either of the two copies of End $\mathbb{C}^{N}$.

Relations (1.16) are defining relations for the algebra $\mathrm{Y}^{ \pm}(N)$ and they can be rewritten in terms of the generating series $s_{i j}(u)$ as follows:

$$
\begin{aligned}
{\left[s_{i j}(u), s_{k l}(v)\right]=} & \frac{1}{u-v}\left(s_{k j}(u) s_{i l}(v)-s_{k j}(v) s_{i l}(u)\right) \\
& -\frac{1}{u+v}\left(\theta_{k,-j} s_{i,-k}(u) s_{-j, l}(v)-\theta_{i,-l} s_{k,-i}(v) s_{-l, j}(u)\right) \\
& +\frac{1}{u^{2}-v^{2}}\left(\theta_{i,-j} s_{k,-i}(u) s_{-j, l}(v)-\theta_{i,-j} s_{k,-i}(v) s_{-j, l}(u)\right)
\end{aligned}
$$

and

$$
\theta_{i j} s_{-j,-i}(-u)=s_{i j}(u) \pm \frac{s_{i j}(u)-s_{i j}(-u)}{2 u}
$$


The elements

$$
s_{i j}^{(2 k)}, \quad i+j \geq 0 ; \quad s_{i j}^{(2 k-1)}, \quad i+j>0 ; \quad k=1,2, \ldots
$$

in the case of $\mathrm{Y}^{+}(N)$, and the elements

$$
s_{i j}^{(2 k)}, \quad i+j>0 ; \quad s_{i j}^{(2 k-1)}, \quad i+j \geq 0 ; \quad k=1,2, \ldots
$$

in the case of $\mathrm{Y}^{-}(N)$, constitute a system of linearly independent generators. An analogue of the Poincaré-Birkhoff-Witt theorem for the algebra $\mathrm{Y}^{ \pm}(N)$ can be formulated as follows:

given an arbitrary linear order on the set of the above generators, any element of the algebra $\mathrm{Y}^{ \pm}(N)$ is uniquely written as a linear combination of ordered monomials in the generators;

see [MNO, Remark 3.14]. Similarly to the case of $\mathrm{Y}(N)$ we shall always assume that a linear order $\prec$ is chosen in such a way that

$$
s_{i_{1} j_{1}}^{\left(r_{1}\right)} \prec s_{i_{2} j_{2}}^{\left(r_{2}\right)} \prec s_{i_{3} j_{3}}^{\left(r_{3}\right)}
$$

as soon as $i_{1}>j_{1}, i_{2}=j_{2}$, and $i_{3}<j_{3}$.

Introduce the following elements of the Lie algebra $\mathfrak{g l}(N)$ :

$$
F_{i j}=E_{i j}-\theta_{i j} E_{-j,-i}, \quad-n \leq i, j \leq n .
$$

Denote by $\mathfrak{g}(N)$ the Lie subalgebra of $\mathfrak{g l}(N)$ spanned by the elements $F_{i j}$. Then $\mathfrak{g}(N)$ is isomorphic to $\mathfrak{o}(2 n)$ or $\mathfrak{s p}(2 n)$ if $N=2 n$, and to $\mathfrak{o}(2 n+1)$ if $N=2 n+1$.

The mapping

$$
F_{i j} \rightarrow s_{i j}^{(1)}
$$

defines an inclusion $\mathrm{U}(\mathfrak{g}(N)) \hookrightarrow \mathrm{Y}^{ \pm}(N)$.

The mapping

$$
s_{i j}(u) \mapsto \delta_{i j}+F_{i j}\left(u \pm \frac{1}{2}\right)^{-1}
$$

defines an algebra homomorphism $\mathrm{Y}^{ \pm}(N) \rightarrow \mathrm{U}(\mathfrak{g}(N))$.

There is an analogue of the quantum determinant for the twisted Yangians. It is denoted by sdet $S(u)$ and is called the Sklyanin determinant. This is a formal series in $u^{-1}$ and its coefficients generate the center of the algebra $\mathrm{Y}^{ \pm}(N)$. The Sklyanin determinant is related to the quantum determinant by the formula:

$$
\text { sdet } S(u)=\gamma_{N}(u) q \operatorname{qdet} T(u) q \operatorname{det} T(-u+N-1) \text {, }
$$

where $\gamma_{N}(u) \equiv 1$ for $\mathrm{Y}^{+}(N)$ and $\gamma_{N}(u)=(2 u+1) /(2 u-N+1)$ for $\mathrm{Y}^{-}(N)$. An explicit determinant-type expression for sdet $S(u)$ in terms of the generators $s_{i j}(u)$ was given in [M3]. 
Define the Sklyanin comatrix $\widehat{S}(u)=\left(\widehat{s}_{i j}(u)\right)$ by the following formula:

$$
\operatorname{sdet} S(u)=\widehat{S}(u) S(u-N+1) \text {. }
$$

An explicit expression for $\widehat{s}_{i j}(u)$ can also be found from [M3, Section 6].

The restriction of the antiautomorphism (1.7) to the subalgebra $\mathrm{Y}^{ \pm}(N)$ gives an antiautomorphism

$$
\sigma: S(u) \rightarrow S^{t}(u)
$$

We shall need the following result.

Proposition 1.1. The mapping

$$
S(u) \mapsto \gamma_{N}(u) \widehat{S}\left(-u+\frac{N}{2}-1\right),
$$

defines an automorphism of the algebra $\mathrm{Y}^{ \pm}(N)$.

Proof. Note that the mapping $T(u) \rightarrow \widetilde{T}(u)$ with

$$
\widetilde{T}(u)=\widehat{T}^{t}\left(u+\frac{N}{2}-1\right)=\operatorname{qdet} T\left(u+\frac{N}{2}\right)\left(T^{t}\left(u+\frac{N}{2}\right)\right)^{-1}
$$

defines an automorphism of the algebra $\mathrm{Y}(N)$. To see this, it suffices to present this map as a composition of antiautomorphisms of form (1.8), (1.9) and an automorphism of form (1.10). Denote by $\widetilde{S}(u)$ the image of the matrix $S(u)=T(u) T^{t}(-u)$ under this automorphism. That is,

$$
\widetilde{S}(u)=\widetilde{T}(u) \widetilde{T}^{t}(-u)=\widehat{T}^{t}\left(u+\frac{N}{2}-1\right) \widehat{T}\left(-u+\frac{N}{2}-1\right) .
$$

By (1.6) and (1.13) we have

$$
\begin{aligned}
& \widetilde{S}\left(-u+\frac{N}{2}-1\right) S(u-N+1)=\widehat{T}^{t}(-u+N-2) \widehat{T}(u) T(u-N+1) T^{t}(-u+N-1) \\
& \quad=\operatorname{qdet} T(u) \widehat{T}^{t}(-u+N-2) T^{t}(-u+N-1)=\operatorname{qdet} T(u) \operatorname{qdet} T(-u+N-1),
\end{aligned}
$$

which equals $\gamma_{N}^{-1}(u)$ sdet $S(u)$, by (1.24). Note that

$$
\gamma_{N}(u) \gamma_{N}\left(-u+\frac{N}{2}-1\right)=1
$$

and so, using (1.25) we obtain that

$$
\widetilde{S}(u)=\gamma_{N}(u) \widehat{S}\left(-u+\frac{N}{2}-1\right) .
$$


Thus, the subalgebra $\mathrm{Y}^{ \pm}(N) \subset \mathrm{Y}(N)$ is stable under the automorphism $T(u) \rightarrow$ $\widetilde{T}(u)$ and its restriction to $\mathrm{Y}^{ \pm}(N)$ yields the automorphism $(1.27)$ which completes the proof.

We shall use the following commutation relations between the matrix elements of the matrices $\widehat{S}(u)$ and $S(u)$ :

$$
\begin{aligned}
& {\left[\widehat{s}_{i j}(u), s_{k l}(v)\right]=-\frac{1}{u-v-N+1} \sum_{a}\left(\delta_{k j} \widehat{s}_{i a}(u) s_{a l}(v)-\delta_{i l} s_{k a}(v) \widehat{s}_{a j}(u)\right)} \\
& +\frac{1}{u+v+1} \sum_{a}\left(\delta_{i,-k} \theta_{a k} \widehat{s}_{-a, j}(u) s_{a l}(v)-\delta_{j,-l} \theta_{a l} s_{k a}(v) \widehat{s}_{i,-a}(u)\right) \\
& +\frac{1}{(u-v-N+1)(u+v+1)} \sum_{a}\left(\delta_{i,-k} \theta_{j k} \widehat{s}_{-j, a}(u) s_{a l}(v)-\delta_{j,-l} \theta_{i l} s_{k a}(v) \widehat{s}_{a,-i}(u)\right) .
\end{aligned}
$$

They easily follow from the quaternary relation; see (1.16). Indeed, inverting its both sides and multiplying from the left and right by $S_{2}(v)$ we obtain

$$
R^{t}(u+v-N) S_{1}^{-1}(u) R(v-u) S_{2}(v)=S_{2}(v) R(v-u) S_{1}^{-1}(u) R^{t}(u+v-N) .
$$

To get (1.28) rewrite this first in terms of the matrix elements of $S^{-1}(u)$ and $S(u)$, then use (1.25) and the centrality of the Sklyanin determinant in $\mathrm{Y}^{ \pm}(N)$. (In a slightly different form this calculation was performed in [MNO, Section 7]).

Multiplication by an even formal series $\psi(u)=1+\psi^{(2)} u^{-2}+\psi^{(4)} u^{-4}+\cdots$ defines an automorphism of $\mathrm{Y}^{ \pm}(N)$ :

$$
S(u) \rightarrow \psi(u) S(u)
$$

The special twisted Yangian $\mathrm{SY}^{ \pm}(N)$ is defined by the equality $\mathrm{SY}^{ \pm}(N)=$ $\mathrm{Y}(\mathfrak{s l}(N)) \cap \mathrm{Y}^{ \pm}(N)$. In other words, $\mathrm{SY}^{ \pm}(N)$ is the subalgebra of $\mathrm{Y}^{ \pm}(N)$ consisting of elements which are stable under all automorphisms of the form (1.29). One has the following tensor product decomposition analogous to (1.12):

$$
\mathrm{Y}^{ \pm}(N) \simeq \mathrm{Z}^{ \pm}(N) \otimes \mathrm{SY}^{ \pm}(N)
$$

where $\mathrm{Z}^{ \pm}(N)$ denotes the center of $\mathrm{Y}^{ \pm}(N)$.

The images of the generators $s_{i j}(u)$ under coproduct $\Delta$ are given by the formula

$$
\Delta\left(s_{i j}(u)\right)=\sum_{a, b} \theta_{b j} t_{i a}(u) t_{-j,-b}(-u) \otimes s_{a b}(u) .
$$

This implies that the subalgebra $\mathrm{Y}^{ \pm}(N) \subset \mathrm{Y}(N)$ is a left coideal; see (0.2). 


\section{Finite-dimensional irreducible representations of $\mathrm{Y}(N)$ and $\mathrm{Y}(\mathfrak{s l}(N))$}

For this section only we adopt a more standard enumeration of the generators $t_{i j}(u)$ of the Yangian $\mathrm{Y}(N)$ : we let the indices $i, j$ run through the set $\{1, \ldots, N\}$. All formulas from the previous section should be understood accordingly. In the following definitions we follow [D2] and [CP1-CP3].

Definition 2.1. A representation $L$ of the Yangian $\mathrm{Y}(N)$ is called highest weight if there exists a nonzero vector $\xi \in L$ such that $L$ is generated by $\xi$,

$$
t_{i j}(u) \xi=0 \quad \text { for } \quad 1 \leq i<j \leq N
$$

and

$$
t_{i i}(u) \xi=\lambda_{i}(u) \xi \quad \text { for } \quad 1 \leq i \leq N
$$

for some formal series $\lambda_{i}(u) \in 1+u^{-1} \mathbb{C}\left[\left[u^{-1}\right]\right]$. In this case the vector $\xi$ is called the highest weight vector of $L$ and the set $\lambda(u):=\left(\lambda_{1}(u), \ldots, \lambda_{N}(u)\right)$ is the highest weight of $L$.

Sometimes we shall also refer to $L$ as a representation with the highest weight $\lambda(u)$.

The definition of a lowest weight representation is obtained by replacing (2.1) with the relations

$$
t_{j i}(u) \xi=0 \quad \text { for } \quad 1 \leq i<j \leq N
$$

Definition 2.2. Let $\lambda(u)=\left(\lambda_{1}(u), \ldots, \lambda_{N}(u)\right)$ be any set of formal series where $\lambda_{i}(u) \in 1+u^{-1} \mathbb{C}\left[\left[u^{-1}\right]\right]$. The Verma module $M(\lambda(u))$ is the quotient of $\mathrm{Y}(N)$ by the left ideal generated by all the coefficients of the series $t_{i j}(u)$ for $1 \leq i<j \leq N$ and $t_{i i}(u)-\lambda_{i}(u)$ for $1 \leq i \leq N$.

The following properties of $M(\lambda(u))$ are immediate from the Poincaré-BirkhoffWitt theorem for $\mathrm{Y}(N)$. First, ordered monomials in the coefficients of the series $t_{i j}(u)$ with $i>j$ (assuming that (1.3) is satisfied) form a basis of $M(\lambda(u)$ ). The Verma module $M(\lambda(u))$ is obviously a representation of $\mathrm{Y}(N)$ with the highest weight $\lambda(u)$ and any representation with the highest weight $\lambda(u)$ is isomorphic to a quotient of $M(\lambda(u))$. Further, $M(\lambda(u))$ has a unique irreducible quotient which we denote by $L(\lambda(u))$. Indeed, the sum of all submodules of $M(\lambda(u))$ which do not contain the highest weight vector 1 is a unique maximal proper submodule $K$, and $L(\lambda(u))$ is the quotient $M(\lambda(u)) / K$. Moreover, if an irreducible $\mathrm{Y}(N)$-module $L$ contains a nonzero vector $\xi$ satisfying (2.1) and (2.2) then $L$ is isomorphic to $L(\lambda(u))$.

Next, using the inclusion (1.4) we may regard any $\mathrm{Y}(N)$-module as a $\mathfrak{g l}(N)$ module. Relations (1.1) imply that

$$
\left[E_{i j}, t_{k l}(u)\right]=\delta_{k j} t_{i l}(u)-\delta_{i l} t_{k j}(u)
$$


where we identify the elements $E_{i j} \in \mathfrak{g l}(N)$ with their images in $\mathrm{Y}(N)$. Let $\mathfrak{h}$ be the diagonal subalgebra in $\mathfrak{g l}(N)$ with the basis $E_{11}, \ldots, E_{N N}$ and let $\varepsilon_{1}, \ldots, \varepsilon_{N}$ be the dual basis in $\mathfrak{h}^{*}$. Choose the standard system of positive roots $R_{+}$for $\mathfrak{g l}(N)$ corresponding to the upper triangular Borel subalgebra. Then the elements $\alpha_{1}=\varepsilon_{1}-\varepsilon_{2}, \ldots, \alpha_{N-1}=\varepsilon_{N-1}-\varepsilon_{N}$ form a basis in $R_{+}$. If $L$ is a representation of $\mathrm{Y}(N)$ with the highest weight $\lambda(u)$, and $\xi$ is its highest weight vector, then $\xi$ is also a $\mathfrak{g l}(N)$-highest weight vector of the $\mathfrak{g l}(N)$-weight $\lambda=\left(\lambda_{1}, \ldots, \lambda_{N}\right)$ where $\lambda_{i}$ is the coefficient at $u^{-1}$ in the series $\lambda_{i}(u)$. By (2.3) all $\mathfrak{g l}(N)$-weights of $L$ have the form $\lambda-\omega$, where $\omega$ is a linear combination of the $\alpha_{i}$ with coefficients from $\mathbb{Z}_{+}$. Moreover, the subspace in $L$ of vectors of $\mathfrak{g l}(N)$-weight $\lambda$ if one-dimensional and spanned by $\xi$.

The following result is contained in [D2].

Theorem 2.3. Every finite-dimensional irreducible representation $L$ of $\mathrm{Y}(N)$ is highest weight. Moreover, L contains a unique (up to scalar multiples) highest weight vector.

Proof. The $\mathfrak{g l}(N)$-module $L$ admits a weight space decomposition

$$
L=\bigoplus_{\lambda \in \mathfrak{h}^{*}} L_{\lambda}, \quad L_{\lambda}=\left\{\eta \in L \mid E_{i i} \eta=\lambda_{i} \eta, \quad i=1, \ldots, N\right\}
$$

To see this, we note that by (2.3) the direct sum $L^{\prime}$ of all weight subspaces in $L$ is a $\mathrm{Y}(N)$-submodule. This submodule is nontrivial because $L$ is finite-dimensional and so it contains an eigenvector for the commutative subalgebra $\mathfrak{h}$. Since $L$ is irreducible as a $\mathrm{Y}(N)$-module we conclude that $L^{\prime}=L$.

Introduce the subspace in $L$

$$
L^{0}:=\left\{\eta \in L \mid t_{i j}(u) \eta=0 \quad \text { for } \quad 1 \leq i<j \leq N\right\}
$$

By (2.3) the weight space decomposition (2.4) induces that of $L^{0}$ :

$$
L^{0}=\bigoplus_{\lambda \in \mathfrak{h}^{*}} L_{\lambda}^{0}, \quad L_{\lambda}^{0}=L^{0} \cap L_{\lambda}
$$

Since $\mathfrak{g l}(N)$-module $L$ is finite-dimensional there exists a nonzero vector $\eta_{0} \in L$ of $\mathfrak{g l}(N)$-weight $\lambda^{\prime}$ such that $\lambda^{\prime}+\alpha$ is not a weight of $L$ for any $\alpha \in R_{+}$. We see from (2.3) that the vectors $t_{i j}^{(r)} \eta_{0}$ with $i<j$ and $r \geq 1$ have the $\mathfrak{g l}(N)$-weight $\lambda^{\prime}+\varepsilon_{i}-\varepsilon_{j}$ and therefore are equal to zero. This proves that the subspace $L^{0}$ is nontrivial.

Relations (1.1) and (2.3) imply that $\mathrm{Y}(N)$ can be equipped with a $\mathbb{Z}^{N}$-grading

$$
\mathrm{Y}(N)=\bigoplus_{\nu \in \mathbb{Z}^{N}} \mathrm{Y}(N)_{\nu}
$$


where

$$
\mathrm{Y}(N)_{\nu}=\left\{y \in \mathrm{Y}(N) \mid\left[E_{i i}, y\right]=\nu_{i} y, \quad i=1, \ldots, N\right\} .
$$

In particular, $\mathrm{Y}(N)_{0}$ is a subalgebra in $\mathrm{Y}(N)$.

Denote by $I$ and $I^{\prime}$ the left and right ideals in $\mathrm{Y}(N)$ respectively generated by the coefficients of the series $t_{i j}(u)$ and $t_{j i}(u)$, where $1 \leq i<j \leq N$. Set $I_{0}=\mathrm{Y}(N)_{0} \cap I$. Exactly as in the case of semisimple Lie algebras (see, e.g., [Di, Section 7.4]) one derives from the Poincaré-Birkhoff-Witt theorem for $\mathrm{Y}(N)$ that $I_{0}=I^{\prime} \cap \mathrm{Y}(N)_{0}$ and that $I_{0}$ is a two-sided ideal in $\mathrm{Y}(N)_{0}$. Moreover, $\mathrm{Y}(N)_{0}=\mathrm{Y} \oplus I_{0}$, where $\mathrm{Y}$ is the subspace in $\mathrm{Y}(N)_{0}$ with the basis formed by ordered monomials in the generators $t_{i i}^{(r)}$.

Consider the algebra $\mathcal{Y}=\mathrm{Y}(N)_{0} / I_{0}$. We see from $(1.1)$ that $\left[t_{i i}(u), t_{i i}(v)\right]=0$ and

$$
\left[t_{i i}(u), t_{j j}(v)\right]=\frac{1}{u-v}\left(t_{j i}(u) t_{i j}(v)-t_{j i}(v) t_{i j}(u)\right)
$$

So, if $i<j$ each commutator $\left[t_{i i}^{(r)}, t_{j j}^{(s)}\right]$ belongs to $I_{0}$. This implies that the algebra $\mathcal{Y}$ is commutative and freely generated by the elements $t_{i i}^{(r)} \bmod I_{0}$.

Further, we note that the subspace $L^{0}$ has a natural structure of $\mathrm{Y}(N)_{0}$-module and hence also of a $\mathcal{Y}$-module because $L^{0}$ is annihilated by $I_{0}$. Therefore, $L^{0}$ contains a nonzero vector $\xi$ which is a common eigenvector for all operators $t_{i i}^{(r)}$. So, $\xi$ generates a highest weight $\mathrm{Y}(N)$-submodule in $L$. Since $L$ is irreducible this submodule coincides with $L$ which proves that $L$ is highest weight.

Finally, if $\xi$ has $\mathfrak{g l}(N)$-weight $\lambda_{0}$ then nonzero weight subspaces in (2.6) can only correspond to the $\mathfrak{g l}(N)$-weights $\lambda$ of the form $\lambda_{0}-\omega$, where $\omega$ is a $\mathbb{Z}_{+}$-linear combination of the $\alpha_{i}$. Moreover, the subspace $L_{\lambda_{0}}^{0}$ is one-dimensional. However, each subspace $L_{\lambda}^{0}$ is $\mathcal{Y}$-invariant. If it is nonzero for a certain $\lambda \neq \lambda_{0}$ then a common eigenvector in $L_{\lambda}^{0}$ for all $t_{i i}^{(r)}$ generates a proper submodule is $L$, which makes a contradiction.

Remark 2.4. It follows from the proof that if $L$ is an irreducible highest weight representation of $\mathrm{Y}(N)$ (not necessary finite-dimensional) then the subspace (2.5) is one-dimensional and spanned by the highest weight vector $\xi$ of $L$.

Due to Theorem 2.3, to describe the finite-dimensional irreducible representations of $\mathrm{Y}(N)$ it suffices to find necessary and sufficient conditions for an irreducible highest weight representation $L(\lambda(u))$ to be finite-dimensional. The case of the simplest Yangian $\mathrm{Y}(2)$ plays a key role in the proof. We shall be assuming now that $N=2$.

The following result is due to V. O. Tarasov [T1], [T2].

Proposition 2.5. If $\operatorname{dim} L(\lambda(u))<\infty$ then there exists a formal series $\varphi(u) \in$ $1+u^{-1} \mathbb{C}\left[\left[u^{-1}\right]\right]$ such that $\varphi(u) \lambda_{1}(u)$ and $\varphi(u) \lambda_{2}(u)$ are polynomials in $u^{-1}$.

Proof. Let $\xi$ denote the highest weight vector of $L(\lambda(u))$. Since $L(\lambda(u))$ is finitedimensional there exists a nonnegative integer $k$ such that the vector $t_{21}^{(k+1)} \xi$ is a 
linear combination of $t_{21}^{(1)} \xi, \ldots, t_{21}^{(k)} \xi$. Take the minimum $k$ with this property. Let us show that then for any vector $t_{21}^{(r)} \xi$ with $r \geq k+1$ we have

$$
t_{21}^{(r)} \xi=a_{1}^{(r)} \xi_{1}+\cdots+a_{k}^{(r)} \xi_{k}
$$

for some complex coefficients $a_{i}^{(r)}$, where $\xi_{i}:=t_{21}^{(i)} \xi, i=1, \ldots, k$. Indeed, this is true for $r=k+1$ by our choice of $k$. Relations (1.1) imply that

$$
t_{11}^{(2)} t_{21}(u)=t_{21}(u)\left(t_{11}^{(2)}-t_{11}^{(1)}-u\right)+t_{21}^{(1)} t_{11}(u)
$$

and taking the coefficient at $u^{-p}$ we obtain

$$
t_{11}^{(2)} t_{21}^{(p)}=-t_{21}^{(p+1)}+t_{21}^{(1)} t_{11}^{(p)}+t_{21}^{(p)}\left(t_{11}^{(2)}-t_{11}^{(1)}\right)
$$

Write $\lambda_{1}(u)=1+\lambda_{1}^{(1)} u^{-1}+\lambda_{1}^{(2)} u^{-2}+\cdots$. Then by (2.9), for any $p \geq 1$,

$$
t_{21}^{(p+1)} \xi=-t_{11}^{(2)} t_{21}^{(p)} \xi+\left(\lambda_{1}^{(2)}-\lambda_{1}^{(1)}\right) t_{21}^{(p)} \xi+\lambda_{1}^{(p)} t_{21}^{(1)} \xi
$$

Hence, for $p=1, \ldots, k-1$ we have

$$
t_{11}^{(2)} \xi_{p}=\lambda_{1}^{(p)} \xi_{1}+\left(\lambda_{1}^{(2)}-\lambda_{1}^{(1)}\right) \xi_{p}-\xi_{p+1}
$$

and applying (2.7) with $r=k+1$, we get

$$
t_{11}^{(2)} \xi_{k}=\lambda_{1}^{(k)} \xi_{1}+\left(\lambda_{1}^{(2)}-\lambda_{1}^{(1)}\right) \xi_{k}-\left(a_{1}^{(k+1)} \xi_{1}+\cdots+a_{k}^{(k+1)} \xi_{k}\right) .
$$

Now (2.7) follows from (2.10)-(2.12) by an obvious induction on $r$.

So, rewriting (2.7) in terms of generating series we obtain

$$
t_{21}(u) \xi=a_{1}(u) \xi_{1}+\cdots+a_{k}(u) \xi_{k},
$$

where $a_{i}(u)=u^{-i}+a_{i}^{(k+1)} u^{-k-1}+\cdots$. Applying both sides of (2.8) to $\xi$ and using (2.13) we obtain

$$
\left(t_{11}^{(2)}-\lambda_{1}^{(2)}+\lambda_{1}^{(1)}+u\right)\left(a_{1}(u) \xi_{1}+\cdots+a_{k}(u) \xi_{k}\right)=\lambda_{1}(u) \xi_{1}
$$

Using (2.11) and (2.12) and taking the coefficient at $\xi_{p}, p=2, \ldots, k$ in this relation we get

$$
-a_{p-1}(u)+u a_{p}(u)-a_{p}^{(k+1)} a_{k}(u)=0 .
$$

This implies that for any $p=1, \ldots, k$ one has $a_{p}(u)=A_{p}(u) a_{k}(u)$ for a monic polynomial $A_{p}(u)$ of degree $k-p$ in $u$. Further, taking the coefficient at $\xi_{1}$ in $(2.14)$ we obtain $\lambda_{1}(u)=B(u) a_{k}(u)$ for a monic polynomial $B(u)$ of degree $k$ in $u$. 
Similarly, repeating this argument with the use of the relation

$$
t_{22}^{(2)} t_{21}(u)=t_{21}(u)\left(t_{22}^{(2)}+t_{22}^{(1)}+u\right)-t_{21}^{(1)} t_{22}(u)
$$

instead of (2.8) we obtain that $\lambda_{2}(u)=C(u) a_{k}(u)$ for a monic polynomial $C(u)$ of degree $k$ in $u$. Thus, the desired series is $\varphi(u)=\left(a_{k}(u) u^{k}\right)^{-1}$.

Given $\lambda=\left(\lambda_{1}, \ldots, \lambda_{N}\right) \in \mathfrak{h}^{*} \simeq \mathbb{C}^{N}$ we shall denote by $L(\lambda)$ the irreducible $\mathfrak{g l}(N)$-module with the highest weight $\lambda$ with respect to the basis $\left\{E_{11}, \ldots, E_{N N}\right\}$ in $\mathfrak{h}$. The representation $L(\lambda)$ is finite-dimensional if and only if $\lambda_{i}-\lambda_{i+1} \in \mathbb{Z}_{+}$for all $i=1, \ldots, N-1$. The homomorphism (1.5) allows us to consider any representation $L(\lambda)$ as a $\mathrm{Y}(N)$-module. It is obviously highest weight with $\lambda_{i}(u)=1+\lambda_{i} u^{-1}$, $i=1, \ldots, N$. The Hopf algebra structure on $\mathrm{Y}(N)$ (see (1.14)) allows one to naturally equip any tensor product $L_{1} \otimes L_{2}$ of $\mathrm{Y}(N)$-modules with a $\mathrm{Y}(N)$-action by the rule

$$
y \cdot\left(\xi_{1} \otimes \xi_{2}\right):=\Delta(y)\left(\xi_{1} \otimes \xi_{2}\right), \quad y \in \mathrm{Y}(N), \quad \xi_{i} \in L_{i}
$$

Let us now return to the case $N=2$. A basis in the irreducible $\mathfrak{g l}(2)$-module $L(\alpha, \beta)$ with the highest weight $(\alpha, \beta)$ is formed by the elements $\left(E_{21}\right)^{r} \xi$, where $\xi$ is the highest weight vector and $r=0, \ldots, \alpha-\beta$ if $\alpha-\beta \in \mathbb{Z}_{+}$; or $r \in \mathbb{Z}_{+}$otherwise. Using (2.15) we can construct representations of $\mathrm{Y}(2)$ of the form

$$
L=L\left(\alpha_{1}, \beta_{1}\right) \otimes \cdots \otimes L\left(\alpha_{k}, \beta_{k}\right) .
$$

In accordance with (1.14) the generators of $\mathrm{Y}(2)$ act by the rule

$$
t_{i j}(u)\left(\eta_{1} \otimes \cdots \otimes \eta_{k}\right)=\sum_{a_{1}, \ldots, a_{k-1}} t_{i a_{1}}(u) \eta_{1} \otimes t_{a_{1} a_{2}}(u) \eta_{2} \otimes \cdots \otimes t_{a_{k-1} j}(u) \eta_{k}
$$

where $\eta_{i} \in L\left(\alpha_{i}, \beta_{i}\right)$. By (1.5) all generators $t_{a b}^{(r)}$ with $r \geq 2$ act as zero operators in each $L\left(\alpha_{i}, \beta_{i}\right)$ and so, by (2.17) we have for any $\eta \in L$

$$
t_{i j}^{(r)} \eta=0, \quad \text { for } \quad r \geq k+1 .
$$

For a set of complex numbers $\left\{\alpha_{1}, \ldots, \alpha_{k}, \beta_{1}, \ldots, \beta_{k}\right\}$ we can find re-enumerations of the $\alpha_{i}$ and of the $\beta_{i}$ such that the following condition is satisfied:

for every $i=1, \ldots, k$ we have:

if the set $\left\{\alpha_{p}-\beta_{q} \mid i \leq p, q \leq k\right\} \cap \mathbb{Z}_{+}$is not empty

then $\alpha_{i}-\beta_{i}$ is its minimal element.

Indeed, choose first a minimal nonnegative integer difference among all differences $\alpha_{p}-\beta_{q}$ if it exists. Re-enumerating the indices if necessary, we may assume that this difference is $\alpha_{1}-\beta_{1}$. Then we proceed by induction, considering the differences $\alpha_{p}-\beta_{q}$ with $p, q \geq 2$ and so on.

The following proposition was proved in [T2]. 
Proposition 2.6. Let $L\left(\lambda_{1}(u), \lambda_{2}(u)\right)$ be an irreducible highest weight representation of $\mathrm{Y}(2)$ and $\lambda_{1}(u)$ and $\lambda_{2}(u)$ be polynomials of degree $\leq k$ in $u^{-1}$. Fix decompositions

$$
\begin{aligned}
& \lambda_{1}(u)=\left(1+\alpha_{1} u^{-1}\right) \cdots\left(1+\alpha_{k} u^{-1}\right), \\
& \lambda_{2}(u)=\left(1+\beta_{1} u^{-1}\right) \cdots\left(1+\beta_{k} u^{-1}\right)
\end{aligned}
$$

and assume that condition (2.19) is satisfied. Then $L\left(\lambda_{1}(u), \lambda_{2}(u)\right)$ is isomorphic to the tensor product (2.16).

Proof. Let $\xi_{i}$ be the highest weight vector of $L\left(\alpha_{i}, \beta_{i}\right)$. We have

$$
t_{11}(u) \xi_{i}=\left(1+\alpha_{i} u^{-1}\right) \xi_{i}, \quad t_{22}(u) \xi_{i}=\left(1+\beta_{i} u^{-1}\right) \xi_{i}, \quad \text { and } \quad t_{12}(u) \xi_{i}=0 .
$$

Therefore, the vector $\xi:=\xi_{1} \otimes \cdots \otimes \xi_{k}$ satisfies the relations

$$
t_{11}(u) \xi=\lambda_{1}(u) \xi, \quad t_{22}(u) \xi=\lambda_{2}(u) \xi \quad \text { and } \quad t_{12}(u) \xi=0
$$

which follows immediately from (2.17). Thus, the cyclic $\mathrm{Y}(2)$-span of the vector $\xi$ in $L$ is a highest weight module with the highest weight $\left(\lambda_{1}(u), \lambda_{2}(u)\right)$. Therefore, to prove Proposition 2.6 we only need to show that the representation $L$ is irreducible. We use induction on $k$.

For $k=1$ this is obvious, because $L(\alpha, \beta)$ is an irreducible $\mathfrak{g l}(2)$-module and hence also irreducible as a $\mathrm{Y}(2)$-module.

Suppose now that $k>1$. Let us prove first that any nonzero submodule $\widetilde{L}$ of $L$ contains the vector $\xi$. Note that $\widetilde{L}$ contains a nonzero vector $\eta$ such that $t_{12}(u) \eta=0$. Indeed, $\mathfrak{g l}(2)$-weights of $L$ have the form $\left(\sum_{i} \alpha_{i}-r, \sum_{i} \beta_{i}+r\right)$ with $r \in \mathbb{Z}_{+}$. Therefore, there exists a $\mathfrak{g l}(2)$-weight $\left(\mu_{1}, \mu_{2}\right)$ of $\widetilde{L}$ such that $\left(\mu_{1}+1, \mu_{2}-1\right)$ is not a weight. Then a vector $\eta$ of $\mathfrak{g l}(2)$-weight $\left(\mu_{1}, \mu_{2}\right)$ is annihilated by $t_{12}(u)$.

Write $\eta$ in the form

$$
\eta=\sum_{r=0}^{p}\left(E_{21}\right)^{r} \xi_{1} \otimes \eta_{r}
$$

with $p \geq 0$, where $\eta_{r} \in L\left(\alpha_{2}, \beta_{2}\right) \otimes \cdots \otimes L\left(\alpha_{k}, \beta_{k}\right)$; and if $\alpha_{1}-\beta_{1} \in \mathbb{Z}_{+}$then $p \leq \alpha_{1}-\beta_{1}$. We may assume that $\eta_{p} \neq 0$.

Applying $t_{12}(u)$ to $\eta$ with the use of (1.14) and (2.15) we get

$$
\sum_{r=0}^{p}\left(t_{11}(u)\left(E_{21}\right)^{r} \xi_{1} \otimes t_{12}(u) \eta_{r}+t_{12}(u)\left(E_{21}\right)^{r} \xi_{1} \otimes t_{22}(u) \eta_{r}\right)=0
$$

Using (1.5) and the commutation relations in $\mathfrak{g l}(2)$ we can write

$$
t_{11}(u)\left(E_{21}\right)^{r} \xi_{1}=\left(1+\left(\alpha_{1}-r\right) u^{-1}\right)\left(E_{21}\right)^{r} \xi_{1}
$$


and

$$
t_{12}(u)\left(E_{21}\right)^{r} \xi_{1}=u^{-1} r\left(\alpha_{1}-\beta_{1}-r+1\right)\left(E_{21}\right)^{r-1} \xi_{1} .
$$

Taking the coefficient at $\left(E_{21}\right)^{p} \xi_{1}$ in $(2.24)$ we get

$$
\left(1+\left(\alpha_{1}-p\right) u^{-1}\right) t_{12}(u) \eta_{p}=0 .
$$

This implies that $t_{12}(u) \eta_{p}=0$. On the other hand, by the induction hypotheses, the representation $L\left(\alpha_{2}, \beta_{2}\right) \otimes \cdots \otimes L\left(\alpha_{k}, \beta_{k}\right)$ is irreducible, and so

$$
\eta_{p}=\text { const } \cdot \xi_{2} \otimes \cdots \otimes \xi_{k}
$$

where 'const' is a nonzero constant; see Remark 2.4.

Suppose now that $p \geq 1$ and take the coefficient at $\left(E_{21}\right)^{p-1} \xi_{1}$ in $(2.24)$. By (2.25) and (2.26) we have

$$
\left(1+\left(\alpha_{1}-p+1\right) u^{-1}\right) t_{12}(u) \eta_{p-1}+u^{-1} p\left(\alpha_{1}-\beta_{1}-p+1\right) t_{22}(u) \eta_{p}=0 .
$$

Relations (2.27) and (2.17) imply that

$$
t_{22}(u) \eta_{p}=\left(1+\beta_{2} u^{-1}\right) \cdots\left(1+\beta_{k} u^{-1}\right) \eta_{p} .
$$

Multiply both sides of $(2.28)$ by $u^{k}$ :

$$
\left(u+\alpha_{1}-p+1\right) u^{k-1} t_{12}(u) \eta_{p-1}+p\left(\alpha_{1}-\beta_{1}-p+1\right) u^{k-1} t_{22}(u) \eta_{p}=0 .
$$

The operators $u^{k-1} t_{i j}(u)$ in $L\left(\alpha_{2}, \beta_{2}\right) \otimes \cdots \otimes L\left(\alpha_{k}, \beta_{k}\right)$ are polynomials in $u$ by (2.18) and we may put $u=-\alpha_{1}+p-1$ in (2.30). Taking into account (2.29) we get

$$
p\left(\alpha_{1}-\beta_{1}-p+1\right)\left(\alpha_{1}-\beta_{2}-p+1\right) \cdots\left(\alpha_{1}-\beta_{k}-p+1\right)=0 .
$$

However, this is impossible because of condition (2.19). Therefore, $p$ has to be equal to 0 and so, $\eta$ is a multiple of $\xi$.

To complete the proof we have to show that the submodule of $L$ generated by $\xi$ coincides with $L$.

Using the antiautomorphism (1.7) we equip the vector space $V^{*}$ dual to a $\mathrm{Y}(2)$ module $V$ with a structure of $\mathrm{Y}(2)$-module. Namely, set

$$
(y \cdot f)(v)=f(\sigma(y) v), \quad y \in \mathrm{Y}(2), \quad f \in V^{*}, \quad v \in V .
$$

Then one can easily see that $L\left(\alpha_{i}, \beta_{i}\right)^{*} \simeq L^{\prime}\left(-\alpha_{i},-\beta_{i}\right)$, where $L^{\prime}\left(-\alpha_{i},-\beta_{i}\right)$ is the lowest weight representation of $\mathfrak{g l}(2)$ with the lowest weight vector $\xi_{i}^{\prime}$ dual to $\xi_{i}$, of the weight $\left(-\alpha_{i},-\beta_{i}\right)$. This implies that

$$
L^{*} \simeq L^{\prime}\left(-\alpha_{1},-\beta_{1}\right) \otimes \cdots \otimes L^{\prime}\left(-\alpha_{k},-\beta_{k}\right) .
$$

Similarly to the case of representation $L$, any nonzero submodule of $L^{*}$ contains a nonzero vector $\eta^{\prime}$ such that $t_{21}(u) \eta^{\prime}=0$. Modifying the previous argument for lowest weight representations we prove that $\eta^{\prime}$ coincides (up to a nonzero factor) with the vector $\xi^{\prime}:=\xi_{1}^{\prime} \otimes \cdots \otimes \xi_{k}^{\prime}$. Now, if the submodule in $L$ generated by $\xi$ is proper, then its annihilator in $L^{*}$ is a nonzero submodule which does not contain $\xi^{\prime}$. Contradiction.

Proposition 2.6 allows one to get necessary and sufficient conditions for $L(\lambda(u))$ to be finite-dimensional. 
Proposition 2.7. Let $L(\lambda(u))$ be an irreducible representation of $\mathrm{Y}(2)$ with the highest weight $\lambda(u)=\left(\lambda_{1}(u), \lambda_{2}(u)\right)$. Then $L(\lambda(u))$ is finite-dimensional if and only if there exists a formal series $\varphi(u) \in 1+u^{-1} \mathbb{C}\left[\left[u^{-1}\right]\right]$ such that $\varphi(u) \lambda_{1}(u)$ and $\varphi(u) \lambda_{2}(u)$ are polynomials in $u^{-1}$ with decompositions

$$
\begin{aligned}
& \varphi(u) \lambda_{1}(u)=\left(1+\alpha_{1} u^{-1}\right) \cdots\left(1+\alpha_{k} u^{-1}\right), \\
& \varphi(u) \lambda_{2}(u)=\left(1+\beta_{1} u^{-1}\right) \cdots\left(1+\beta_{k} u^{-1}\right)
\end{aligned}
$$

and $\alpha_{i}-\beta_{i} \in \mathbb{Z}_{+}, i=1, \ldots, k$ for certain re-enumerations of the $\alpha_{i}$ and $\beta_{i}$.

Proof. Let $\operatorname{dim} L(\lambda(u))<\infty$. By Proposition 2.6, taking the composition of our representation with an automorphism of the form (1.11) we may assume that $(2.32)$ and (2.33) are satisfied for a certain series $\varphi(u)$. Re-enumerate the $\alpha_{i}$ and $\beta_{i}$ in such a way that condition (2.19) is satisfied. Now use Proposition 2.6. Since representation $(2.16)$ is finite-dimensional this gives the relations $\alpha_{i}-\beta_{i} \in \mathbb{Z}_{+}$for all $i=1, \ldots, k$.

Conversely, let (2.32) and (2.33) hold with $\alpha_{i}-\beta_{i} \in \mathbb{Z}_{+}, i=1, \ldots, k$. Then representation (2.16) is finite-dimensional and the composition of $L(\lambda(u))$ with the automorphism (1.11) corresponding to the series $\varphi(u)$ is isomorphic to the irreducible quotient of the $\mathrm{Y}(2)$-cyclic span in $L$ of the vector $\xi$; see (2.23). Therefore, $\operatorname{dim} L(\lambda(u))<\infty$.

The following reformulation of this result is due to V. G. Drinfeld [D2].

Theorem 2.8. The irreducible highest weight representation $L\left(\lambda_{1}(u), \lambda_{2}(u)\right)$ of $\mathrm{Y}(2)$ is finite-dimensional if and only if there exists a monic polynomial $P(u) \in \mathbb{C}[u]$ such that

$$
\frac{\lambda_{1}(u)}{\lambda_{2}(u)}=\frac{P(u+1)}{P(u)} .
$$

In this case $P(u)$ is unique.

Proof. If $\operatorname{dim} L\left(\lambda_{1}(u), \lambda_{2}(u)\right)<\infty$ then by Proposition 2.7 relations (2.32) and (2.33) hold for a certain series $\varphi(u)$. If $\alpha_{i}-\beta_{i} \in \mathbb{Z}_{+}$for all $i$ we put

$$
P(u)=\prod_{i=1}^{k}\left(u+\beta_{i}\right) \cdots\left(u+\alpha_{i}-1\right) .
$$

Conversely, suppose $(2.34)$ is satisfied for $P(u)=\left(u+\gamma_{1}\right) \cdots\left(u+\gamma_{s}\right)$. Define the polynomials

$$
\lambda_{1}^{\prime}(u)=\left(1+\left(\gamma_{1}+1\right) u^{-1}\right) \cdots\left(1+\left(\gamma_{s}+1\right) u^{-1}\right)
$$

and

$$
\lambda_{2}^{\prime}(u)=\left(1+\gamma_{1} u^{-1}\right) \cdots\left(1+\gamma_{s} u^{-1}\right) .
$$

By Proposition 2.7, the irreducible highest weight representation $L\left(\lambda_{1}^{\prime}(u), \lambda_{2}^{\prime}(u)\right)$ is finite-dimensional. However, $\lambda_{1}^{\prime}(u)$ and $\lambda_{2}^{\prime}(u)$ satisfy (2.34) and so, there exists 
an automorphism of $\mathrm{Y}(2)$ of the form (1.11) such that its composition with the representation $L\left(\lambda_{1}^{\prime}(u), \lambda_{2}^{\prime}(u)\right)$ is isomorphic to $L\left(\lambda_{1}(u), \lambda_{2}(u)\right)$. Thus, the latter is also finite-dimensional.

To complete the proof of the theorem, suppose that $Q(u)$ is another monic polynomial in $u$ and

$$
\frac{P(u+1)}{P(u)}=\frac{Q(u+1)}{Q(u)}
$$

Then $P(u) / Q(u)$ is periodic in $u$ which is only possible when $P(u)=Q(u)$.

The polynomial $P(u)$ defined by $(2.34)$ is called the Drinfeld polynomial; see also Corollary 2.13 below.

Relation (2.34) motivates the following notation which will be used in the sequel. For two formal series $\lambda_{1}(u)$ and $\lambda_{1}(u)$ in $u^{-1}$ we shall write

$$
\lambda_{1}(u) \rightarrow \lambda_{2}(u)
$$

if there exists a monic polynomial $P(u)$ in $u$ such that $(2.34)$ holds.

Due to the decomposition (1.12) we can establish a correspondence between representations of $\mathrm{Y}(N)$ and $\mathrm{Y}(\mathfrak{s l}(N))$ analogous to the correspondence between the representations of the Lie algebras $\mathfrak{g l}(N)$ and $\mathfrak{s l}(N)$. Consider the following similarity classes of finite-dimensional irreducible representations of $\mathrm{Y}(N)$ : two representations belong to the same class if one can be obtained from the other by the composition with an automorphism (1.11). Since the subalgebra $\mathrm{Y}(\mathfrak{s l}(N))$ is stable with respect to all automorphisms of the form (1.11), any representation from such a class, restricted to the subalgebra $\mathrm{Y}(\mathfrak{s l}(N))$ gives the same representation of $\mathrm{Y}(\mathfrak{s l}(N))$. Moreover, all finite-dimensional irreducible representations of $\mathrm{Y}(\mathfrak{s l}(N))$ can be obtained in this way.

In particular, using Theorem 2.8 we obtain a complete description of finitedimensional irreducible representations of the Yangian $\mathrm{Y}(\mathfrak{s l}(2))$.

Corollary 2.9. There is a one-to-one correspondence between finite-dimensional irreducible representations of the Yangian $\mathrm{Y}(\mathfrak{s l}(2))$ and monic polynomials in $u$. Every such representation is isomorphic to a representation of the form (2.16).

Proof. The first claim follows from the fact that the ratios $\lambda_{1}(u) / \lambda_{2}(u)$ take the same value for all representations $L\left(\lambda_{1}(u), \lambda_{2}(u)\right)$ of $\mathrm{Y}(2)$ of a given similarity class and hence by Theorem 2.8 the similarity classes are parameterized by monic polynomials in $u$.

Further, given a monic polynomial $P(u)$ we may find polynomials $\lambda_{1}(u)$ and $\lambda_{2}(u)$ in $u^{-1}$ such that relation (2.34) holds; see the proof of Theorem 2.8. Then by Proposition 2.6, the representation $L\left(\lambda_{1}(u), \lambda_{2}(u)\right)$ of $\mathrm{Y}(2)$ is isomorphic to a tensor product $(2.16)$ and its restriction to $\mathrm{Y}(\mathfrak{s l}(2))$ gives the finite-dimensional irreducible representation corresponding to $P(u)$. 
Remark 2.10. Tensoring $L\left(\lambda_{1}(u), \lambda_{2}(u)\right)$ by factors of the form $L(\gamma, \gamma)$ is equivalent to multiplying both $\lambda_{1}(u)$, and $\lambda_{2}(u)$ simultaneously by $\gamma(u)=1+\gamma u^{-1}$. However, the representations $L\left(\lambda_{1}(u), \lambda_{2}(u)\right)$ and $L\left(\gamma(u) \lambda_{1}(u), \gamma(u) \lambda_{2}(u)\right)$ of $\mathrm{Y}(2)$ belong to the same similarity class. Therefore, we might add to Corollary 2.9 the condition that in (2.16) all differences $\alpha_{i}-\beta_{i}$ are positive integers.

Since all finite-dimensional irreducible representation of $\mathrm{Y}(\mathfrak{s l}(2))$ have the form of (2.16) it is useful to have a criterion of irreducibility of such representations.

Following [CP1] we define the string corresponding to a pair of complex numbers $(\alpha, \beta)$ with $\alpha-\beta \in \mathbb{Z}_{+}$as the set

$$
S(\alpha, \beta)=\{\beta, \beta+1, \ldots, \alpha-1\} \subset \mathbb{C} .
$$

If $\alpha=\beta$ the set $S(\alpha, \beta)$ is considered to be empty. We say that two strings $S_{1}$ and $S_{2}$ are in general position if either

(i) $S_{1} \cup S_{2}$ is not a string, or

(ii) $S_{1} \subseteq S_{2}$, or $S_{2} \subseteq S_{1}$.

The following result is contained in [CP1] and [CP3].

Corollary 2.11. Consider the representation $L$ of $\mathrm{Y}(2)$ or $\mathrm{Y}(\mathfrak{s l}(2))$ given by (2.16) where all the differences $\alpha_{i}-\beta_{i}$ are nonnegative integers. Then $L$ is irreducible if and only if the strings $S\left(\alpha_{1}, \beta_{1}\right), \ldots, S\left(\alpha_{k}, \beta_{k}\right)$ are pairwise in general position.

Proof. Suppose that the strings are pairwise in general position and assume first that $\alpha_{1}-\beta_{1} \leq \cdots \leq \alpha_{k}-\beta_{k}$. Then one easily checks that (2.19) holds and so, by Proposition 2.6 representation (2.16) is irreducible. To complete the proof of the 'if' part note that if representation (2.16) is irreducible, then any permutation of the tensor factors gives an isomorphic representation $L^{\prime}$. Indeed, the submodule in $L^{\prime}$ generated by the tensor product of the highest weight vectors of the $L\left(\alpha_{i}, \beta_{i}\right)$ is highest weight with the same $\lambda_{1}(u)$ and $\lambda_{2}(u)$ given by (2.20) and (2.21). Therefore, $L$ is isomorphic to a subquotient of $L^{\prime}$. As $L$ and $L^{\prime}$ have the same dimension, they have to be isomorphic.

Conversely, let $k=2$ and $L\left(\alpha_{1}, \beta_{1}\right) \otimes L\left(\alpha_{2}, \beta_{2}\right)$ be irreducible. Suppose that the strings $S\left(\alpha_{1}, \beta_{1}\right)$ and $S\left(\alpha_{2}, \beta_{2}\right)$ are not in general position. Then $\alpha_{2}-\beta_{1}$, $\alpha_{1}-\beta_{2} \in \mathbb{Z}_{+}$and either both of $\beta_{1}-\beta_{2}$ and $\alpha_{1}-\alpha_{2}$, or both of $\beta_{2}-\beta_{1}$ and $\alpha_{2}-\alpha_{1}$ are positive integers. The strings $S\left(\alpha_{1}, \beta_{2}\right)$ and $S\left(\alpha_{2}, \beta_{1}\right)$ are in general position and so, the representation $L\left(\alpha_{1}, \beta_{2}\right) \otimes L\left(\alpha_{2}, \beta_{1}\right)$ is irreducible. Since it has the same highest weight as the irreducible representation $L\left(\alpha_{1}, \beta_{1}\right) \otimes L\left(\alpha_{2}, \beta_{2}\right)$ these two representations have to be isomorphic and, in particular, have the same dimension:

$$
\left(\alpha_{1}-\beta_{1}+1\right)\left(\alpha_{2}-\beta_{2}+1\right)=\left(\alpha_{1}-\beta_{2}+1\right)\left(\alpha_{2}-\beta_{1}+1\right) .
$$

This implies that $\left(\alpha_{1}-\alpha_{2}\right)\left(\beta_{1}-\beta_{2}\right)=0$, which is impossible. 
Now consider the general case. Suppose that $L$ is irreducible but two strings $S\left(\alpha_{i}, \beta_{i}\right)$ and $S\left(\alpha_{j}, \beta_{j}\right)$ are not in general position. Permuting the factors in (2.16) if necessary, we may assume that $i$ and $j$ are adjacent. However, the representation $L\left(\alpha_{i}, \beta_{i}\right) \otimes L\left(\alpha_{j}, \beta_{j}\right)$ is reducible. Contradiction.

We are now able to prove Drinfeld's classification theorem for the Yangian $\mathrm{Y}(N)$, $N$ is an arbitrary positive integer; see [D2]. Theorem 2.3 together with the following theorem give a complete description of finite-dimensional irreducible representations of $\mathrm{Y}(N)$. We use notation (2.35).

Theorem 2.12. The irreducible highest weight representation $L(\lambda(u)), \lambda(u)=$ $\left(\lambda_{1}(u), \ldots, \lambda_{N}(u)\right)$ of $\mathrm{Y}(N)$ is finite-dimensional if and only if the following relation holds:

$$
\lambda_{1}(u) \rightarrow \lambda_{2}(u) \rightarrow \cdots \rightarrow \lambda_{N}(u) .
$$

Proof. Suppose that $\operatorname{dim} L(\lambda(u))<\infty$. By the Poincaré-Birkhoff-Witt theorem for $\mathrm{Y}(N)$, for any $i \in\{1, \ldots, N-1\}$ the subalgebra in $\mathrm{Y}(N)$ generated by the coefficients of the series $t_{i i}(u), t_{i, i+1}(u), t_{i+1, i}(u), t_{i+1, i+1}(u)$ is isomorphic to $\mathrm{Y}(2)$. The cyclic span of the highest weight vector of $L(\lambda(u))$ with respect to this subalgebra is a representation with the highest weight $\left(\lambda_{i}(u), \lambda_{i+1}(u)\right)$. Its irreducible quotient is finite-dimensional and so, applying Theorem 2.8 we get (2.36).

Conversely, let

$$
\frac{\lambda_{i}(u)}{\lambda_{i+1}(u)}=\frac{P_{i}(u+1)}{P_{i}(u)}
$$

for certain polynomials $P_{i}(u), i=1, \ldots, N-1$ given by

$$
P_{i}(u)=\left(u+\gamma_{1}^{(i)}\right) \cdots\left(u+\gamma_{s_{i}}^{(i)}\right)
$$

Consider first the irreducible highest weight representation $L\left(\lambda^{\prime}(u)\right)$ of $\mathrm{Y}(N)$ with the highest weight $\lambda^{\prime}(u)=\left(\lambda_{1}^{\prime}(u), \ldots, \lambda_{N}^{\prime}(u)\right)$, where

$$
\begin{aligned}
\lambda_{i}^{\prime}(u) & =\prod_{a=1}^{i-1}\left(1+\gamma_{1}^{(a)} u^{-1}\right) \cdots\left(1+\gamma_{s_{a}}^{(a)} u^{-1}\right) \\
& \times \prod_{a=i}^{N-1}\left(1+\left(\gamma_{1}^{(a)}+1\right) u^{-1}\right) \cdots\left(1+\left(\gamma_{s_{a}}^{(a)}+1\right) u^{-1}\right) .
\end{aligned}
$$

These polynomials have the form

$$
\lambda_{i}^{\prime}(u)=\left(1+\alpha_{1}^{(i)} u^{-1}\right) \cdots\left(1+\alpha_{k}^{(i)} u^{-1}\right)
$$

with $k=\sum s_{i}$ and the condition $\alpha_{r}^{(i)}-\alpha_{r}^{(i+1)} \in \mathbb{Z}_{+}$is satisfied for each $r=1, \ldots, k$ and $i=1, \ldots, N-1$. Consider the tensor product

$$
L\left(\alpha_{1}\right) \otimes \cdots \otimes L\left(\alpha_{k}\right)
$$


where $L\left(\alpha_{r}\right)$ is the irreducible representation of $\mathfrak{g l}(N)$ with the highest weight $\alpha_{r}=\left(\alpha_{r}^{(1)}, \ldots, \alpha_{r}^{(N)}\right)$. The generators $t_{i j}(u)$ act in representation $(2.38)$ by the rule (2.17). So, the tensor product of the highest weight vectors of the $L\left(\alpha_{r}\right)$ generates a representation of $\mathrm{Y}(N)$ with the highest weight $\lambda^{\prime}(u)$. Hence, its irreducible quotient is isomorphic to $L\left(\lambda^{\prime}(u)\right)$. Representation (2.38) is finite-dimensional, and therefore $L\left(\lambda^{\prime}(u)\right)$ is.

Finally, note that the $\lambda_{i}^{\prime}(u)$ satisfy $(2.37)$ and so, there exists an automorphism of $\mathrm{Y}(N)$ of the form (1.11) such that its composition with the representation $L\left(\lambda^{\prime}(u)\right)$ is isomorphic to $L(\lambda(u))$ which completes the proof.

We conclude this section with a description of finite-dimensional irreducible representations of the Yangian $\mathrm{Y}(\mathfrak{s l}(N))$ implied by Theorem 2.12; see [D2].

Corollary 2.13. There is a one-to-one correspondence between finite-dimensional irreducible representations of the Yangian $\mathrm{Y}(\mathfrak{s l}(N))$ and the families of monic polynomials $\left\{P_{1}(u), \ldots, P_{N-1}(u)\right\}$ in $u$. Every such representation is isomorphic to a subquotient of a representation of the form (2.38).

The $P_{i}(u)$ are called the Drinfeld polynomials associated to the representation $L(\lambda(u))$ of $\mathrm{Y}(\mathfrak{s l}(N))$. Note that our definition of $P_{i}(u)$ differs from that of [D2] by a shift in $u$.

Remark 2.14. Contrary to the case $N=2$, it is not true for $N \geq 3$ that every finite-dimensional irreducible representation of $\mathrm{Y}(\mathfrak{s l}(N))$ is isomorphic to a tensor product (2.38). However, this is true for 'generic' representations; see, e.g., [KR], [M2], [NT2].

\section{Highest weight representations of the twisted Yangians}

Here we define highest weight representations of the algebras $\mathrm{Y}^{ \pm}(N)$ and prove an analogue of Theorem 2.3 for $\mathrm{Y}^{ \pm}(N)$. We also find some necessary conditions for an irreducible highest weight representation to be finite-dimensional and construct a family of representations of $\mathrm{Y}^{ \pm}(N)$.

From now on till the end of the paper we shall be assuming that the generators $t_{i j}(u)$ of $\mathrm{Y}(N)$ as well as the basis elements $E_{i j}$ of the Lie algebra $\mathfrak{g l}(N)$ are parameterized by the indices $i, j \in\{-n,-n+1, \ldots, n\}$, where $n=[N / 2]$ and the index 0 is skipped in $N=2 n$ (see Section 1$)$.

Definition 3.1. A representation $V$ of the twisted Yangian $\mathrm{Y}^{ \pm}(N)$ is called highest weight if there exists a nonzero vector $\xi \in V$ such that $V$ is generated by $\xi$,

$$
s_{i j}(u) \xi=0 \quad \text { for } \quad-n \leq i<j \leq n,
$$

and

$$
s_{i i}(u) \xi=\mu_{i}(u) \xi
$$


for some formal series $\mu_{i}(u) \in 1+u^{-1} \mathbb{C}\left[\left[u^{-1}\right]\right]$, where $i=1, \ldots, n$ if $N=2 n$ and $i=0, \ldots, n$ if $N=2 n+1$. In this case the vector $\xi$ is called the highest weight vector of $V$ and the set $\mu(u):=\left(\mu_{1}(u), \ldots, \mu_{n}(u)\right)$ or $\mu(u):=\left(\mu_{0}(u), \ldots, \mu_{n}(u)\right)$ is the highest weight of $V$.

Due to the symmetry relation (1.18), the vector $\xi$ is also an eigenvector for the generators $s_{-i,-i}(u), i=1, \ldots, n$. Moreover, if $N=2 n+1$ the component $\mu_{0}(u)$ of the highest weight has to be even: $\mu_{0}(u)=\mu_{0}(-u)$.

If we replace (3.1) by the relations

$$
s_{j i}(u) \xi=0 \quad \text { for } \quad-n \leq i<j \leq n .
$$

we get the definition of a lowest weight representation.

Definition 3.2. Let $\mu(u)=\left(\mu_{1}(u), \ldots, \mu_{n}(u)\right)$ or $\mu(u)=\left(\mu_{0}(u), \ldots, \mu_{n}(u)\right)$ be any set of formal series where $\mu_{i}(u) \in 1+u^{-1} \mathbb{C}\left[\left[u^{-1}\right]\right]$ for $i=1, \ldots, n$ and $\mu_{0}(u) \in$ $1+u^{-2} \mathbb{C}\left[\left[u^{-2}\right]\right]$. The Verma module $M(\mu(u))$ is the quotient of $\mathrm{Y}^{ \pm}(N)$ by the left ideal generated by all the coefficients of the series $s_{i j}(u)$ for $-n \leq i<j \leq n$ and $s_{i i}(u)-\mu_{i}(u)$ for $i=1, \ldots, n$ or $i=0, \ldots, n$, respectively.

The following properties of $M(\mu(u))$ are immediate from the Poincaré-BirkhoffWitt theorem for $\mathrm{Y}^{ \pm}(N)$ (see Section 1). First, ordered monomials in the generators (1.19) or (1.20) with the additional condition $i>j$ (assuming that (1.21) is satisfied) form a basis of $M(\mu(u))$. Further, $M(\mu(u))$ is a representation of $\mathrm{Y}^{ \pm}(N)$ with the highest weight $\mu(u)$ and any representation of the highest weight $\mu(u)$ is isomorphic to a quotient of $M(\mu(u))$. The module $M(\mu(u))$ has a unique irreducible quotient which we denote by $V(\mu(u))$. Moreover, if an irreducible $\mathrm{Y}^{ \pm}(N)$-module $V$ contains a nonzero vector $\xi$ satisfying (3.1) and (3.2) then $V$ is isomorphic to $V(\mu(u))$.

Using the inclusion (1.22) we may regard any $\mathrm{Y}^{ \pm}(N)$-module as a $\mathfrak{g}(N)$-module. Relations (1.17) imply that

$$
\left[F_{i j}, s_{k l}(u)\right]=\delta_{k j} s_{i l}(u)-\delta_{i l} s_{k j}(u)-\theta_{k,-j} \delta_{i,-k} s_{-j, l}(u)+\theta_{i,-l} \delta_{-l, j} s_{k,-i}(u),
$$

where we identify the elements $F_{i j} \in \mathfrak{g}(N)$ with their images in $\mathrm{Y}^{ \pm}(N)$. Set $\mathfrak{h}=$ span of $\left\{F_{11}, \ldots, F_{n n}\right\}$. Then $\mathfrak{h}$ is a Cartan subalgebra in $\mathfrak{g}(N)$. Choose the standard system of positive roots $R_{+}$for $\mathfrak{g}(N)$ with respect to $\mathfrak{h}$ corresponding to the upper triangular Borel subalgebra and let $\alpha_{1}, \ldots, \alpha_{n}$ be a basis in $R_{+}$. If $V$ is a representation of $\mathrm{Y}^{ \pm}(N)$ with the highest weight $\mu(u)$ then its highest weight vector $\xi$ is also a $\mathfrak{g}(N)$-highest weight vector of the $\mathfrak{g}(N)$-weight $\mu=\left(\mu_{1}, \ldots, \mu_{n}\right)$ where $\mu_{i}$ is the coefficient at $u^{-1}$ in the series $\mu_{i}(u)$. By $(3.3)$ all $\mathfrak{g}(N)$-weights of $V$ have the form $\mu-\omega$, where $\omega$ is a $\mathbb{Z}_{+}$-linear combination of the $\alpha_{i}$. Moreover, the subspace in $V$ of vectors of $\mathfrak{g}(N)$-weight $\mu$ if one-dimensional and spanned by $\xi$. 
Theorem 3.3. Every finite-dimensional irreducible representation $V$ of $\mathrm{Y}^{ \pm}(N)$ is highest weight. Moreover, $V$ contains a unique (up to scalar multiples) highest weight vector.

Proof. The proof follows the same steps as that of Theorem 2.3. We set

$$
V^{0}:=\left\{\eta \in V \mid s_{i j}(u) \eta=0 \quad \text { for } \quad-n \leq i<j \leq n\right\} .
$$

By (3.3) we have the weight space decomposition

$$
V^{0}=\bigoplus_{\mu \in \mathfrak{h}^{*}} V_{\mu}^{0}
$$

where

$$
V_{\mu}^{0}=\left\{\eta \in V^{0} \mid F_{i i} \eta=\mu_{i} \eta\right\}, \quad \mu_{i}=\mu\left(F_{i i}\right) .
$$

Introduce the $\mathfrak{h}$-centralizer in $\mathrm{Y}^{ \pm}(N)$ :

$$
\mathrm{Y}^{ \pm}(N)_{0}=\left\{y \in \mathrm{Y}^{ \pm}(N) \mid\left[F_{i i}, y\right]=0 \text { for all } i\right\} .
$$

Denote by $I$ the left ideal in $\mathrm{Y}^{ \pm}(N)$ generated by the coefficients of the series $s_{i j}(u)$, where $-n \leq i<j \leq n$ and set $I_{0}=\mathrm{Y}^{ \pm}(N)_{0} \cap I$. We derive from the Poincaré-Birkhoff-Witt theorem for $\mathrm{Y}^{ \pm}(N)$ that $I_{0}$ is a two-sided ideal in $\mathrm{Y}^{ \pm}(N)_{0}$. Next, using (1.17) we verify that the algebra $\mathcal{Y}:=\mathrm{Y}^{ \pm}(N)_{0} / I_{0}$ is commutative and generated by the elements $s_{i i}^{(r)} \bmod I_{0}$.

Further, $V^{0}$ is a nonzero $\mathcal{Y}$-module (cf. the proof of Theorem 2.3) and the weight spaces $V_{\mu}^{0}$ are $\mathcal{Y}$-invariant. If $V_{\mu_{0}}^{0} \neq 0$ for a certain $\mu_{0}$ then there exists a nonzero element $\xi \in V_{\mu_{0}}^{0}$ which is a common eigenvector for the generators $s_{i i}^{(r)}$. So, $\xi$ generates a highest weight $\mathrm{Y}^{ \pm}(N)$-submodule in $V$. Since $V$ is irreducible this submodule coincides with $V$ which proves that $V$ is highest weight.

Finally, the subspace $V_{\mu_{0}}^{0}$ is one-dimensional and nonzero weight subspaces in (3.5) can only correspond to the $\mathfrak{g}(N)$-weights $\mu$ of the form $\mu_{0}-\omega$, where $\omega$ is a $\mathbb{Z}_{+}$-linear combination of the $\alpha_{i}$. If $V_{\mu}^{0}$ is nonzero for a certain $\mu \neq \mu_{0}$ then a common eigenvector in $V_{\mu}^{0}$ for all $s_{i i}^{(r)}$ generates a proper submodule is $V$, which makes a contradiction.

Remark 3.4. The above proof implies that if $V$ is an irreducible highest weight representation of $\mathrm{Y}^{ \pm}(N)$ then the subspace (3.4) is one-dimensional and spanned by the highest weight vector $\xi$ of $V$.

Due to Theorem 3.3, to describe the finite-dimensional irreducible representations of $\mathrm{Y}^{ \pm}(N)$ it suffices to find necessary and sufficient conditions for an irreducible highest weight representation $V(\mu(u))$ to be finite-dimensional. Some necessary conditions are provided by the following proposition. We use notation $(2.35)$. 
Proposition 3.5. If the irreducible highest weight representation $V(\mu(u))$ of the algebra $\mathrm{Y}^{ \pm}(N)$ is finite-dimensional then the following relation holds:

$$
\mu_{1}(u) \rightarrow \mu_{2}(u) \rightarrow \cdots \rightarrow \mu_{n}(u)
$$

Proof. Let $J$ be the left ideal of $\mathrm{Y}^{ \pm}(N)$ generated by the coefficients of the series $s_{-i, j}(u)$, where $i, j=1, \ldots, n$. Denote by Norm $J$ the normalizer of $J$ :

$$
\text { Norm } J=\left\{y \in \mathrm{Y}^{ \pm}(N) \mid J y \subseteq J\right\}
$$

The annihilator $V(\mu(u))^{J}$ of $J$ in $V(\mu(u))$ has a natural structure of a Norm $J / J$ module. Note that the highest weight vector of $V(\mu(u))$ is contained in $V(\mu(u))^{J}$. Defining relations (1.17) show that the mapping

$$
t_{i j}^{(r)} \mapsto s_{i j}^{(r)} \quad \bmod J, \quad 1 \leq i, j \leq n
$$

defines an algebra homomorphism

$$
\mathrm{Y}(n) \rightarrow \operatorname{Norm} J / J
$$

By making use of this homomorphism we may regard $V(\mu(u))^{J}$ as a $\mathrm{Y}(n)$-module. The cyclic span of the vector $\xi$ is a finite-dimensional representation of $\mathrm{Y}(n)$ with the highest weight $\left(\mu_{1}(u), \ldots, \mu_{n}(u)\right)$. Applying Theorem 2.12 we complete the proof.

Given $\mu=\left(\mu_{1}, \ldots, \mu_{n}\right) \in \mathfrak{h}^{*}$ consider the irreducible $\mathfrak{g}(N)$-module with the highest weight $\mu$ with respect to the basis $\left\{F_{11}, \ldots, F_{n n}\right\}$ in $\mathfrak{h}^{*}$. It is finite-dimensional if and only if the following conditions are satisfied (see, e.g., [Di, Section 7.2]):

$$
\mu_{i+1}-\mu_{i} \in \mathbb{Z}_{+} \quad \text { for } \quad i=1, \ldots, n-1
$$

and

$$
\begin{array}{rlll}
-\mu_{1}-\mu_{2} & \in \mathbb{Z}_{+} & \text {for } & \mathfrak{o}(2 n), \\
-\mu_{1} \in \mathbb{Z}_{+} & \text {for } & \mathfrak{s p}(2 n), \\
-2 \mu_{1} \in \mathbb{Z}_{+} & \text {for } & \mathfrak{o}(2 n+1) .
\end{array}
$$

The homomorphism (1.23) allows us to extend it to a $\mathrm{Y}^{ \pm}(N)$-module. This module is obviously highest weight with

$$
\mu_{i}(u)=\frac{1+\left(\mu_{i} \pm 1 / 2\right) u^{-1}}{1 \pm 1 / 2 u^{-1}}, \quad i=1, \ldots, n
$$

and $\mu_{0}(u)=1$ if $N=2 n+1$. 
Using (1.31) we can equip any tensor product $L \otimes V$ of a $\mathrm{Y}(N)$-module $L$ and a $\mathrm{Y}^{ \pm}(N)$-module $V$ with a $\mathrm{Y}^{ \pm}(N)$-action by the rule

$$
y \cdot(\xi \otimes \eta):=\Delta(y)(\xi \otimes \eta), \quad y \in \mathrm{Y}^{ \pm}(N), \quad \xi \in L, \quad \eta \in V .
$$

In particular, we can construct representations of $\mathrm{Y}^{ \pm}(N)$ of the form $(0.3)$.

Decomposition (1.30) enables one to establish a correspondence between representations of the twisted Yangian $\mathrm{Y}^{ \pm}(N)$ and the special twisted Yangian $\mathrm{SY}^{ \pm}(N)$ analogous to the $A$ series case (see Section 2). Consider the similarity classes of finite-dimensional irreducible representations of $\mathrm{Y}^{ \pm}(N)$ : two representations belong to the same class if one can be obtained from the other by the composition with an automorphism (1.29). Since the subalgebra $\mathrm{SY}^{ \pm}(N)$ is stable with respect to all automorphisms of the form (1.29), any representation from such a class, restricted to the subalgebra $\mathrm{SY}^{ \pm}(N)$ gives the same representation of the latter. Moreover, all finite-dimensional irreducible representations of $\mathrm{SY}^{ \pm}(N)$ can be obtained in this way.

In the next three sections we consider separately the twisted Yangians corresponding to the series $\mathrm{C}, \mathrm{D}$, and $\mathrm{B}$, respectively. We obtain a description of finitedimensional irreducible representations for the algebras $\mathrm{Y}^{ \pm}(N)$ and $\mathrm{SY}^{ \pm}(N)$.

\section{Representations of $\mathrm{Y}^{-}(2 n)$}

As for the Yangian $\mathrm{Y}(N)$ the representations of the simplest twisted Yangian $\mathrm{Y}^{-}(2)$ play an important role in the general case.

Irreducible highest weight representations $V(\mu(u))$ of $\mathrm{Y}^{-}(2)$ are parameterized by formal series $\mu(u) \in 1+u^{-1} \mathbb{C}\left[\left[u^{-1}\right]\right]$. The following is an analogue of Proposition 2.5 for the algebra $\mathrm{Y}^{-}(2)$.

Proposition 4.1. If $\operatorname{dim} V(\mu(u))<\infty$ then there exists an even formal series $\psi(u) \in 1+u^{-2} \mathbb{C}\left[\left[u^{-2}\right]\right]$ such that $\psi(u) \mu(u)$ is a polynomial in $u^{-1}$.

Proof. The symmetry relation (1.18) implies that

$$
(2 u-1) s_{1,-1}(u)=(-2 u-1) s_{1,-1}(-u),
$$

and so, for any $r \geq 1$

$$
s_{1,-1}^{(2 r-1)}=2 s_{1,-1}^{(2 r)} .
$$

Denote by $\xi$ the highest weight vector of $V(\mu(u))$. Take the minimum nonnegative integer $k$ such that $s_{1,-1}^{(2 k+2)} \xi$ is a linear combination of the vectors $s_{1,-1}^{(2)} \xi, \ldots, s_{1,-1}^{(2 k)} \xi$; this $k$ exists because $V(\mu(u))$ is finite-dimensional. Let us prove that for any $r \geq$ $k+1$ we have

$$
s_{1,-1}^{(2 r)} \xi=a_{1}^{(2 r)} \xi_{1}+\cdots+a_{k}^{(2 r)} \xi_{k}
$$


for some complex coefficients $a_{i}^{(2 r)}$, where $\xi_{i}:=s_{1,-1}^{(2 i)} \xi, i=1, \ldots, k$. This is true for $r=k+1$ by our choice of $k$. Taking in (1.17) $i=k=l=1, j=-1$ and exchanging $u$ and $v$ we get

$$
\begin{aligned}
{\left[s_{11}(u), s_{1,-1}(v)\right]=} & \frac{2 u+1}{u^{2}-v^{2}} s_{1,-1}(v) s_{11}(u) \\
& -\frac{u+v+1}{u^{2}-v^{2}} s_{1,-1}(u) s_{11}(v)+\frac{1}{u+v} s_{1,-1}(u) s_{-1,-1}(v) .
\end{aligned}
$$

Take the coefficient at $u^{-3} v^{-2 p}$ in this relation. Using (4.1) and the fact that the series $s_{11}(u)+s_{-1,-1}(u)$ is even in $u$ (see (1.18)), we obtain

$$
\left[s_{11}^{(3)}, s_{1,-1}^{(2 p)}\right]=2 s_{1,-1}^{(2 p+2)}+s_{1,-1}^{(2 p)}\left(2 s_{11}^{(2)}+s_{11}^{(1)}\right)-s_{1,-1}^{(2)}\left(3 s_{11}^{(2 p)}+s_{-1,-1}^{(2 p)}\right) .
$$

Write $\mu(u)=1+\mu^{(1)} u^{-1}+\mu^{(2)} u^{-2}+\cdots$. Then applying both sides of (4.4) to $\xi$ we get

$$
2 s_{1,-1}^{(2 p+2)} \xi=s_{11}^{(3)} s_{1,-1}^{(2 p)} \xi-\left(\mu^{(3)}+2 \mu^{(2)}+\mu^{(1)}\right) s_{1,-1}^{(2 p)} \xi+\left(3 \mu^{(2 p)}+\mu_{-}^{(2 p)}\right) s_{1,-1}^{(2)} \xi,
$$

where $\mu_{-}^{(r)}$ is the eigenvalue of $s_{-1,-1}^{(r)}$ at $\xi$. In particular, for $p=1, \ldots, k-1$ we have

$$
s_{11}^{(3)} \xi_{p}=2 \xi_{p+1}+\left(\mu^{(3)}+2 \mu^{(2)}+\mu^{(1)}\right) \xi_{p}-\left(3 \mu^{(2 p)}+\mu_{-}^{(2 p)}\right) \xi_{1} .
$$

Applying (4.2) with $r=k+1$ gives

$$
\begin{aligned}
s_{11}^{(3)} \xi_{k} & =2\left(a_{1}^{(2 k+2)} \xi_{1}+\cdots+a_{k}^{(2 k+2)} \xi_{k}\right) \\
& +\left(\mu^{(3)}+2 \mu^{(2)}+\mu^{(1)}\right) \xi_{k}-\left(3 \mu^{(2 k)}+\mu_{-}^{(2 k)}\right) \xi_{1} .
\end{aligned}
$$

Now (4.2) follows from (4.5)-(4.7) by an induction on $r$.

Rewriting (4.2) in terms of generating series and using (4.1) we obtain

$$
s_{1,-1}(u) \xi=(2 u+1)\left(a_{1}(u) \xi_{1}+\cdots+a_{k}(u) \xi_{k}\right)
$$

where the $a_{i}(u)$ are even series in $u^{-1}: a_{i}(u)=u^{-2 i}+a_{i}^{(2 k+2)} u^{-2 k-2}+\cdots$.

Taking the coefficient at $u^{-3}$ in (4.3) and replacing $v$ with $u$ we get

$$
\begin{aligned}
s_{11}^{(3)} s_{1,-1}(u) & =s_{1,-1}(u)\left(s_{11}^{(3)}+2 s_{11}^{(2)}+s_{11}^{(1)}+2 u^{2}\right) \\
& -\left(s_{1,-1}^{(2)}+(u+1) s_{1,-1}^{(1)}\right) s_{11}(u)+\left(s_{1,-1}^{(2)}-u s_{1,-1}^{(1)}\right) s_{-1,-1}(u) .
\end{aligned}
$$

Apply both sides to the vector $\xi$. Then using (4.8) and the symmetry relation (1.18) we find that

$$
\begin{aligned}
\left(s_{11}^{(3)}-\mu^{(3)}-2 \mu^{(2)}-\right. & \left.\mu^{(1)}-2 u^{2}\right)\left(a_{1}(u) \xi_{1}+\cdots+a_{k}(u) \xi_{k}\right) \\
& =-\left(\frac{2 u+1}{2 u} \mu(u)+\frac{2 u-1}{2 u} \mu(-u)\right) \xi_{1} .
\end{aligned}
$$


Using (4.6) and (4.7) and taking the coefficient at $\xi_{p}, p=2, \ldots, k$ in this relation we get

$$
a_{p-1}(u)-u^{2} a_{p}(u)+\text { const } \cdot a_{k}(u)=0 .
$$

Therefore, for any $p=1, \ldots, k$ one has $a_{p}(u)=A_{p}(u) a_{k}(u)$ for a monic polynomial $A_{p}(u)$ of degree $k-p$ in $u^{2}$. Hence, taking the coefficient at $\xi_{1}$ in (4.9) we obtain that

$$
\left(\frac{2 u+1}{2 u} \mu(u)+\frac{2 u-1}{2 u} \mu(-u)\right)=B(u) a_{k}(u)
$$

for a polynomial $B(u)$ of degree $k$ in $u^{2}$.

Similarly, taking the coefficient at $u^{-2}$ in (4.3) we get

$$
s_{11}^{(2)} s_{1,-1}(u)=s_{1,-1}(u)\left(s_{11}^{(2)}+2 s_{11}^{(1)}+1\right)-s_{1,-1}^{(1)}\left(s_{11}(u)-s_{-1,-1}(u)\right) .
$$

Applying both sides to $\xi$, using (4.8) and the symmetry relation (1.18) we obtain

$$
\left(s_{11}^{(2)}-\mu^{(2)}-2 \mu^{(1)}-1\right)\left(a_{1}(u) \xi_{1}+\cdots+a_{k}(u) \xi_{k}\right)=-u^{-1}(\mu(u)-\mu(-u)) \xi_{1} .
$$

In particular, $s_{11}^{(2)} \xi_{p}$ is a linear combination of $\xi_{p}$ and $\xi_{1}$. Finally, taking the coefficient at $\xi_{1}$ in $(4.12)$ we find that $u^{-1}(\mu(u)-\mu(-u))=C(u) a_{k}(u)$ for a polynomial $C(u)$ of degree $k-1$ in $u^{2}$. Together with (4.10) this proves that the desired series is $\psi(u)=\left(u^{2 k} a_{k}(u)\right)^{-1}$.

We shall consider (2.16) as a representation of $\mathrm{Y}^{-}(2)$ obtained by restriction. Equivalently, using (3.11) one may regard this representation as the tensor product of $\mathrm{Y}(2)$-module (2.16) with the trivial $\mathrm{Y}^{-}(2)$-module.

Let $\mu(u)$ be a polynomial in $u^{-1}$ of degree $\leq 2 k$. Consider an expansion

$$
\mu(u)=\left(1-\gamma_{1} u^{-1}\right) \cdots\left(1-\gamma_{2 k} u^{-1}\right)
$$

Re-enumerating the numbers $\gamma_{i}$ if necessary, we may assume that the following condition is satisfied (cf. (2.19)):

for every $i=1, \ldots, k$ we have:

if the set $\left\{\gamma_{p}+\gamma_{q} \mid 2 i-1 \leq p<q \leq 2 k\right\} \cap \mathbb{Z}_{+}$is not empty

then $\gamma_{2 i-1}+\gamma_{2 i}$ is its minimal element.

The following is an analogue of Proposition 2.6.

Proposition 4.2. Let $V(\mu(u))$ be an irreducible highest weight representation of $\mathrm{Y}^{-}(2)$ where $\mu(u)$ is a polynomial of degree $\leq 2 k$ in $u^{-1}$. Suppose that decomposition (4.13) is given and condition (4.14) is satisfied. Then $V(\mu(u))$ is isomorphic to the tensor product

$$
L\left(\gamma_{1},-\gamma_{2}\right) \otimes \cdots \otimes L\left(\gamma_{2 k-1},-\gamma_{2 k}\right)
$$


regarded as $\mathrm{Y}^{-}(2)$-module.

Proof. Denote representation (4.15) by $V$. Let $\xi_{i}$ be the highest weight vector of $L\left(\gamma_{2 i-1},-\gamma_{2 i}\right)$. We obtain from $(2.23)$ that the vector $\xi:=\xi_{1} \otimes \cdots \otimes \xi_{k}$ satisfies the relations

$$
\begin{aligned}
t_{-1,1}(u) \xi & =0 \\
t_{11}(u) \xi & =\left(1-\gamma_{2} u^{-1}\right) \cdots\left(1-\gamma_{2 k} u^{-1}\right) \xi \\
t_{-1,-1}(u) \xi_{i} & =\left(1+\gamma_{1} u^{-1}\right) \cdots\left(1+\gamma_{2 k-1} u^{-1}\right) \xi .
\end{aligned}
$$

Hence, by (1.15)

$$
s_{11}(u) \xi=\mu(u) \xi \quad \text { and } \quad s_{-1,1}(u) \xi=0 .
$$

Thus, the cyclic $\mathrm{Y}^{-}(2)$-span of the vector $\xi$ in $V$ is a highest weight module with the highest weight $\mu(u)$. Therefore, to prove Proposition 4.2 it suffices to show that the representation $V$ of $\mathrm{Y}^{-}(2)$ is irreducible. We use induction on $k$.

For $k=1$ this is obvious, because the restriction of $L\left(\gamma_{2 i-1},-\gamma_{2 i}\right)$ to $\mathfrak{s p}(2)$ is irreducible and so its extension to $\mathrm{Y}^{-}(2)$ is.

Suppose now that $k>1$. If $\widetilde{V}$ is a nonzero submodule of $V$ then $\widetilde{V}$ contains a nonzero vector $\eta$ such that $s_{-1,1}(u) \eta=0$. Write $\eta$ in the form

$$
\eta=\sum_{r=0}^{p}\left(E_{1,-1}\right)^{r} \xi_{1} \otimes \eta_{r}
$$

for some $p \geq 0$, where $\eta_{r} \in L\left(\gamma_{3},-\gamma_{4}\right) \otimes \cdots \otimes L\left(\gamma_{2 k-1},-\gamma_{2 k}\right)$; and if $\gamma_{1}+\gamma_{2} \in \mathbb{Z}_{+}$ then $p \leq \gamma_{1}+\gamma_{2}$. We may assume that $\eta_{p} \neq 0$. By (1.31) we have

$$
\begin{aligned}
& s_{-1,1}(u)\left(\left(E_{1,-1}\right)^{r} \xi_{1} \otimes \eta_{r}\right)=-t_{-1,-1}(u) t_{-1,1}(-u)\left(E_{1,-1}\right)^{r} \xi_{1} \otimes s_{-1,-1}(u) \eta_{r} \\
& +t_{-1,-1}(u) t_{-1,-1}(-u)\left(E_{1,-1}\right)^{r} \xi_{1} \otimes s_{-1,1}(u) \eta_{r} \\
& -t_{-1,1}(u) t_{-1,1}(-u)\left(E_{1,-1}\right)^{r} \xi_{1} \otimes s_{1,-1}(u) \eta_{r} \\
& +t_{-1,1}(u) t_{-1,-1}(-u)\left(E_{1,-1}\right)^{r} \xi_{1} \otimes s_{1,1}(u) \eta_{r} \text {. }
\end{aligned}
$$

Rewriting (2.25) and (2.26) we obtain

$$
t_{-1,-1}(u)\left(E_{1,-1}\right)^{r} \xi_{1}=\left(1+\left(\gamma_{1}-r\right) u^{-1}\right)\left(E_{1,-1}\right)^{r} \xi_{1}
$$

and

$$
t_{-1,1}(u)\left(E_{1,-1}\right)^{r} \xi_{1}=u^{-1} r\left(\gamma_{1}+\gamma_{2}-r+1\right)\left(E_{1,-1}\right)^{r-1} \xi_{1} .
$$

Taking the coefficient at $\left(E_{1,-1}\right)^{p} \xi_{1}$ in the relation $s_{-1,1}(u) \eta=0$ gives

$$
\left(1-\left(\gamma_{1}-p\right) u^{-2}\right) s_{-1,1}(u) \eta_{p}=0
$$


So, $s_{-1,1}(u) \eta_{p}=0$. By the induction hypotheses, the representation $L\left(\gamma_{3},-\gamma_{4}\right) \otimes$ $\cdots \otimes L\left(\gamma_{2 k-1},-\gamma_{2 k}\right)$ of $\mathrm{Y}^{-}(2)$ is irreducible, and hence

$$
\eta_{p}=\operatorname{const} \cdot \xi_{2} \otimes \cdots \otimes \xi_{k}
$$

where 'const' is a nonzero constant; see Remark 3.4.

Suppose now that $p \geq 1$ and take the coefficient at $\left(E_{1,-1}\right)^{p-1} \xi_{1}$ in the relation $s_{-1,1}(u) \eta=0$. Using (4.19)-(4.21) we obtain

$$
\begin{aligned}
\left(1-\left(\gamma_{1}-\right.\right. & \left.p+1)^{2} u^{-2}\right) s_{-1,1}(u) \eta_{p-1} \\
& +u^{-1} p\left(\gamma_{1}+\gamma_{2}-p+1\right)\left(1+\left(\gamma_{1}-p+1\right) u^{-1}\right) s_{-1,-1}(u) \eta_{p} \\
& +u^{-1} p\left(\gamma_{1}+\gamma_{2}-p+1\right)\left(1-\left(\gamma_{1}-p\right) u^{-1}\right) s_{11}(u) \eta_{p}=0
\end{aligned}
$$

The symmetry relation (1.18) gives

$$
s_{-1,-1}(u)=\frac{2 u+1}{2 u} s_{11}(-u)-\frac{1}{2 u} s_{11}(u)
$$

and

$$
s_{-1,1}^{(2 r-1)}=2 s_{-1,1}^{(2 r)}, \quad r \geq 1
$$

Using (4.24) and multiplying (4.23) by $u^{2 k} /(2 u+1)$ we bring it to the form

$$
\begin{aligned}
& \left(u^{2}-\left(\gamma_{1}-p+1\right)^{2}\right) u^{2 k-2} \frac{s_{-1,1}(u)}{2 u+1} \eta_{p-1}+p\left(\gamma_{1}+\gamma_{2}-p+1\right) \\
& \times \frac{1}{2 u}\left(\left(u+\gamma_{1}-p+1\right) u^{2 k-2} s_{11}(-u)+\left(u-\gamma_{1}+p-1\right) u^{2 k-2} s_{11}(u)\right) \eta_{p}=0 .
\end{aligned}
$$

Due to (2.18) and (1.15) the elements $s_{i j}^{(r)}$ with $r>2 k-2$ act as zero operators in $L\left(\gamma_{3},-\gamma_{4}\right) \otimes \cdots \otimes L\left(\gamma_{2 k-1},-\gamma_{2 k}\right)$. Hence, the operators $u^{2 k-2} s_{i j}(u)$ are polynomials in $u$, as well as $u^{2 k-2} s_{-1,1}(u) /(2 u+1)$ is, because of (4.25). Further, by (4.22) and $(4.16)$

$$
u^{2 k-2} s_{11}(u) \eta_{p}=\left(u-\gamma_{3}\right) \cdots\left(u-\gamma_{2 k}\right) \eta_{p} .
$$

Putting $u=\gamma_{1}-p+1$ in $(4.26)$ we get

$$
p\left(\gamma_{1}+\gamma_{2}-p+1\right)\left(\gamma_{1}+\gamma_{3}-p+1\right) \cdots\left(\gamma_{1}+\gamma_{2 k}-p+1\right)=0
$$

However, this is impossible because of condition (4.14). Therefore, $p$ has to be equal to 0 and so, $\eta$ is a multiple of $\xi$.

To complete the proof we have to show that the submodule of $V$ generated by $\xi$ coincides with $V$.

The representation of $\mathrm{Y}(2)$ dual to $(4.15)$ is isomorphic to

$$
L^{\prime}\left(-\gamma_{1}, \gamma_{2}\right) \otimes \cdots \otimes L^{\prime}\left(-\gamma_{2 k-1}, \gamma_{2 k}\right)
$$


see (2.31). On the other hand, using the antiautomorphism (1.26) we can equip the vector space $V^{*}$ dual to $V$ with an action of $\mathrm{Y}^{-}(2)$ :

$$
(y \cdot f)(v)=f(\sigma(y) v), \quad y \in \mathrm{Y}^{-}(2), \quad f \in V^{*}, \quad v \in V .
$$

Since the antiautomorphism (1.26) is a restriction of (1.7), the representation $V^{*}$ is isomorphic to the restriction of (4.27) to $\mathrm{Y}^{-}(2)$.

Similarly to the case of representation $V$, any nonzero submodule of $V^{*}$ contains a nonzero vector $\eta^{\prime}$ such that $s_{1,-1}(u) \eta^{\prime}=0$. Modifying the previous argument for lowest weight representations of $\mathrm{Y}^{-}(2)$ we prove that $\eta^{\prime}$ coincides (up to a nonzero factor) with the vector $\xi^{\prime}=\xi_{1}^{\prime} \otimes \cdots \otimes \xi_{k}^{\prime}$, where $\xi_{i}^{\prime}$ is the lowest weight vector of $L^{\prime}\left(-\gamma_{2 i-1}, \gamma_{2 i}\right)$. Now, if the submodule in $V$ generated by $\xi$ is proper, then its annihilator in $V^{*}$ is a nonzero submodule which does not contain $\xi^{\prime}$. Contradiction.

We are now able to give necessary and sufficient conditions for $V(\mu(u))$ to be finite-dimensional (cf. Proposition 2.7).

Proposition 4.3. Let $V(\mu(u))$ be an irreducible representation of $\mathrm{Y}^{-}(2)$ with the highest weight $\mu(u)$. Then $V(\mu(u))$ is finite-dimensional if and only if there exists a formal series $\psi(u) \in 1+u^{-2} \mathbb{C}\left[\left[u^{-2}\right]\right]$ such that $\psi(u) \mu(u)$ is a polynomial in $u^{-1}$ with a decomposition

$$
\psi(u) \mu(u)=\left(1-\gamma_{1} u^{-1}\right) \cdots\left(1-\gamma_{2 k} u^{-1}\right),
$$

and $\gamma_{2 i-1}+\gamma_{2 i} \in \mathbb{Z}_{+}, i=1, \ldots, k$ for a certain re-enumeration of the $\gamma_{i}$.

Proof. Let $\operatorname{dim} V(\mu(u))<\infty$. By Proposition 4.1, taking the composition of our representation with an automorphism of the form (1.29) we may assume that (4.28) is satisfied for a certain series $\psi(u)$. Re-enumerate the $\gamma_{i}$ in such a way that condition (4.14) be satisfied. Now use Proposition 4.2. Since representation (4.15) is finite-dimensional this gives the relations $\gamma_{2 i-1}+\gamma_{2 i} \in \mathbb{Z}_{+}$for all $i=1, \ldots, k$.

Conversely, let (4.28) hold with $\gamma_{2 i-1}+\gamma_{2 i} \in \mathbb{Z}_{+}, i=1, \ldots, k$. Then representation (4.15) is finite-dimensional and the composition of $V(\mu(u))$ with the automorphism (1.29) corresponding to the series $\psi(u)$ is isomorphic to the irreducible quotient of the $\mathrm{Y}^{-}(2)$-cyclic span in $V$ of the vector $\xi$; see (4.17). Therefore, $\operatorname{dim} V(\mu(u))<\infty$.

Proposition 4.3 can be reformulated with the use of monic polynomials in $u$ as follows (cf. Theorem 2.8).

Theorem 4.4. The irreducible highest weight representation $V(\mu(u))$ of $\mathrm{Y}^{-}(2)$ is finite-dimensional if and only if there exists a monic polynomial $P(u) \in \mathbb{C}[u]$ such that $P(u)=P(-u+1)$ and

$$
\frac{\mu(-u)}{\mu(u)}=\frac{P(u+1)}{P(u)} .
$$


In this case $P(u)$ is unique.

Proof. If $\operatorname{dim} V(\mu(u))<\infty$ then by Proposition 4.3 relation (4.28) holds for a certain even series $\psi(u)$ and $\gamma_{2 i-1}+\gamma_{2 i} \in \mathbb{Z}_{+}$for all $i$. Set

$$
\begin{aligned}
\sigma(u) & =\left(1+\gamma_{1} u^{-1}\right)\left(1+\gamma_{3} u^{-1}\right) \cdots\left(1+\gamma_{2 k-1} u^{-1}\right), \\
\tau(u) & =\left(1-\gamma_{2} u^{-1}\right)\left(1-\gamma_{4} u^{-1}\right) \cdots\left(1-\gamma_{2 k} u^{-1}\right) .
\end{aligned}
$$

Then there exists a monic polynomial $Q(u)$ in $u$ such that

$$
\frac{\sigma(u)}{\tau(u)}=\frac{Q(u+1)}{Q(u)}
$$

see the proof of Theorem 2.8. Define $P(u)$ by

$$
P(u)=Q(u) Q(-u+1)(-1)^{\operatorname{deg} Q} .
$$

Then $P(u)$ is monic and $P(u)=P(-u+1)$. Moreover,

$$
\frac{\tau(-u)}{\sigma(-u)}=\frac{Q(-u)}{Q(-u+1)} \text {. }
$$

Therefore, since $\psi(u) \mu(u)=\sigma(-u) \tau(u)$, relation (4.29) holds due to (4.31) and (4.33).

Conversely, let $(4.29)$ be satisfied for a monic polynomial $P(u)$ such that $P(u)=$ $P(-u+1)$. Then it has an even degree and the multiset of its roots is invariant with respect to the transformation $u \mapsto-u+1$ (the symmetry in $\mathbb{C}$ with the center at $1 / 2)$. Therefore, we may write the roots in the form $\left\{-\delta_{1}, \ldots,-\delta_{s}, \delta_{1}+1, \ldots, \delta_{s}+1\right\}$ for a certain $s$. Set

$$
\mu^{\prime}(u)=\left(1-\left(\delta_{1}+1\right) u^{-1}\right) \cdots\left(1-\left(\delta_{s}+1\right) u^{-1}\right)\left(1+\delta_{1} u^{-1}\right) \cdots\left(1+\delta_{s} u^{-1}\right) .
$$

By Proposition 4.3 the representation $V\left(\mu^{\prime}(u)\right)$ is finite-dimensional. Moreover, the polynomial $\mu^{\prime}(u)$ satisfies (4.29) and so, the ratio $\mu(u) / \mu^{\prime}(u)$ is an even formal series in $u^{-1}$. This proves that $V(\mu(u))$ is also finite-dimensional.

The uniqueness of $P(u)$ is established in the proof of Theorem 2.8.

The following notation is motivated by Theorem 4.4 (cf. (2.35)). For two formal series $\mu(u)$ and $\nu(u)$ in $u^{-1}$ we shall write

$$
\mu(u) \Rightarrow \nu(u)
$$

if there exists a monic polynomial $P(u)$ in $u$ such that $P(u)=P(-u+1)$ and

$$
\frac{\mu(u)}{\nu(u)}=\frac{P(u+1)}{P(u)} .
$$

Obviously, this is only possible if $\mu(u)$ and $\nu(u)$ satisfy the relation $\mu(u) \mu(-u)=$ $\nu(u) \nu(-u)$.

Proposition 4.2 and Theorem 4.4 imply the following parameterization of representations of the special twisted Yangian $\mathrm{SY}^{-}$(2) (cf. Corollary 2.9). 
Corollary 4.5. There is a one-to-one correspondence between finite-dimensional irreducible representations of the special twisted Yangian $\mathrm{SY}^{-}(2)$ and the monic polynomials $P(u)$ in $u$ such that $P(u)=P(-u+1)$. Every such representation is isomorphic to a representation of the form (4.15).

Remark 4.6. Tensoring $V(\mu(u))$ by factors of the form $L(\gamma, \gamma)$ is equivalent to multiplying the highest weight $\mu(u)$ by $1-\gamma^{2} u^{-2}$. However, the representations $V(\mu(u))$ and $V\left(\left(1-\gamma^{2} u^{-2}\right) \mu(u)\right)$ of $\mathrm{Y}^{-}(2)$ belong to the same similarity class. Therefore, we might add to Corollary 4.5 the condition that in (4.15) all $\gamma_{2 i-1}+\gamma_{2 i}$ are positive integers.

The following corollary provides a criterion of irreducibility for the representations of $\mathrm{Y}^{-}(2)$ (or $\mathrm{SY}^{-}(2)$ ) of the form (4.15) (cf. Corollary 2.11). We formulate it in terms of the strings $S(\alpha, \beta)$ introduced in Section 2. Note that two strings $S(\alpha,-\beta)$ and $S(\beta,-\alpha)$ are symmetric to each other with respect to the center $-1 / 2$ in $\mathbb{C}$. Therefore, $S\left(\alpha_{1},-\beta_{1}\right)$ and $S\left(\alpha_{2},-\beta_{2}\right)$ are in general position whenever $S\left(\beta_{1},-\alpha_{1}\right)$ and $S\left(\beta_{2},-\alpha_{2}\right)$ are.

Corollary 4.7. Consider an irreducible finite-dimensional representation of $\mathrm{Y}(2)$ of the form

$$
V=L\left(\gamma_{1},-\gamma_{2}\right) \otimes \cdots \otimes L\left(\gamma_{2 k-1},-\gamma_{2 k}\right)
$$

Then its restriction to $\mathrm{Y}^{-}(2)$ is irreducible if and only if for each pair $i<j$ the strings $S\left(\gamma_{2 i},-\gamma_{2 i-1}\right)$ and $S\left(\gamma_{2 j-1},-\gamma_{2 j}\right)$ are in general position.

Proof. By Corollary 2.11, the strings $S\left(\gamma_{2 i-1},-\gamma_{2 i}\right), i=1, \ldots, k$ are pairwise in general position. Permuting the tensor factors in (4.35) if necessary, we may assume that $\gamma_{1}+\gamma_{2} \leq \cdots \leq \gamma_{2 k-1}+\gamma_{2 k}$. Suppose that the strings $S\left(\gamma_{2 i},-\gamma_{2 i-1}\right)$ and $S\left(\gamma_{2 j-1},-\gamma_{2 j}\right)$ are in general position for all $i<j$. Then one easily checks that (4.14) is satisfied and so, by Proposition 4.2 representation (4.35) of $\mathrm{Y}^{-}(2)$ is irreducible.

Conversely, let $V$ be irreducible as a $\mathrm{Y}^{-}(2)$-module. Consider the module $V^{\prime}$ obtained by replacement of some of the tensor factors $L\left(\gamma_{2 i-1},-\gamma_{2 i}\right)$ respectively by $L\left(\gamma_{2 i},-\gamma_{2 i-1}\right)$. Then $V^{\prime}$ is isomorphic to $V$. Indeed, $\operatorname{dim} V=\operatorname{dim} V^{\prime}$ and one easily checks using (1.15) that $V$ is isomorphic to the irreducible quotient of the $\mathrm{Y}^{-}(2)$-submodule in $V^{\prime}$ generated by the tensor product of the highest weight vectors of the factors. So, $V^{\prime}$ is irreducible as a $\mathrm{Y}^{-}(2)$-module and hence, as a $\mathrm{Y}(2)$-module. By Corollary 2.11 the corresponding strings are pairwise in general position.

The following theorem together with Theorem 3.3 gives a description of finitedimensional irreducible representations of $\mathrm{Y}^{-}(2 n)$ (cf. Theorem 2.12). We use notation (2.35) and (4.34). 
Theorem 4.8. The irreducible highest weight representation $V(\mu(u)), \mu(u)=$ $\left(\mu_{1}(u), \ldots, \mu_{n}(u)\right)$ of $\mathrm{Y}^{-}(2 n)$ is finite-dimensional if and only if the following relation holds

$$
\mu_{1}(-u) \Rightarrow \mu_{1}(u) \rightarrow \mu_{2}(u) \rightarrow \cdots \rightarrow \mu_{n}(u) .
$$

Proof. Suppose that $\operatorname{dim} V(\mu(u))<\infty$. By Proposition 3.5 we have

$$
\mu_{1}(u) \rightarrow \mu_{2}(u) \rightarrow \cdots \rightarrow \mu_{n}(u)
$$

The subalgebra in $\mathrm{Y}^{-}(2 n)$ generated by the coefficients of the series $s_{i j}(u)$ with $i, j=-1,1$ is isomorphic to $\mathrm{Y}^{-}(2)$. The cyclic span of the highest weight vector of $V(\mu(u))$ with respect to this subalgebra is a representation with the highest weight $\mu_{1}(u)$. Its irreducible quotient is finite-dimensional and so, by Theorem 4.4 we have $\mu_{1}(-u) \Rightarrow \mu_{1}(u)$.

Conversely, let (4.36) hold. Then

$$
\frac{\mu_{1}(-u)}{\mu_{1}(u)}=\frac{P_{1}(u+1)}{P_{1}(u)}
$$

and

$$
\frac{\mu_{i-1}(u)}{\mu_{i}(u)}=\frac{P_{i}(u+1)}{P_{i}(u)}
$$

for certain polynomials $P_{i}(u)$ of the form

$$
P_{i}(u)=\left(u+\delta_{1}^{(i)}\right) \cdots\left(u+\delta_{s_{i}}^{(i)}\right), \quad i=2, \ldots, n
$$

and

$$
P_{1}(u)=\left(u+\delta_{1}^{(1)}\right) \cdots\left(u+\delta_{s_{1}}^{(1)}\right)\left(u-\delta_{1}^{(1)}-1\right) \cdots\left(u-\delta_{s_{1}}^{(1)}-1\right)
$$

see the proof of Theorem 4.4 .

Consider the irreducible highest weight representation $L(\lambda(u))$ with $\lambda(u)=$ $\left(\lambda_{-n}(u), \ldots, \lambda_{n}(u)\right)$ of the Yangian $\mathrm{Y}(2 n)$ where for $i=1, \ldots, n$

$$
\begin{aligned}
\lambda_{i}(u) & =\prod_{a=1}^{i}\left(1+\delta_{1}^{(a)} u^{-1}\right) \cdots\left(1+\delta_{s_{a}}^{(a)} u^{-1}\right) \\
& \times \prod_{a=i+1}^{n}\left(1+\left(\delta_{1}^{(a)}+1\right) u^{-1}\right) \cdots\left(1+\left(\delta_{s_{a}}^{(a)}+1\right) u^{-1}\right)
\end{aligned}
$$

and

$$
\lambda_{-i}(u)=\prod_{a=1}^{n}\left(1+\left(\delta_{1}^{(a)}+1\right) u^{-1}\right) \cdots\left(1+\left(\delta_{s_{a}}^{(a)}+1\right) u^{-1}\right) .
$$


Then $L(\lambda(u))$ is finite-dimensional by Theorem 2.12. One easily derives from relations (1.1) and (1.15) that the cyclic $\mathrm{Y}^{-}(2 n)$-span of its highest weight vector is a representation with the highest weight $\mu^{\prime}(u)=\left(\mu_{1}^{\prime}(u), \ldots, \mu_{n}^{\prime}(u)\right)$ where $\mu_{i}^{\prime}(u)=$ $\lambda_{i}(u) \lambda_{-i}(-u)$. So, the representation $V\left(\mu^{\prime}(u)\right)$ of $\mathrm{Y}^{-}(2 n)$ is finite-dimensional. However, the polynomials $\mu_{i}^{\prime}(u)$ satisfy (4.38) and (4.39). Hence, there exists an automorphism of $\mathrm{Y}^{-}(2 n)$ of the form (1.29) such that its composition with the representation $V\left(\mu^{\prime}(u)\right)$ is isomorphic to $V(\mu(u))$ which completes the proof.

Theorem 4.8 implies the following parameterization of representations of the special twisted Yangian $\mathrm{SY}^{-}(2 n)$ (cf. Corollary 2.13).

Corollary 4.9. There is a one-to-one correspondence between finite-dimensional irreducible representations of the special twisted Yangian $\mathrm{SY}^{-}(2 n)$ and the families $\left\{P_{1}(u), \ldots, P_{n}(u)\right\}$ of monic polynomials in $u$ with $P_{1}(u)=P_{1}(-u+1)$. Every such representation is isomorphic to a subquotient of a representation of the form $L(\lambda(u))$.

\section{Representations of $\mathrm{Y}^{+}(2 n)$}

The results for the $D$ series are very similar to the previous case. However, some differences take place for $n=1$ since the twisted Yangian $\mathrm{Y}^{+}(2)$ corresponds to the abelian Lie algebra $\mathfrak{o}(2)$. In particular, contrary to the case of $\mathrm{Y}^{-}(2)$, it is not true that every finite-dimensional irreducible representations of $\mathrm{Y}^{+}(2)$ is obtained by restriction of a representation of $\mathrm{Y}(2)$. Also, the step from $\mathrm{Y}^{+}(2)$ to $\mathrm{Y}^{+}(2 n)$, $n \geq 2$ requires an extra care.

We omit the most of the proofs in this section which are parallel to the case of $\mathrm{Y}^{-}(2)$ but provide all necessary additional arguments.

Irreducible highest weight representations $V(\mu(u))$ of $\mathrm{Y}^{+}(2)$ are parameterized by formal series $\mu(u) \in 1+u^{-1} \mathbb{C}\left[\left[u^{-1}\right]\right]$.

Proposition 5.1. If $\operatorname{dim} V(\mu(u))<\infty$ then there exists an even formal series $\psi(u) \in 1+u^{-2} \mathbb{C}\left[\left[u^{-2}\right]\right]$ such that $\left(1+1 / 2 u^{-1}\right) \psi(u) \mu(u)$ is a polynomial in $u^{-1}$.

Proof. We repeat the argument of the proof of Proposition 4.1 almost word by word and only point out a few differences. First, here the symmetry relation (1.18) gives

$$
s_{1,-1}(u)=s_{1,-1}(-u),
$$

and so, for any $r \geq 1$

$$
s_{1,-1}^{(2 r-1)}=0
$$

Relation (4.3) is replaced by

$$
\begin{aligned}
{\left[s_{11}(u), s_{1,-1}(v)\right] } & =\frac{2 u+1}{u^{2}-v^{2}} s_{1,-1}(v) s_{11}(u) \\
& -\frac{u+v+1}{u^{2}-v^{2}} s_{1,-1}(u) s_{11}(v)-\frac{1}{u+v} s_{1,-1}(u) s_{-1,-1}(v) .
\end{aligned}
$$


Instead of (4.8) we obtain here

$$
s_{1,-1}(u) \xi=a_{1}(u) \xi_{1}+\cdots+a_{k}(u) \xi_{k}
$$

The counterpart of relation (4.10) has exactly the same form. However, for the remaining part of the proof instead of (4.11) we need to consider the following formula obtained from (5.1) by taking the coefficient at $u^{-4}$ :

$$
\begin{aligned}
s_{11}^{(4)} s_{1,-1}(u) & =s_{1,-1}(u)\left(s_{11}^{(4)}+2 s_{11}^{(3)}+s_{11}^{(2)}+2 s_{11}^{(1)} u^{2}+u^{2}\right) \\
& -s_{1,-1}^{(2)}\left((u+1) s_{11}(u)-u s_{-1,-1}(u)\right) .
\end{aligned}
$$

Applying both sides to $\xi$ and using the symmetry relation (1.18) we find that

$$
\left(u+\frac{1}{2}\right) \mu(u)-\left(u-\frac{1}{2}\right) \mu(-u)=C(u) a_{k}(u)
$$

for a polynomial $C(u)$ of degree $k$ in $u^{2}$. Together with (4.10) this proves that for $\psi(u)=\left(u^{2 k} a_{k}(u)\right)^{-1}$ the series $\left(1+1 / 2 u^{-1}\right) \psi(u) \mu(u)$ is a polynomial in $u^{-1}$ of degree $\leq 2 k+1$.

Note that the irreducible representation of $\mathrm{Y}^{+}(2)$ with the highest weight $\mu(u)=$ $\left(1+\gamma u^{-1}\right)\left(1+1 / 2 u^{-1}\right)^{-1}$, where $\gamma \in \mathbb{C}$ is one-dimensional. It is obtained by extending the one-dimensional representation of the abelian Lie algebra $\mathfrak{o}(2)$ given by $F_{11} \xi=(\gamma-1 / 2) \xi$, where $\xi$ is the basis vector; see (3.10). We shall denote this representation of $\mathrm{Y}^{+}(2)$ by $V(\gamma)$.

Let $\left(1+1 / 2 u^{-1}\right) \mu(u)$ be polynomial in $u^{-1}$ of degree $\leq 2 k+1$. Consider an expansion

$$
\mu(u)=\left(1-\gamma_{1} u^{-1}\right) \cdots\left(1-\gamma_{2 k+1} u^{-1}\right)\left(1+\frac{1}{2} u^{-1}\right)^{-1}
$$

Re-enumerating the numbers $\gamma_{i}$ if necessary, we may assume that the following condition is satisfied (cf. (4.14)):

for every $i=1, \ldots, k$ we have:

if the set $\left\{\gamma_{p}+\gamma_{q} \mid 2 i-1 \leq p<q \leq 2 k+1\right\} \cap \mathbb{Z}_{+}$is not empty then $\gamma_{2 i-1}+\gamma_{2 i}$ is its minimal element.

In the following proposition we use tensor products of representations constructed in accordance with (3.11). As before, we denote by $L(\alpha, \beta)$ a highest weight representation of the Lie algebra $\mathfrak{g l}(2)$ which is also regarded as a $\mathrm{Y}(2)$-module. 
Proposition 5.2. Let $V(\mu(u))$ be an irreducible highest weight representation of $\mathrm{Y}^{+}(2)$ where $\left(1+1 / 2 u^{-1}\right) \mu(u)$ is a polynomial of degree $\leq 2 k+1$ in $u^{-1}$. Suppose that decomposition (5.2) is given and condition (5.3) is satisfied. Then $V(\mu(u))$ is isomorphic to the tensor product

$$
L\left(\gamma_{1},-\gamma_{2}\right) \otimes \cdots \otimes L\left(\gamma_{2 k-1},-\gamma_{2 k}\right) \otimes V\left(-\gamma_{2 k+1}\right)
$$

Proof. Denote representation (5.4) by $V$. Let $\xi_{i}$ be the highest weight vector of $L\left(\gamma_{2 i-1},-\gamma_{2 i}\right)$ and $\xi_{k+1}$ be the basis vector of the one-dimensional representation $V\left(-\gamma_{2 k+1}\right)$.

Using (2.23) and (1.31) we find that the vector $\xi:=\xi_{1} \otimes \cdots \otimes \xi_{k+1}$ satisfies the relations

$$
s_{11}(u) \xi=\mu(u) \xi \quad \text { and } \quad s_{-1,1}(u) \xi=0 .
$$

Thus, the cyclic $\mathrm{Y}^{+}(2)$-span of the vector $\xi$ in $V$ is a highest weight module with the highest weight $\mu(u)$. So, it suffices to show that the representation $V$ is irreducible. We use induction on $k$.

For $k=0$ this is obvious. Suppose that $k \geq 1$. Proceeding exactly as in the proof of Proposition 4.2 we obtain that if the vector $\eta$ is given by (4.18) with

$$
\eta_{r} \in L\left(\gamma_{3},-\gamma_{4}\right) \otimes \cdots \otimes L\left(\gamma_{2 k-1},-\gamma_{2 k}\right) \otimes V\left(-\gamma_{2 k+1}\right)
$$

then

$$
\eta_{p}=\text { const } \cdot \xi_{2} \otimes \cdots \otimes \xi_{k+1},
$$

where 'const' is a nonzero constant. Further, instead of (4.26) we get here

$$
\begin{aligned}
& \left(u^{2}-\left(\gamma_{1}-p+1\right)^{2}\right) u^{2 k-1} s_{-1,1}(u) \eta_{p-1}+p\left(\gamma_{1}+\gamma_{2}-p+1\right) u^{2 k-1} \times \\
& \left(\frac{2 u+1}{2 u}\left(u-\gamma_{1}+p-1\right) s_{11}(u)-\frac{2 u-1}{2 u}\left(u+\gamma_{1}-p+1\right) s_{11}(-u)\right) \eta_{p}=0 .
\end{aligned}
$$

The elements $s_{-1,1}^{(r)}$ and $s_{1,-1}^{(r)}$ act trivially in $V\left(-\gamma_{2 k+1}\right)$. Hence, by (1.31) and (3.11) we have

$$
\begin{aligned}
s_{-1,1}(u)\left(\zeta \otimes \xi_{k+1}\right) & =t_{-1,1}(u) t_{-1,-1}(-u) \zeta \otimes s_{11}(u) \xi_{k+1} \\
& +t_{-1,-1}(u) t_{-1,1}(-u) \zeta \otimes s_{-1,-1}(u) \xi_{k+1}
\end{aligned}
$$

where $\zeta \in L\left(\gamma_{3},-\gamma_{4}\right) \otimes \cdots \otimes L\left(\gamma_{2 k-1},-\gamma_{2 k}\right)$. Using the defining relations (1.1) and the symmetry relation (1.18) we may rewrite this as follows

$$
\begin{aligned}
s_{-1,1}(u)\left(\zeta \otimes \xi_{k+1}\right) & =t_{-1,-1}(-u) t_{-1,1}(u) \zeta \otimes \frac{2 u+1}{2 u} s_{11}(u) \xi_{k+1} \\
& +t_{-1,-1}(u) t_{-1,1}(-u) \zeta \otimes \frac{2 u-1}{2 u} s_{11}(-u) \xi_{k+1} .
\end{aligned}
$$


Now, the definition of $V(\gamma)$ and (2.18) imply that $u^{2 k-1} s_{-1,1}(u) \eta_{p-1}$ is a polynomial in $u$. Further, by (5.5) we have

$$
\frac{2 u+1}{2 u} u^{2 k-1} s_{11}(u) \eta_{p}=\left(u-\gamma_{3}\right) \cdots\left(u-\gamma_{2 k+1}\right) \eta_{p}
$$

So, putting $u=\gamma_{1}-p+1$ in (5.6) we get

$$
p\left(\gamma_{1}+\gamma_{2}-p+1\right)\left(\gamma_{1}+\gamma_{3}-p+1\right) \cdots\left(\gamma_{1}+\gamma_{2 k+1}-p+1\right)=0
$$

However, this is impossible because of condition (5.3). Therefore, $p$ has to be equal to 0 and so, $\eta$ is a multiple of $\xi$.

To show that the submodule of $V$ generated by $\xi$ coincides with $V$ we consider the dual representation $V^{*}$ defined by

$$
(y \cdot f)(v)=f(\sigma(y) v), \quad y \in \mathrm{Y}^{+}(2), \quad f \in V^{*}, \quad v \in V
$$

where $\sigma$ is the antiautomorphism of $\mathrm{Y}^{+}(2)$ given by (1.26). One easily verifies that $V(\gamma)^{*} \simeq V(1-\gamma)$ and so, the representation $V^{*}$ is isomorphic to

$$
L^{\prime}\left(-\gamma_{1}, \gamma_{2}\right) \otimes \cdots \otimes L^{\prime}\left(-\gamma_{2 k-1}, \gamma_{2 k}\right) \otimes V\left(\gamma_{2 k+1}+1\right)
$$

where $L^{\prime}\left(-\gamma_{2 i-1}, \gamma_{2 i}\right)$ is the lowest weight representation of $\mathfrak{g l}(2)$; see (2.31).

Repeating the previous argument we prove that any nonzero submodule in $V^{*}$ contains the tensor product of the lowest weight vectors of the $L^{\prime}\left(-\gamma_{2 i-1}, \gamma_{2 i}\right)$ and the basis vector of $V\left(\gamma_{2 k+1}+1\right)$. The proof is completed exactly as that of Proposition 4.2.

The following proposition is proved in the same way as Proposition 4.3.

Proposition 5.3. Let $V(\mu(u))$ be an irreducible representation of $\mathrm{Y}^{+}(2)$ with the highest weight $\mu(u)$. Then $V(\mu(u))$ is finite-dimensional if and only if there exists a formal series $\psi(u) \in 1+u^{-2} \mathbb{C}\left[\left[u^{-2}\right]\right]$ such that $\left(1+1 / 2 u^{-1}\right) \psi(u) \mu(u)$ is a polynomial in $u^{-1}$ with a decomposition

$$
\left(1+\frac{1}{2} u^{-1}\right) \psi(u) \mu(u)=\left(1-\gamma_{1} u^{-1}\right) \cdots\left(1-\gamma_{2 k+1} u^{-1}\right)
$$

and $\gamma_{2 i-1}+\gamma_{2 i} \in \mathbb{Z}_{+}, i=1, \ldots, k$ for a certain re-enumeration of the $\gamma_{i}$.

By analogy with Theorem 4.4 we can reformulate these conditions in terms of monic polynomials in $u$. 
Theorem 5.4. The irreducible highest weight representation $V(\mu(u))$ of $\mathrm{Y}^{+}(2)$ is finite-dimensional if and only if there exist a monic polynomial $P(u) \in \mathbb{C}[u]$ with $P(u)=P(-u+1)$ and $\gamma \in \mathbb{C}$ such that $P(-\gamma) \neq 0$ and

$$
\frac{\mu(-u)}{\mu(u)}=\frac{P(u+1)}{P(u)} \frac{(u+\gamma)(2 u+1)}{(u-\gamma)(2 u-1)} .
$$

In this case the pair $(P(u), \gamma)$ is unique.

Proof. If $\operatorname{dim} V(\mu(u))<\infty$ then by Proposition 5.3 relation (5.8) holds for a certain even series $\psi(u)$. Re-enumerate the numbers $\gamma_{i}$ to satisfy condition (5.3). By Proposition 5.2 we have $\gamma_{2 i-1}+\gamma_{2 i} \in \mathbb{Z}_{+}$for $i=1, \ldots, k$ since $V(\mu(u))$ is finitedimensional. Define $P(u)$ as in the proof of Theorem 4.4 by $(4.30)-(4.32)$ and set $\gamma=\gamma_{k+1}$. The relation $P(-\gamma) \neq 0$ is implied by (5.3).

Conversely, let (5.9) be satisfied for a monic polynomial $P(u)$ such that $P(u)=$ $P(-u+1)$ and $\gamma \in \mathbb{C}$ with $P(-\gamma) \neq 0$. Let $\left\{-\delta_{1}, \ldots,-\delta_{s}, \delta_{1}+1, \ldots, \delta_{s}+1\right\}$ be the roots of $P(u)$; see the proof of Theorem 4.4. Set

$$
\begin{aligned}
\mu^{\prime}(u)= & \left(1-\left(\delta_{1}+1\right) u^{-1}\right) \cdots\left(1-\left(\delta_{s}+1\right) u^{-1}\right) \\
& \left(1+\delta_{1} u^{-1}\right) \cdots\left(1+\delta_{s} u^{-1}\right)\left(1-\gamma u^{-1}\right)\left(1+\frac{1}{2} u^{-1}\right)^{-1} .
\end{aligned}
$$

By Proposition 5.3 the representation $V\left(\mu^{\prime}(u)\right)$ is finite-dimensional. Since $\mu^{\prime}(u)$ satisfies $(5.9)$ the ratio $\mu(u) / \mu^{\prime}(u)$ is an even formal series in $u^{-1}$. This proves that $V(\mu(u))$ is also finite-dimensional.

To prove the uniqueness of the pair $(P(u), \gamma)$ suppose that $(R(u), \delta)$ is another pair, where $R(u)$ is a monic polynomial in $u$ such that $R(u)=R(-u+1), R(-\delta) \neq 0$ and

$$
\frac{P(u+1)}{P(u)} \frac{(u+\gamma)}{(u-\gamma)}=\frac{R(u+1)}{R(u)} \frac{(u+\delta)}{(u-\delta)}
$$

If $\gamma=\delta$ then we get $P(u)=R(u)$; see the proof of Theorem 2.8. Let $\gamma \neq \delta$. We prove by induction on the sum of the degrees of the polynomials $P(u)$ and $R(u)$ that (5.10) is impossible. If $P(u)=R(u)=1$ then this is obvious. Further, suppose $P(u)$ is of degree $\geq 2$. Take a root $u_{0}$ of $P(u)$ such that $u_{0}+1$ is not a root. Rewriting (5.10) in the form

$$
P(u) R(u+1)(u+\delta)(u-\gamma)=P(u+1) R(u)(u-\delta)(u+\gamma)
$$

we find that either $R\left(u_{0}\right)=0$, or $u_{0}=\delta$.

If $R\left(u_{0}\right)=0$ then we may write

$$
P(u)=P^{\prime}(u)\left(u-u_{0}\right)\left(u+u_{0}-1\right), \quad R(u)=R^{\prime}(u)\left(u-u_{0}\right)\left(u+u_{0}-1\right)
$$

and use the induction hypotheses for the polynomials $P^{\prime}(u)$ and $R^{\prime}(u)$. 
If $u_{0}=\delta$ but $R\left(u_{0}\right) \neq 0$ then we write

$$
P(u)=P^{\prime}(u)\left(u-u_{0}\right)\left(u+u_{0}-1\right)
$$

and obtain from (5.11) that

$$
P^{\prime}(u) R(u+1)\left(u+u_{0}-1\right)(u-\gamma)=P^{\prime}(u+1) R(u)\left(u-u_{0}+1\right)(u+\gamma) .
$$

Denote $\delta^{\prime}=u_{0}-1$. Then we have $\delta^{\prime} \neq \gamma$ because $P\left(-\delta^{\prime}\right)=0$. Moreover, $R\left(-\delta^{\prime}\right)=$ $R\left(u_{0}\right) \neq 0$ and we may apply the induction hypotheses to the polynomials $P^{\prime}(u)$ and $R(u)$.

Corollary 5.5. There is a one-to-one correspondence between finite-dimensional irreducible representations of the special twisted Yangian $\mathrm{SY}^{+}(2)$ and the pairs $(P(u), \gamma)$, where $P(u)$ is a monic polynomial in $u$ such that $P(u)=P(-u+1)$ and $\gamma \in \mathbb{C}$ with $P(-\gamma) \neq 0$. Every such representation is isomorphic to a representation of the form (5.4).

The following analogue of Corollary 4.7 holds.

Corollary 5.6. Consider an irreducible finite-dimensional representation of $\mathrm{Y}(2)$ of the form

$$
L\left(\gamma_{1},-\gamma_{2}\right) \otimes \cdots \otimes L\left(\gamma_{2 k-1},-\gamma_{2 k}\right)
$$

Then the representation

$$
L\left(\gamma_{1},-\gamma_{2}\right) \otimes \cdots \otimes L\left(\gamma_{2 k-1},-\gamma_{2 k}\right) \otimes V(-\gamma)
$$

of $\mathrm{Y}^{+}(2)$ is irreducible if and only if for each pair $1 \leq i<j \leq k$ the strings $S\left(\gamma_{2 i},-\gamma_{2 i-1}\right)$ and $S\left(\gamma_{2 j-1},-\gamma_{2 j}\right)$ are in general position, and for each $i=1, \ldots, k$ one has

$$
\gamma \notin S\left(\gamma_{2 i-1},-\gamma_{2 i}\right) \quad \text { and } \quad \gamma \notin S\left(\gamma_{2 i},-\gamma_{2 i-1}\right) \text {. }
$$

Proof. By Corollary 2.11, the strings $S\left(\gamma_{2 i-1},-\gamma_{2 i}\right), i=1, \ldots, k$ are pairwise in general position. Permuting the tensor factors in (5.12) if necessary, we may assume that $\gamma_{1}+\gamma_{2} \leq \cdots \leq \gamma_{2 k-1}+\gamma_{2 k}$. If the condition on the strings holds then one easily checks that (5.3) is satisfied for $\gamma_{2 k+1}=\gamma$ and so, by Proposition 5.2 representation (5.13) is irreducible.

To prove the 'only if' part consider first the case $k=1$. Let the representation $L\left(\gamma_{1},-\gamma_{2}\right) \otimes V(-\gamma)$ be irreducible but condition (5.14) is violated. Note that $L\left(\gamma_{2},-\gamma_{1}\right) \otimes V(-\gamma)$ is also irreducible. Indeed, these two representations have the same dimension and the tensor product of the highest weight vector of $L\left(\gamma_{2},-\gamma_{1}\right)$ and the basis vector of $V(-\gamma)$ generates a highest weight module with the same highest weight as $L\left(\gamma_{1},-\gamma_{2}\right) \otimes V(-\gamma)$. So we may assume that $\gamma \in S\left(\gamma_{1},-\gamma_{2}\right)$ 
and if $\gamma+\gamma_{1} \in \mathbb{Z}_{+}$then $\gamma+\gamma_{2} \leq \gamma+\gamma_{1}$. Then by Proposition 5.2 the representation $L\left(\gamma,-\gamma_{2}\right) \otimes V\left(-\gamma_{1}\right)$ is irreducible. It is isomorphic to $L\left(\gamma_{1},-\gamma_{2}\right) \otimes V(-\gamma)$ because they have the same highest weight. Comparing the dimensions we come to a contradiction.

Consider now the general case. Let representation (5.13) be irreducible. If (5.14) is violated then we move the corresponding tensor factor in (5.13) to the right to get the tensor product of the form $L\left(\gamma_{2 i-1},-\gamma_{2 i}\right) \otimes V(-\gamma)$. However, this representation is reducible. Contradiction.

Finally, replacing some of the tensor factors $L\left(\gamma_{2 i-1},-\gamma_{2 i}\right)$ in (5.13) respectively by $L\left(\gamma_{2 i},-\gamma_{2 i-1}\right)$ we get an isomorphic representation of $\mathrm{Y}^{+}(2)$ (see the proof of Corollary 4.7). Hence, the representation of $\mathrm{Y}(2)$ obtained from (5.12) by this replacement is also irreducible. Therefore, by Corollary 2.11 the corresponding strings are pairwise in general position.

Note that $V(\gamma)$ for $\gamma=1 / 2$ is the trivial representation of $\mathrm{Y}^{+}(2)$. Thus, in this particular case Corollary 5.6 provides a criterion of irreducibility of the restriction of (5.12) to $\mathrm{Y}^{+}(2)$.

Example 5.7. Consider the restriction of the representation $L\left(\gamma_{1},-\gamma_{2}\right)$ of $\mathrm{Y}(2)$ with $\gamma_{1}+\gamma_{2} \in \mathbb{Z}_{+}$to $\mathrm{Y}^{+}(2)$. By Corollary 5.6, it is reducible if and only if the string $S\left(\gamma_{1},-\gamma_{2}\right)$ contains $-1 / 2$. In this case $\gamma_{1}, \gamma_{2} \in 1 / 2+\mathbb{Z}_{+}$. Denote the highest weight vector of $L\left(\gamma_{1},-\gamma_{2}\right)$ by $\xi$ and set $\xi_{p}=\left(E_{1,-1}\right)^{p} \xi$. Suppose first that $\gamma_{1} \geq \gamma_{2}$. Then the following are $\mathrm{Y}^{+}(2)$-submodules in $L\left(\gamma_{1},-\gamma_{2}\right)$ :

$$
\begin{aligned}
& L_{1}=\text { span of }\left\{\xi_{0}, \ldots, \xi_{\gamma_{2}-1 / 2}\right\} \\
& L_{2}=\text { span of }\left\{\xi_{\gamma_{1}+1 / 2}, \ldots, \xi_{\gamma_{1}+\gamma_{2}}\right\}
\end{aligned}
$$

By Proposition 5.2 one has $L_{1} \simeq L\left(\gamma_{2},-1 / 2\right) \otimes V\left(-\gamma_{1}\right)$ and $L_{2} \simeq L\left(\gamma_{2},-1 / 2\right) \otimes$ $V\left(\gamma_{1}+1\right)$. The quotient $L\left(\gamma_{1},-\gamma_{2}\right) / L$, where $L=L_{1} \oplus L_{2}$, is isomorphic to $L\left(\gamma_{1}, \gamma_{2}+1\right)$.

If $\gamma_{1} \leq \gamma_{2}$ then $L\left(\gamma_{1},-\gamma_{2}\right)$ contains the submodule

$$
L=\text { span of }\left\{\xi_{\gamma_{1}+1 / 2}, \ldots, \xi_{\gamma_{2}-1 / 2}\right\}
$$

which is isomorphic to $L\left(\gamma_{2}, \gamma_{1}+1\right)$ and the quotient $L\left(\gamma_{1},-\gamma_{2}\right) / L$ is isomorphic to the direct sum of $L\left(\gamma_{1},-1 / 2\right) \otimes V\left(-\gamma_{2}\right)$ and $L\left(\gamma_{1},-1 / 2\right) \otimes V\left(\gamma_{2}+1\right)$.

Note that the mapping

$$
s_{i j}(u) \mapsto s_{i^{\prime} j^{\prime}}(u),
$$

where $i^{\prime}=-i$ for $i=-1,1$ and $i^{\prime}=i$ otherwise, defines an automorphism of the algebra $\mathrm{Y}^{+}(2 n)$. Consider the case $n=1$ and denote the composition of this automorphism with a finite-dimensional irreducible representation $V(\mu(u))$ of $\mathrm{Y}^{+}(2)$ by $V(\mu(u))^{\sharp}$. By Theorem 3.3, this is a representation with the highest weight which we denote by $\mu(u)^{\sharp}$. We shall say that $\mu(u)^{\sharp}$ is well-defined if $\mu(u)$ 
satisfies the conditions of Proposition 5.3. Since the automorphisms (1.29) and (5.15) commute, the ratio $\mu(u) / \mu(u)^{\sharp}$ takes the same value for all representations from the similarity class containing $V(\mu(u))$.

Equivalently, $V(\mu(u))$ contains a unique (up to scalar multiples) nonzero vector $\zeta$ such that $s_{1,-1}(u) \zeta=0$ (cf. Remark 3.4). Then $\zeta$ is an eigenvector for $s_{-1,-1}(u)$ and $\mu(u)^{\sharp}$ is defined by the formula

$$
s_{-1,-1}(u) \zeta=\mu(u)^{\sharp} \zeta .
$$

Proposition 5.8. Let $\mu(u)$ be a series given by (5.2) such that condition (5.3) is satisfied and $\gamma_{2 i-1}+\gamma_{2 i} \in \mathbb{Z}_{+}$for $i=1, \ldots, k$. Then

$$
\mu(u)^{\sharp}=\left(1-\gamma_{1} u^{-1}\right) \cdots\left(1-\gamma_{2 k} u^{-1}\right)\left(1+\left(\gamma_{2 k+1}+1\right) u^{-1}\right)\left(1+\frac{1}{2} u^{-1}\right)^{-1} .
$$

Proof. By Proposition 5.2 the representation $V(\mu(u))$ is isomorphic to the tensor product (5.4). In the notation of the proof of Proposition 5.2 set

$$
\zeta=\left(E_{1,-1}\right)^{\gamma_{1}+\gamma_{2}} \xi_{1} \otimes \cdots \otimes\left(E_{1,-1}\right)^{\gamma_{2 k-1}+\gamma_{2 k}} \xi_{k} \otimes \xi_{k+1} .
$$

Then using (1.31) we verify that $\zeta$ satisfies the relations

$$
s_{-1,-1}(u) \zeta=\left(1-\gamma_{1} u^{-1}\right) \cdots\left(1-\gamma_{2 k} u^{-1}\right)\left(1+\left(\gamma_{2 k+1}+1\right) u^{-1}\right)\left(1+\frac{1}{2} u^{-1}\right)^{-1} \zeta
$$

and $s_{1,-1}(u) \zeta=0$. Thus, $V(\mu(u))^{\sharp}$ is isomorphic to $V\left(\mu(u)^{\sharp}\right)$ with $\mu(u)^{\sharp}$ given by (5.17).

The following theorem together with Theorem 3.3 gives a description of finitedimensional irreducible representations of $\mathrm{Y}^{+}(2 n)$ (cf. Theorem 4.8). We use notation (2.35) and (4.34).

Theorem 5.9. The irreducible highest weight representation $V(\mu(u)), \mu(u)=$ $\left(\mu_{1}(u), \ldots, \mu_{n}(u)\right)$ of $\mathrm{Y}^{+}(2 n), n \geq 2$ is finite-dimensional if and only if $\mu_{1}(u)^{\#}$ is well-defined and either one of the following four relations holds:

$$
\begin{aligned}
\mu_{1}(-u) & \Rightarrow \mu_{1}(u) \rightarrow \mu_{2}(u) \rightarrow \cdots \rightarrow \mu_{n}(u), \\
\frac{2 u-1}{2 u+1} \mu_{1}(-u) & \Rightarrow \mu_{1}(u) \rightarrow \mu_{2}(u) \rightarrow \cdots \rightarrow \mu_{n}(u), \\
\mu_{1}(-u)^{\sharp} & \Rightarrow \mu_{1}(u)^{\sharp} \rightarrow \mu_{2}(u) \rightarrow \cdots \rightarrow \mu_{n}(u), \\
\frac{2 u-1}{2 u+1} \mu_{1}(-u)^{\sharp} & \Rightarrow \mu_{1}(u)^{\sharp} \rightarrow \mu_{2}(u) \rightarrow \cdots \rightarrow \mu_{n}(u) .
\end{aligned}
$$


Proof. Suppose that $\operatorname{dim} V(\mu(u))<\infty$. Then by Proposition 3.5 we have

$$
\mu_{1}(u) \rightarrow \mu_{2}(u) \rightarrow \cdots \rightarrow \mu_{n}(u)
$$

Denote the composition of $V(\mu(u))$ with the automorphism $(5.15)$ by $V(\mu(u))^{\sharp}$. By Theorem 3.3 the representation $V(\mu(u))^{\#}$ is highest weight. To find its highest weight vector consider the cyclic span $V=\mathrm{Y}^{+}(2) \xi$, where $\xi$ is the highest weight vector of $V(\mu(u))$, and $\mathrm{Y}^{+}(2)$ is identified with the subalgebra in $\mathrm{Y}^{+}(2 n)$ generated by the coefficients of the series $s_{i j}(u)$ with $i, j=-1,1$. We claim that $V$ is an irreducible $\mathrm{Y}^{+}(2)$-module. Indeed, let $J$ be the left ideal in $\mathrm{Y}^{+}(2 n)$ generated by the $s_{k l}^{(r)}$ with $k<l$ and $(k, l) \neq(-1,1)$. Then $V$ is annihilated by $J$. This follows from the fact that $J \xi=0$ and the relations

$$
\left[s_{i j}(u), s_{k l}(v)\right] \equiv 0 \quad \bmod J
$$

where $i, j= \pm 1$ and $k<l,(k, l) \neq(-1,1)$. To verify (5.23) we may assume, applying (1.18) if necessary, that $l \geq 2$. However, in this case (5.23) is immediate from (1.17). Now, if $\widetilde{V}$ is a nonzero submodule in $V$ then there exists a nonzero vector $\eta \in \widetilde{V}$ such that $s_{-1,1}(u) \eta=0$. Then $s_{k l}(u) \eta=0$ for all $k<l$ since $V$ is annihilated by $J$. So, $\eta$ is a multiple of $\xi$; see Remark 3.4. This proves that $\widetilde{V}=V$.

Thus, $V$ is isomorphic to the representation $V\left(\mu_{1}(u)\right)$ of $\mathrm{Y}^{+}(2)$. Since $\operatorname{dim} V<$ $\infty$ there exists a nonzero vector $\zeta \in V$ such that

$$
s_{-1,-1}(u) \zeta=\mu_{1}(u)^{\sharp} \zeta \quad \text { and } \quad s_{1,-1}(u) \zeta=0 ;
$$

see (5.16). Moreover, we have $s_{k k}(u) \zeta=\mu_{k}(u) \zeta$ for $k=2, \ldots, n$. This is implied by the following relations which are immediate from (1.17):

$$
\left[s_{i j}(u), s_{k k}(v)\right] \equiv 0 \bmod J
$$

where $i, j= \pm 1$ and $k \geq 2$. Since $\zeta$ is annihilated by $J$ we may conclude that $\zeta$ is the highest weight vector of $V(\mu(u))^{\sharp}$ and so, the latter is a representation with the highest weight $\left(\mu_{1}(u)^{\sharp}, \mu_{2}(u), \ldots, \mu_{n}(u)\right)$. Applying Proposition 3.5 again, we find that

$$
\mu_{1}(u)^{\sharp} \rightarrow \mu_{2}(u) \rightarrow \cdots \rightarrow \mu_{n}(u) .
$$

Further, since the representation $V\left(\mu_{1}(u)\right)$ of $\mathrm{Y}^{+}(2)$ is finite-dimensional, then by Proposition 5.3 we may assume, applying an automorphism of the form (1.29) if necessary, that $\left(1+1 / 2 u^{-1}\right) \mu_{1}(u)$ is a polynomial in $u^{-1}$. By (5.22) we have

$$
\frac{\mu_{1}(u)}{\mu_{2}(u)}=\frac{P(u+1)}{P(u)}
$$


for a monic polynomial $P(u)$ in $u$. Hence, applying an automorphism (1.29) again, we may assume that both $\left(1+1 / 2 u^{-1}\right) \mu_{1}(u)$ and $\left(1+1 / 2 u^{-1}\right) \mu_{2}(u)$ are polynomials in $u^{-1}$ :

$$
\begin{aligned}
& \mu_{1}(u)=\left(1-\gamma_{1} u^{-1}\right) \cdots\left(1-\gamma_{2 k+1} u^{-1}\right)\left(1+\frac{1}{2} u^{-1}\right)^{-1} \\
& \mu_{2}(u)=\left(1-\delta_{1} u^{-1}\right) \cdots\left(1-\delta_{2 k+1} u^{-1}\right)\left(1+\frac{1}{2} u^{-1}\right)^{-1}
\end{aligned}
$$

Re-enumerating the $\gamma_{i}$ if necessary, we may assume that condition (5.3) holds. Moreover, $\gamma_{2 i-1}+\gamma_{2 i} \in \mathbb{Z}_{+}$for $i=1, \ldots, k$ since $\operatorname{dim} V\left(\mu_{1}(u)\right)<\infty$; see Proposition 5.2. Further, (5.25) implies that there exists a re-enumeration of the $\delta_{i}$ such that

$$
\delta_{i}-\gamma_{i} \in \mathbb{Z}_{+} \quad \text { for } \quad i=1, \ldots, 2 k+1 .
$$

To see this, choose a string-type subset $\{a+1, a+2, \ldots, b\}$ of roots of $P(u)$ such that $a$ and $b+1$ are not roots. Then we find from (5.25) that $\gamma_{i}=a$ for a certain $i$, and $b$ equals one of the $\delta$ 's which we re-denote by $\delta_{i}$. Therefore, $\delta_{i}-\gamma_{i} \in \mathbb{Z}_{+}$and we can write

$$
\mu_{1}(u)=\left(1-a u^{-1}\right) \mu_{1}^{\prime}(u), \quad \mu_{2}(u)=\left(1-b u^{-1}\right) \mu_{2}^{\prime}(u)
$$

and

$$
\frac{P(u+1)}{P(u)}=\frac{P^{\prime}(u+1)}{P^{\prime}(u)} \cdot \frac{u-a}{u-b},
$$

where $P(u)=(u-a-1) \cdots(u-b) P^{\prime}(u)$. Then we proceed by induction applying the same argument to the series $\mu_{1}^{\prime}(u), \mu_{2}^{\prime}(u)$ and the polynomial $P^{\prime}(u)$.

Now we use Proposition 5.8. Exactly as above, we obtain from (5.24) that there exists a permutation $\pi \in \mathfrak{S}_{2 k+1}$ such that

$$
\delta_{\pi(i)}-\gamma_{i} \in \mathbb{Z}_{+}, \quad i=1, \ldots, 2 k \quad \text { and } \quad \delta_{\pi(2 k+1)}+\gamma_{2 k+1}+1 \in \mathbb{Z}_{+} .
$$

Now, (5.26) and (5.27) imply that $\delta_{\pi(i)}-\delta_{i} \in \mathbb{Z}$ for $i=1, \ldots, 2 k$. But then this has to be true for $i=2 k+1$ as well. Thus, $2 \gamma_{2 k+1}+1 \in \mathbb{Z}$, that is, the number $\gamma:=\gamma_{2 k+1}$ belongs to $\frac{1}{2} \mathbb{Z}$.

Suppose first that $\gamma \in \frac{1}{2}+\mathbb{Z}$ and $\gamma \geq-\frac{1}{2}$. Then exactly as in the proof of Theorem 4.4 we obtain that $\mu_{1}(-u) \Rightarrow \mu_{1}(u)$. Similarly, if $\gamma \in \frac{1}{2}+\mathbb{Z}$ and $\gamma \leq-\frac{1}{2}$ then $\mu_{1}(-u)^{\sharp} \Rightarrow \mu_{1}(u)^{\sharp}$. Further, let $\gamma \in \mathbb{Z}$ and $\gamma \geq 0$. Then writing

$$
\left(1+\frac{1}{2} u^{-1}\right) \mu_{1}(u)=\left(1-\gamma_{1} u^{-1}\right) \cdots\left(1-\gamma_{2 k} u^{-1}\right)\left(1-\gamma u^{-1}\right)\left(1-0 u^{-1}\right)
$$

we see that $\left(1-1 / 2 u^{-1}\right) \mu_{1}(-u) \Rightarrow\left(1+1 / 2 u^{-1}\right) \mu_{1}(u)$, or, equivalently,

$$
\frac{2 u-1}{2 u+1} \mu_{1}(-u) \Rightarrow \mu_{1}(u) \text {. }
$$


Similarly, if $\gamma \in \mathbb{Z}$ and $\gamma \leq-1$ then using Proposition 5.8 we obtain

$$
\frac{2 u-1}{2 u+1} \mu_{1}(-u)^{\sharp} \Rightarrow \mu_{1}(u)^{\sharp} \text {. }
$$

Combining these relations with (5.22) or (5.24) we complete the proof of the 'only if' part.

Suppose now that relation (5.18) is satisfied. Then we repeat the corresponding argument of the proof of Theorem 4.8 to show that $V(\mu(u))$ is finite-dimensional. Similarly, if (5.20) holds then the irreducible representation with the highest weight $\left(\mu_{1}(u)^{\sharp}, \mu_{2}(u), \ldots, \mu_{n}(u)\right)$ is finite-dimensional, and so $V(\mu(u))$ is, since the automorphism (5.15) is involutive.

If (5.19) holds then

$$
\frac{\mu_{1}(-u)}{\mu_{1}(u)}=\frac{P_{1}(u+1)}{P_{1}(u)} \cdot \frac{2 u+1}{2 u-1}
$$

for a monic polynomial $P_{1}(u)$ of the form (4.41), and relations (4.39) hold for polynomials $P_{i}(u)$ of the form (4.40). Define the $\lambda_{j}(u)$ by (4.42) and (4.43). Then the representation $L(\lambda(u))$ of $\mathrm{Y}(2 n)$ is finite-dimensional by Theorem 2.12.

Let $V\left(\mu_{0}\right)$ denote the irreducible representation of the Lie algebra $\mathfrak{o}(2 n)$ with the highest weight $\mu_{0}=(-1 / 2, \ldots,-1 / 2)$. It is finite-dimensional; see $(3.6)$ and (3.7). Extend $V\left(\mu_{0}\right)$ to a representation of $\mathrm{Y}^{+}(2 n)$ by using (1.23) and consider the tensor product $L(\lambda(u)) \otimes V\left(\mu_{0}\right)$. The tensor product of the highest weight vectors of $L(\lambda(u))$ and $V\left(\mu_{0}\right)$ generates a $\mathrm{Y}^{+}(2 n)$-submodule with the highest weight $\mu^{\prime}(u)=\left(\mu_{1}^{\prime}(u), \ldots, \mu_{n}^{\prime}(u)\right)$ where $\mu_{i}^{\prime}(u)=\lambda_{i}(u) \lambda_{-i}(-u)\left(1+1 / 2 u^{-1}\right)^{-1}$; see $(3.10)$ and (3.11). So the representation $V\left(\mu^{\prime}(u)\right)$ of $\mathrm{Y}^{+}(2 n)$ is finite-dimensional. However, the polynomials $\mu_{i}^{\prime}(u)$ satisfy (5.28) and (4.39). Hence, there exists an automorphism of $\mathrm{Y}^{+}(2 n)$ of the form (1.29) such that its composition with the representation $V\left(\mu^{\prime}(u)\right)$ is isomorphic to $V(\mu(u))$ which completes the proof in this case.

Finally, if relation (5.21) holds then the same argument shows that the representation $V\left(\mu_{1}(u)^{\sharp}, \mu_{2}(u), \ldots, \mu_{n}(u)\right)$ is finite-dimensional, and so $V(\mu(u))$ is.

We conclude this section with the following description of representations of the special twisted Yangian $\mathrm{SY}^{+}(2 n)$ implied by Theorem 5.9 (cf. Corollary 4.9).

Corollary 5.10. There is a one-to-one correspondence between finite-dimensional irreducible representations of the special twisted Yangian $\mathrm{SY}^{+}(2 n), n \geq 2$ and the families $\left\{P_{1}(u), \ldots, P_{n}(u), \varepsilon\right\}$, where the $P_{i}(u)$ are monic polynomials in $u$ with $P_{1}(u)=P_{1}(-u+1)$, and the parameter $\varepsilon$ takes values in $\{1,2,3,4\}$; the values $\varepsilon=1$ and $\varepsilon=3$ are identified if $P_{1}(1 / 2) \neq 0$. Every such representation is isomorphic to a subquotient of a representation of the form $L(\lambda(u))$ or $L(\lambda(u)) \otimes V\left(\mu_{0}\right)$.

Proof. Each of the four conditions (5.18)-(5.21) allows one to naturally associate a family $\left\{P_{1}(u), \ldots, P_{n}(u)\right\}$ of monic polynomials to the corresponding similarity 
class of finite-dimensional irreducible representations of $\mathrm{Y}^{+}(2 n)$; see the proof of Theorem 5.9. We associate the values of $\varepsilon$ respectively to conditions (5.18)-(5.21). It follows from the proof of Theorem 5.9 that two families of monic polynomials with values of $\varepsilon=1$ and $\varepsilon=3$ correspond to the same similarity class if and only if $P_{1}(1 / 2) \neq 0$. One easily checks that identifying these values we obtain the desired one-to-one correspondence. The second claim also follows from the proof of Theorem 5.9 .

\section{Representations of $\mathrm{Y}^{+}(2 n+1)$}

The description of finite-dimensional irreducible representations of $\mathrm{Y}^{+}(2 n+1)$ is based on the simplest nontrivial case $n=1$. Our main instrument in this case is an investigation of the restriction of an irreducible highest weight representation of $\mathrm{Y}^{+}(3)$ to the subalgebra $\mathrm{Y}^{+}(2)$ and the use of the results of the previous section.

Irreducible highest weight representations $V(\mu(u))$ of $\mathrm{Y}^{+}(3)$ are parameterized by pairs of formal series $\mu(u)=\left(\mu_{0}(u), \mu_{1}(u)\right)$, where $\mu_{0}(u) \in 1+u^{-2} \mathbb{C}\left[\left[u^{-2}\right]\right]$ and $\mu_{1}(u) \in 1+u^{-1} \mathbb{C}\left[\left[u^{-1}\right]\right]$.

Proposition 6.1. If $\operatorname{dim} V(\mu(u))<\infty$ then there exists a formal series $\varphi(u) \in$ $1+u^{-1} \mathbb{C}\left[\left[u^{-1}\right]\right]$ such that $\varphi(u) \mu_{0}(u)$ and $\varphi(u) \mu_{1}(u)$ are polynomials in $u^{-1}$.

Proof. Denote by $\xi$ the highest weight vector of $V(\mu(u))$. Let $k$ denote the minimum nonnegative integer such that $s_{10}^{(k+1)} \xi$ is a linear combination of the vectors $\xi_{1}:=$ $s_{10}^{(1)} \xi, \ldots, \xi_{k}:=s_{10}^{(k)} \xi$. Relations (1.17) give

$$
\begin{aligned}
& {\left[s_{11}(u), s_{10}(v)\right]=\frac{1}{u-v}\left(s_{10}(v) s_{11}(u)-s_{10}(u) s_{11}(v)\right)} \\
& \quad+\frac{u-v+1}{u^{2}-v^{2}} s_{1,-1}(v) s_{01}(u)-\frac{1}{u+v} s_{1,-1}(u) s_{-1,0}(v)-\frac{1}{u^{2}-v^{2}} s_{1,-1}(u) s_{01}(v) .
\end{aligned}
$$

Since the vector $\xi$ is annihilated by $s_{01}(u)$ (and hence by $s_{-1,0}(u)$ ) this makes the consideration here very similar to the case of $\mathrm{Y}(2)$; see Proposition 2.5. In particular, repeating the corresponding argument of the proof of Proposition 2.5 we can show that

$$
s_{10}(u) \xi=a_{1}(u) \xi_{1}+\cdots+a_{k}(u) \xi_{k}
$$

for certain series $a_{i}(u)$; cf. (2.13). By (1.17) we have

$$
\begin{aligned}
{\left[s_{00}(u), s_{10}(v)\right] } & =\frac{u+v+1}{u^{2}-v^{2}} s_{10}(u) s_{00}(v) \\
& -\frac{1}{u+v} s_{0,-1}(u) s_{00}(v)-\frac{2 v+1}{u^{2}-v^{2}} s_{10}(v) s_{00}(u) .
\end{aligned}
$$


Taking the coefficients at $u^{-2}$ in (6.1) and (6.2) and using again the argument of the proof of Proposition 2.5 we find that the components of $\mu(u)$ can be written in the form

$$
\mu_{0}(u)=B(u) a_{k}(u) \quad \text { and } \quad \mu_{1}(u)=C(u) a_{k}(u),
$$

where $B(u)$ and $C(u)$ are monic polynomials in $u$ of degree $k$. Thus, the series $\varphi(u)$ can be defined by $\varphi(u)=\left(a_{k}(u) u^{k}\right)^{-1}$.

Write the polynomials $\varphi(u) \mu_{0}(u)$ and $\varphi(u) \mu_{1}(u)$ in the form

$$
\begin{aligned}
& \varphi(u) \mu_{0}(u)=\left(1+\alpha_{1} u^{-1}\right) \cdots\left(1+\alpha_{k} u^{-1}\right), \\
& \varphi(u) \mu_{1}(u)=\left(1+\beta_{1} u^{-1}\right) \cdots\left(1+\beta_{k} u^{-1}\right),
\end{aligned}
$$

with $\alpha_{i}, \beta_{i} \in \mathbb{C}$. Since $\mu_{0}(u)$ is an even series in $u^{-1}$ we can find an appropriate automorphism of the form (1.29) such that its composition with $V(\mu(u))$ is an irreducible representation with the following highest weight which we shall again denote by $\mu(u)$ :

$$
\begin{aligned}
& \mu_{0}(u)=\left(1-\alpha_{1}^{2} u^{-2}\right) \cdots\left(1-\alpha_{k}^{2} u^{-2}\right), \\
& \mu_{1}(u)=\left(1-\alpha_{1} u^{-1}\right) \cdots\left(1-\alpha_{k} u^{-1}\right)\left(1+\beta_{1} u^{-1}\right) \cdots\left(1+\beta_{k} u^{-1}\right) .
\end{aligned}
$$

Our next task is to find necessary conditions for the $\alpha_{i}$ and $\beta_{i}$ so that the representation $V(\mu(u))$ with the highest weight given by $(6.4)$ be finite-dimensional.

Consider the following representation of $\mathrm{Y}(3)$ :

$$
L\left(\alpha_{1}, \alpha_{1}, \beta_{1}\right) \otimes \cdots \otimes L\left(\alpha_{k}, \alpha_{k}, \beta_{k}\right) .
$$

It follows from (1.15) that the $\mathrm{Y}^{+}(3)$-cyclic span of the tensor product of the highest weight vectors of the $L\left(\alpha_{i}, \alpha_{i}, \beta_{i}\right)$ is a representation of $\mathrm{Y}^{+}(3)$ with the highest weight $\mu(u)=\left(\mu_{0}(u), \mu_{1}(u)\right)$ given by (6.4). The generators $t_{i j}^{(r)}$ of $\mathrm{Y}(3)$ with $r \geq k+1$ act trivially in representation (6.5) (cf. (2.18)). Therefore, by (1.15) the generators $s_{i j}^{(r)}$ of $\mathrm{Y}^{+}(3)$ with $r \geq 2 k+1$ act trivially in $V(\mu(u))$. The series

$$
S_{i j}(u):=u^{2 k} s_{i j}(u)
$$

is then a polynomial operator in $u$ and we may evaluate $u$ to get well-defined operators in $V(\mu(u))$. Set

$$
\begin{aligned}
& a_{0}(u)=u^{2 k} \mu_{0}(u)=\left(u^{2}-\alpha_{1}^{2}\right) \cdots\left(u^{2}-\alpha_{k}^{2}\right), \\
& a_{1}(u)=u^{2 k} \mu_{1}(u)=\left(u-\alpha_{1}\right) \cdots\left(u-\alpha_{k}\right)\left(u+\beta_{1}\right) \cdots\left(u+\beta_{k}\right) .
\end{aligned}
$$

Using Proposition 1.1 we shall now equip the vector space $V:=V(\mu(u))$ with another structure of $\mathrm{Y}^{+}(3)$-module. The matrix elements of the Sklyanin comatrix $\widehat{S}(u)$ can be found from [M3, Section 6]. In particular,

$$
\begin{aligned}
\widehat{s}_{00}(u) & =s_{11}(-u) s_{11}(u-1)-s_{1,-1}(-u) s_{-1,1}(u-1), \\
\widehat{s}_{-1,0}(u) & =s_{0,-1}(-u) s_{-1,1}(u-1)-s_{01}(-u) s_{11}(u-1), \\
\widehat{s}_{11}(u) & =s_{11}(-u) s_{00}(u-1)-s_{10}(-u) s_{-1,0}(u-1) .
\end{aligned}
$$


Let us take the composition of the representation $V(\mu(u))$ with the automorphism $S(u) \rightarrow \widehat{S}(-u+1 / 2)$ of $\mathrm{Y}^{+}(3)$; see (1.27). One can easily see from (6.8) that the resulting representation is highest weight and generated by the vector $\xi$. The highest weight of this representation is

$$
\left(\mu_{1}(u-1 / 2) \mu_{1}(-u-1 / 2), \mu_{1}(u-1 / 2) \mu_{0}(-u-1 / 2)\right) .
$$

We shall use the notation

$$
\alpha_{i}^{*}=\frac{1}{2}-\beta_{i}, \quad \beta_{i}^{*}=\frac{1}{2}-\alpha_{i}, \quad \text { for } \quad i=1, \ldots, k .
$$

Set

$$
\begin{aligned}
& \mu_{0}^{*}(u)=\left(1-\left(\alpha_{1}^{*}\right)^{2} u^{-2}\right) \cdots\left(1-\left(\alpha_{k}^{*}\right)^{2} u^{-2}\right) \\
& \mu_{1}^{*}(u)=\left(1-\alpha_{1}^{*} u^{-1}\right) \cdots\left(1-\alpha_{k}^{*} u^{-1}\right)\left(1+\beta_{1}^{*} u^{-1}\right) \cdots\left(1+\beta_{k}^{*} u^{-1}\right) .
\end{aligned}
$$

Take the composition of the irreducible representation of $\mathrm{Y}^{+}(3)$ with the highest weight (6.9) and the automorphism (1.29) corresponding to the series

$$
\psi_{0}(u)=\frac{\mu_{0}^{*}(u)}{\mu_{1}(u-1 / 2) \mu_{1}(-u-1 / 2)} .
$$

The resulting representation is isomorphic to $V\left(\mu^{*}(u)\right), \mu^{*}(u)=\left(\mu_{0}^{*}(u), \mu_{1}^{*}(u)\right)$.

Thus, the vector space $V$ carries two representations of $\mathrm{Y}^{+}(3)$ isomorphic to $V(\mu(u))$ and $V\left(\mu^{*}(u)\right)$, respectively. To distinguish them we shall denote by $s_{i j}^{*}(u)$ the action of the generators $s_{i j}(u)$ in $V\left(\mu^{*}(u)\right)$. By the above definition we have

$$
s_{i j}^{*}(u)=\psi_{0}(u) \widehat{s}_{i j}\left(-u+\frac{1}{2}\right)
$$

Denote by $S_{i j}^{*}(u)$ the corresponding polynomial operator $(6.6)$ in $V\left(\mu^{*}(u)\right)$. By analogy with (6.7) set

$$
a_{0}^{*}(u)=u^{2 k} \mu_{0}^{*}(u), \quad a_{1}^{*}(u)=u^{2 k} \mu_{1}^{*}(u) .
$$

For the proof of the next proposition fix an index $i \in\{1, \ldots, k\}$ and set $\alpha:=\alpha_{i}$, $\beta^{*}:=\frac{1}{2}-\alpha_{i}$. Given nonnegative integer $p$ introduce the following vector in $V$ :

$$
\eta_{p}=S_{10}(-\alpha+p-1) \cdots S_{10}(-\alpha) \xi .
$$


Proposition 6.2. We have the relations

$$
\begin{aligned}
S_{00}(u) \eta_{p} & =a_{0}(u) \frac{u^{2}-(\alpha-p)^{2}}{u^{2}-\alpha^{2}} \eta_{p}, \\
S_{-1,0}(u) \eta_{p} & =p a_{0}(u) a_{1}(-\alpha+p-1) \frac{u+\alpha-p}{u^{2}-\alpha^{2}} \eta_{p-1}, \\
S_{-1,1}^{*}(u) \eta_{p} & =0 \\
S_{11}^{*}(u) \eta_{p} & =a_{1}^{*}(u) \frac{u+\beta^{*}+p}{u+\beta^{*}} \eta_{p} .
\end{aligned}
$$

Proof. We use induction on $p$ to prove (6.13)-(6.16) simultaneously. For $p=0$ all the relations are obvious. Suppose now that $p \geq 1$. Using the induction hypotheses and (6.2) we obtain

$$
\begin{aligned}
S_{00}(u) \eta_{p} & =S_{00}(u) S_{10}(-\alpha+p-1) \eta_{p-1} \\
& =\frac{u^{2}-(\alpha-p)^{2}}{u^{2}-(\alpha-p+1)^{2}} S_{10}(-\alpha+p-1) S_{00}(u) \eta_{p-1}
\end{aligned}
$$

which proves (6.13). Further, (1.28) gives

$$
\left[\widehat{s}_{11}(u), s_{10}(v)\right]=-\frac{1}{u-v-2}\left(\widehat{s}_{1,-1}(u) s_{-1,0}(v)+\widehat{s}_{10}(u) s_{00}(v)+\widehat{s}_{11}(u) s_{10}(v)\right) .
$$

Hence, using the induction hypotheses and (6.12) we can write

$$
\begin{aligned}
S_{11}^{*}(u) \eta_{p} & =S_{11}^{*}(u) S_{10}(-\alpha+p-1) \eta_{p-1} \\
& =\frac{u+\beta^{*}+p}{u+\beta^{*}+p-1} S_{10}(-\alpha+p-1) S_{11}^{*}(u) \eta_{p-1},
\end{aligned}
$$

which proves (6.16). The proof of $(6.15)$ is the same; it suffices to use the expansion for $\left[\widehat{s}_{-1,1}(u), s_{10}(v)\right]$ implied by (1.28). Finally, to prove $(6.14)$ consider the following relation implied by (1.17):

$$
\begin{aligned}
& {\left[s_{-1,0}(u), s_{10}(v)\right]=\frac{1}{u-v}\left(s_{10}(u) s_{-1,0}(v)-s_{10}(v) s_{-1,0}(u)\right)} \\
& \quad+\frac{u-v-1}{u^{2}-v^{2}} s_{11}(v) s_{00}(u)-\frac{1}{u+v} s_{-1,-1}(u) s_{00}(v)+\frac{1}{u^{2}-v^{2}} s_{11}(u) s_{00}(v) .
\end{aligned}
$$

Using the induction hypotheses we obtain

$$
\begin{aligned}
S_{-1,0}(u) \eta_{p} & =S_{-1,0}(u) S_{10}(-\alpha+p-1) \eta_{p-1} \\
& =\frac{u+\alpha-p}{u+\alpha-p+1} S_{10}(-\alpha+p-1) S_{-1,0}(u) \eta_{p-1} \\
& +\frac{u+\alpha-p}{u^{2}-(\alpha-p+1)^{2}} S_{11}(-\alpha+p-1) S_{00}(u) \eta_{p-1}
\end{aligned}
$$


Using (6.13) and applying further (6.19) to $S_{-1,0}(u) \eta_{p-1}$ etc. we find that

$$
S_{-1,0}(u) \eta_{p}=a_{0}(u) \frac{u+\alpha-p}{u^{2}-\alpha^{2}} \eta_{p}^{(1)}
$$

where

$$
\eta_{p}^{(1)}=\sum_{q=1}^{p} S_{10}(-\alpha+p-1) \cdots S_{11}(-\alpha+q-1) \cdots S_{10}(-\alpha) \xi
$$

and $S_{11}(-\alpha+q-1)$ takes the $q$ th position from the right.

On the other hand, by (6.8)

$$
\widehat{s}_{11}(u+1)=s_{11}(-u-1) s_{00}(u)-s_{10}(-u-1) s_{-1,0}(u) .
$$

Let us multiply both sides of this relation by $(u(u+1))^{2 k}$ and apply them to the vector $\eta_{p-1}$. By $(6.12)$ we have

$$
\widehat{s}_{11}(u+1)=s_{11}^{*}\left(-u-\frac{1}{2}\right) \psi_{0}\left(u+\frac{1}{2}\right)^{-1} .
$$

So, we can use $(6.16)$ to calculate $(u(u+1))^{2 k} \widehat{s}_{11}(u+1) \eta_{p-1}$. Performing this calculation and using (6.13) and (6.20) we come to the relation

$$
a_{1}(-u-1) \eta_{p-1}=\frac{u-\alpha+p-1}{u-\alpha} S_{11}(-u-1) \eta_{p-1}-\frac{1}{u-\alpha} S_{10}(-u-1) \eta_{p-1}^{(1)}
$$

Note that

$$
\eta_{p}^{(1)}=S_{11}(-\alpha+p-1) \eta_{p-1}+S_{10}(-\alpha+p-1) \eta_{p-1}^{(1)} .
$$

Therefore, putting $u=\alpha-p$ into (6.21) we get

$$
\eta_{p}^{(1)}=p a_{1}(-\alpha+p-1) \eta_{p-1}
$$

Together with (6.20) this completes the proof of (6.14).

Given nonnegative integers $p_{1}, \ldots, p_{k}$ introduce vectors in $V$ of a more general form:

$$
\eta_{p_{1}, \ldots, p_{k}}=\left(\prod_{i=1, \ldots, k}^{\rightarrow} S_{10}\left(-\alpha_{i}+p_{i}-1\right) \cdots S_{10}\left(-\alpha_{i}\right)\right) \xi
$$

Set

$$
S_{-1,0}^{\natural}(u)=\frac{S_{-1,0}(u)}{\left(u+\alpha_{1}-p_{1}\right) \cdots\left(u+\alpha_{k}-p_{k}\right)} .
$$


Proposition 6.3. (i) The series $S_{-1,0}^{\natural}(u) \eta_{p_{1}, \ldots, p_{k}}$ is a polynomial in $u$ with values in $V$ and for every $i=1, \ldots, k$

$$
S_{-1,0}^{\natural}\left(\alpha_{i}-p_{i}\right) \eta_{p_{1}, \ldots, p_{k}}=-a_{1}\left(-\alpha_{i}+p_{i}-1\right) \prod_{j=1}^{k}\left(\alpha_{i}-\alpha_{j}-p_{i}\right) \eta_{p_{1}, \ldots, p_{i}-1, \ldots, p_{k}} .
$$

(ii) One has the relation

$$
S_{00}(u) \eta_{p_{1}, \ldots, p_{k}}=\left(u^{2}-\left(\alpha_{1}-p_{1}\right)^{2}\right) \cdots\left(u^{2}-\left(\alpha_{k}-p_{k}\right)^{2}\right) \eta_{p_{1}, \ldots, p_{k}} .
$$

Proof. First we verify (ii) by using (6.2) and repeating the corresponding argument of the proof of Proposition 6.2. Next, by an obvious induction with the use of (6.18) and (6.25) (cf. the proof of (6.20)) the show that

$$
\begin{aligned}
& S_{-1,0}(u) \eta_{p_{1}, \ldots, p_{k}}=\left(u+\alpha_{1}-p_{1}\right) \cdots\left(u+\alpha_{k}-p_{k}\right) \\
\times & \sum_{j=1}^{k}\left(u-\alpha_{1}\right) \cdots\left(u-\alpha_{j-1}\right)\left(u-\alpha_{j+1}+p_{j+1}\right) \cdots\left(u-\alpha_{k}+p_{k}\right) \eta_{p_{1}, \ldots, p_{k}}^{(j)}
\end{aligned}
$$

where $\eta_{p_{1}, \ldots, p_{k}}^{(j)}$ is the vector in $V$ obtained from $\eta_{p_{1}, \ldots, p_{k}}$ by replacing the product

$$
S_{10}\left(-\alpha_{j}+p_{j}-1\right) \cdots S_{10}\left(-\alpha_{j}\right)
$$

in $(6.23)$ with the sum

$$
\sum_{q=1}^{p_{j}} S_{10}\left(-\alpha_{j}+p_{j}-1\right) \cdots S_{11}\left(-\alpha_{j}+q-1\right) \cdots S_{10}\left(-\alpha_{j}\right)
$$

here $S_{11}\left(-\alpha_{j}+q-1\right)$ takes the $q$ th position from the right. This proves that the series $S_{-1,0}^{\natural}(u) \eta_{p_{1}, \ldots, p_{k}}$ is a polynomial in $u$. Moreover, (6.26) and (6.22) imply (6.24) for $i=k$. To complete the proof we show that $\eta_{p_{1}, \ldots, p_{k}}$ is not changed if we arbitrary permute the products (6.27) in the definition (6.23). We only need to show this for permutations of adjacent products. By (6.25) it suffices to verify the following auxiliary claim: if a vector $\eta \in V$ satisfies

$$
S_{00}(u) \eta=\left(u^{2}-\gamma_{1}^{2}\right) \cdots\left(u^{2}-\gamma_{k}^{2}\right) \eta
$$

then

$$
S_{10}\left(\gamma_{1}\right) S_{10}\left(\gamma_{2}\right) \eta=S_{10}\left(\gamma_{2}\right) S_{10}\left(\gamma_{1}\right) \eta
$$

Indeed, relations (1.17) give

$$
\left[s_{10}(u), s_{10}(v)\right]=-\frac{1}{u+v}\left(s_{1,-1}(u) s_{00}(v)-s_{1,-1}(v) s_{00}(u)\right) \text {. }
$$


Therefore, if $\gamma_{1}+\gamma_{2} \neq 0$ then (6.28) holds because $S_{00}\left(\gamma_{i}\right) \eta=0$. Further, we obtain from (6.29) that

$$
\left[S_{10}(-u), S_{10}(u)\right]=\frac{d S_{1,-1}(u)}{d u} S_{00}(u)-S_{1,-1}(u) \frac{d S_{00}(u)}{d u} .
$$

So, if $\gamma_{1}+\gamma_{2}=0$ then (6.28) holds since in this case $\eta$ is annihilated by both the values of $S_{00}(u)$ and its derivative at $u=\gamma_{2}$.

We suppose, as before, that the $p_{i}$ are nonnegative integers.

Proposition 6.4. The vector $\eta_{p_{1}, \ldots, p_{k}} \in V$ satisfies the relations

$$
\begin{aligned}
S_{-1,1}^{*}(u) \eta_{p_{1}, \ldots, p_{k}} & =0, \\
S_{11}^{*}(u) \eta_{p_{1}, \ldots, p_{k}} & =\left(u-\alpha_{1}^{*}\right) \cdots\left(u-\alpha_{k}^{*}\right)\left(u+\beta_{1}^{*}+p_{1}\right) \cdots\left(u+\beta_{k}^{*}+p_{k}\right) \eta_{p_{1}, \ldots, p_{k}} .
\end{aligned}
$$

Proof. Relation (6.31) follows from (6.17) and Proposition 6.3 by an easy induction; cf. the proof of (6.16). The proof of (6.30) is the same with the use of (6.12) and the expansion for $\left[\widehat{s}_{-1,1}(u), s_{10}(v)\right]$ given by $(1.28)$.

Recall that the representation $L(\gamma, \gamma, \delta)$ of $\mathrm{Y}(3)$ if finite-dimensional if and only if $\gamma-\delta \in \mathbb{Z}_{+}$; see Section 2 .

Proposition 6.5. Let the irreducible representation $V(\mu(u))$ of $\mathrm{Y}^{+}(3)$ with the components of $\mu(u)$ given by (6.4) be finite-dimensional and let $\widetilde{\mu}(u)$ be defined by (6.4) with the corresponding parameters $\widetilde{\alpha}_{i}$ and $\widetilde{\beta}_{i}$, where

$$
\widetilde{\alpha}_{i}=\alpha_{i}+l_{i}, \quad \widetilde{\beta}_{i}=\beta_{i}-m_{i}, \quad i=1, \ldots, k
$$

and $l_{i}, m_{i} \in \mathbb{Z}_{+}$. Then the representation $V(\widetilde{\mu}(u))$ is also finite-dimensional.

Proof. For each $i$ the representation $L\left(\alpha_{i}+l_{i}, \alpha_{i}+l_{i}, \alpha_{i}\right) \otimes V(\mu(u))$ of $\mathrm{Y}^{+}(3)$ is finitedimensional. The tensor product of the highest weight vectors of $L\left(\alpha_{i}+l_{i}, \alpha_{i}+l_{i}, \alpha_{i}\right)$ and $V(\mu(u))$ generates a $\mathrm{Y}^{+}(3)$-module with the highest weight $\mu^{\prime}(u)$, where

$$
\begin{aligned}
& \mu_{0}^{\prime}(u)=\mu_{0}(u)\left(1-\left(\alpha_{i}+l_{i}\right)^{2} u^{-2}\right), \\
& \mu_{1}^{\prime}(u)=\mu_{1}(u)\left(1-\left(\alpha_{i}+l_{i}\right) u^{-1}\right)\left(1+\alpha_{i} u^{-1}\right)
\end{aligned}
$$

see (1.31) and (3.11). The composition of this module and the automorphism (1.29) with the series $\psi(u)=\left(1-\alpha_{i}^{2} u^{-2}\right)^{-1}$ yields a finite-dimensional representation with the highest weight obtained from $\mu(u)$ by replacing $\alpha_{i}$ with $\alpha_{i}+l_{i}$.

Similarly, considering tensor products of the form $L\left(\beta_{i}, \beta_{i}, \beta_{i}-m_{i}\right) \otimes V(\mu(u))$ we show that the representation with the highest weight obtained from $\mu(u)$ by replacing $\beta_{i}$ with $\beta_{i}-m_{i}$ if also finite-dimensional.

We are now in a position to establish necessary and sufficient conditions for $V(\mu(u))$ to be finite-dimensional. 
Proposition 6.6. The irreducible highest weight representation $V(\mu(u))$ of $\mathrm{Y}^{+}(3)$ with the components of $\mu(u)$ given by (6.4) is finite-dimensional if and only if there exist re-enumerations of the $\alpha_{i}$ and $\beta_{i}$ such that either one of the following two conditions is satisfied

$$
\begin{aligned}
& \alpha_{i}-\beta_{i} \in \mathbb{Z}_{+} \quad \text { for } \quad i=1, \ldots, k \\
& \alpha_{i}-\beta_{i} \in \mathbb{Z}_{+} \quad \text { for } \quad i=1, \ldots, k-1 \quad \text { and } \quad \alpha_{k} \in \frac{1}{2}+\mathbb{Z}_{+}, \quad \beta_{k} \in-\mathbb{Z}_{+} .
\end{aligned}
$$

Proof. Suppose that $\operatorname{dim} V(\mu(u))<\infty$. As before, we equip the vector space $V=V(\mu(u))$ with one more structure of $\mathrm{Y}^{+}(3)$-module. This module is isomorphic to $V\left(\mu^{*}(u)\right)$ with $\mu^{*}(u)$ given by $(6.11)$. Our basic idea is to construct nonzero vectors which generate highest weight $\mathrm{Y}^{+}(2)$-submodules in $V\left(\mu^{*}(u)\right)$ and then use the conditions on the highest weight imposed by Proposition 5.3.

Consider the mutliset $M=\left\{\alpha_{1}^{*}, \ldots, \alpha_{k}^{*}, \beta_{1}^{*}, \ldots, \beta_{k}^{*}\right\}$. Let us represent it as a disjoint union

$$
M=\bigsqcup_{z \in \mathbb{C} / \mathbb{Z}} M_{z}, \quad M_{z}=(z+\mathbb{Z}) \cap M .
$$

Let

$$
M_{z}=\left\{\alpha_{i_{1}}^{*}, \ldots, \alpha_{i_{r}}^{*}, \beta_{j_{1}}^{*}, \ldots, \beta_{j_{s}}^{*}\right\}
$$

with both sequences written in the increasing order of the real parts, where $I_{z}=$ $\left\{i_{1}, \ldots, i_{r}\right\}$ and $J_{z}=\left\{j_{1}, \ldots, j_{s}\right\}$ are subsets of $\{1, \ldots, k\}$ depending on $z$.

Assume first that for each $M_{z}$ the following conditions are satisfied

$$
\begin{aligned}
& \alpha_{i_{1}}^{*}=\cdots=\alpha_{i_{r}}^{*}, \quad \beta_{j_{1}}^{*}=\cdots=\beta_{j_{s}}^{*}, \quad \operatorname{Re} \beta_{j_{1}}^{*}<0, \\
& \text { and if } \quad \beta_{j^{\prime}}^{*} \in M_{-z}
\end{aligned}
$$

Consider the vector $\eta_{p_{1}, \ldots, p_{k}}$ (see $\left.(6.23)\right)$ with the numbers $p_{i}$ defined as follows. For $M_{z}$ given by (6.35) set $p_{j_{b}}=\alpha_{j_{b}}+\alpha_{j^{\prime}}, b=1, \ldots, s$ if there exists $\beta_{j^{\prime}}^{*} \in M_{-z}$; otherwise set $p_{j_{b}}=0$. Starting with $\eta_{p_{1}, \ldots, p_{k}}$ we shall apply repeatedly appropriate operators $S_{-1,0}^{\natural}\left(\alpha_{i}-q_{i}\right)$ to the vectors of the form $\eta_{q_{1}, \ldots, q_{k}}$ to get the highest weight vector $\xi=\eta_{0, \ldots, 0}$ with a certain coefficient. By conditions (6.36) and the choice of the numbers $p_{i}$ this coefficient is nonzero, as follows from (6.24). This proves that $\eta_{p_{1}, \ldots, p_{k}} \neq 0$.

On the other hand, by Proposition 6.4 the vector $\eta_{p_{1}, \ldots, p_{k}}$ generates a $\mathrm{Y}^{+}(2)$ submodule in $V\left(\mu^{*}(u)\right)$ with the highest weight

$$
\left(1-\alpha_{1}^{*} u^{-1}\right) \cdots\left(1-\alpha_{k}^{*} u^{-1}\right)\left(1+\left(\beta_{1}^{*}+p_{1}\right) u^{-1}\right) \cdots\left(1+\left(\beta_{k}^{*}+p_{k}\right) u^{-1}\right) .
$$

This submodule is finite-dimensional. However, for all $i$ we have $-\beta_{i}^{*}-p_{i}-\frac{1}{2} \notin \mathbb{Z}_{+}$ and for all $i<j$ we have $-\beta_{i}^{*}-p_{i}-\beta_{j}^{*}-p_{j} \notin \mathbb{Z}_{+}$. So, Proposition 5.3 implies that there exist re-enumerations of the $\alpha_{i}^{*}$ and $\beta_{i}^{*}$ such that either

$$
\alpha_{i}^{*}-\beta_{i}^{*} \in \mathbb{Z}_{+} \quad \text { for } \quad i=1, \ldots, k ;
$$


or

$$
\alpha_{i}^{*}-\beta_{i}^{*} \in \mathbb{Z}_{+} \quad \text { for } \quad i=1, \ldots, k-1 ; \quad \text { and } \quad \alpha_{k}^{*} \in \frac{1}{2}+\mathbb{Z}_{+} .
$$

Let now the parameters $\alpha_{i}^{*}$ and $\beta_{i}^{*}$ be arbitrary. We can choose nonnegative integers $l_{i}$ and $m_{i}$ in such a way that conditions (6.36) are satisfied for any $z$ by the shifted parameters $\alpha_{i}^{*}+l_{i}$ and $\beta_{i}^{*}-m_{i}$. Therefore, by Proposition 6.5 either (6.38), or (6.39) with $\mathbb{Z}_{+}$replaced by $\mathbb{Z}$ will still hold.

Let us consider the multisets $M_{z_{0}}$ for different $z_{0}$.

Suppose first that $z_{0} \not \equiv 0, \frac{1}{2} \bmod \mathbb{Z}$. Then in (6.35) $s$ may only be equal to $r$ or $r+1$. Let us prove that

$$
\alpha_{i_{t}}^{*}-\beta_{j_{t}}^{*} \in \mathbb{Z}_{+} \quad \text { for } \quad t=1, \ldots, r .
$$

Indeed, if this condition is violated then there exists an index $m \in\{1, \ldots, r\}$ such that the number of the elements $\beta_{j_{t}}^{*}$ satisfying $\alpha_{i_{m}}^{*}-\beta_{j_{t}}^{*} \in \mathbb{Z}_{+}$is less than $m$. Now we use Proposition 6.5 again. Applying shifts of the form (6.32), if necessary, we may assume that conditions (6.36) are satisfied for all $z \not \equiv z_{0} \bmod \mathbb{Z}$ while for the elements of $M_{z_{0}}$ we have:

$$
\begin{array}{cc}
\alpha_{i_{1}}^{*}=\cdots=\alpha_{i_{m}}^{*}, & \alpha_{i_{m+1}}^{*}=\cdots=\alpha_{i_{r}}^{*}, \\
\beta_{j_{1}}^{*}=\cdots=\beta_{j_{l}}^{*}, & \beta_{j_{l+1}}^{*}=\cdots=\beta_{j_{s}}^{*},
\end{array}
$$

with $\operatorname{Re} \beta_{j_{1}}^{*}<0$ and if $\beta_{j^{\prime}}^{*} \in M_{-z_{0}}$ then $\alpha_{i_{r}}^{*}+\beta_{j^{\prime}}^{*}>0$. Here $l<m$ and the differences $\alpha_{i_{1}}^{*}-\beta_{j_{1}}^{*}, \beta_{j_{s}}^{*}-\alpha_{i_{1}}^{*}$ and $\alpha_{i_{r}}^{*}-\beta_{j_{s}}^{*}$ are positive integers.

Consider the vector $\eta_{p_{1}, \ldots, p_{k}}$ with the $p_{i}$ defined as in the previous argument except for $i \in J_{z_{0}} \cup J_{-z_{0}}$. In the latter case set $p_{j_{b}}=\alpha_{j_{b}}+\alpha_{j^{\prime}}$ for $b=l+1, \ldots, s$ if there exists $\beta_{j^{\prime}}^{*} \in M_{-z_{0}}$; otherwise set $p_{j_{b}}=0$ (shifting further $\beta_{j^{\prime}}^{*} \rightarrow \beta_{j^{\prime}}^{*}-m_{j^{\prime}}$ we may suppose that the $p_{j_{b}}$ are nonnegative integers). Let each of the remaining $p_{i}$ with $i \in J_{z_{0}} \cup J_{-z_{0}}$ be equal to zero. Using again (6.24) we find that $\eta_{p_{1}, \ldots, p_{k}} \neq 0$ and so by Proposition 6.4 it generates a finite-dimensional $\mathrm{Y}^{+}(2)$-submodule in $V\left(\mu^{*}(u)\right)$ with the highest weight given by $(6.37)$. However, one easily checks that the condition of Proposition 5.3 is not satisfied. Contradiction.

Further, if $z_{0} \equiv 0 \bmod \mathbb{Z}$ the same argument shows that (6.40) holds. The only difference is that here $M_{z_{0}}=M_{-z_{0}}$ and one should take $j^{\prime}$ to be equal to $j_{1}$ or $j_{s}$ depending on whether $\beta_{j_{s}}^{*} \in 1+\mathbb{Z}_{+}$or $\beta_{j_{s}}^{*} \in-\mathbb{Z}_{+}$.

Finally, let $z_{0} \equiv \frac{1}{2} \bmod \mathbb{Z}$. In (6.35) $s$ must be equal to $r$ or $r-1$. We shall prove that either condition (6.40) holds (then $s=r$ ), or

$$
\alpha_{i_{t}^{\prime}}^{*}-\beta_{j_{t}}^{*} \in \mathbb{Z}_{+} \quad \text { for } \quad t=1, \ldots, r-1 \quad \text { and } \quad \alpha_{i_{r}^{\prime}}^{*} \in \frac{1}{2}+\mathbb{Z}_{+}
$$

for a permutation $\left(i_{1}^{\prime}, \ldots, i_{r}^{\prime}\right)$ of the elements of $I_{z_{0}}$.

Suppose that (6.40) is violated. Then we can again represent $M_{z_{0}}$ in the form (6.41). If either $\beta_{j_{s}}^{*} \in \frac{3}{2}+\mathbb{Z}_{+}$with $\alpha_{i_{1}}^{*} \in-\frac{1}{2}-\mathbb{Z}_{+}$, or $\beta_{j_{s}}^{*} \in \frac{1}{2}-\mathbb{Z}_{+}$then we come to 
a contradiction exactly as above. So, we must have $\beta_{j_{s}}^{*} \in \frac{3}{2}+\mathbb{Z}_{+}, \quad \alpha_{i_{1}}^{*} \in \frac{1}{2}+\mathbb{Z}_{+}$. Taking $j^{\prime}=j_{1}$ and repeating the previous argument we obtain the inequality $s-l \leq$ $r-m$. It can only be satisfied if $s=r-1$ and $l=m-1$. Note that this conclusion is valid for all possible values of the parameter $m$. Let us now assume that $m$ takes the minimum value. Then $(6.42)$ will be satisfied for the indices $i_{1}^{\prime}, \ldots, i_{r}^{\prime}$ defined as follows:

$$
\begin{array}{ll}
i_{t}^{\prime}=i_{t} & \text { for } \quad t=1, \ldots, m-1, \\
i_{t}^{\prime}=i_{t+1} & \text { for } \quad t=m, \ldots, r-1, \\
i_{r}^{\prime}=i_{m} . &
\end{array}
$$

Thus, we have proved that if $V(\mu(u))$ is finite-dimensional then there exist reenumerations of the $\alpha_{i}$ and $\beta_{i}$ such that either (6.38), or (6.39) holds. Conditions (6.38) are equivalent to (6.33), while (6.39) means that

$$
\alpha_{i}-\beta_{i} \in \mathbb{Z}_{+} \quad \text { for } \quad i=1, \ldots, k-1 ; \quad \text { and } \quad \beta_{k} \in-\mathbb{Z}_{+} ;
$$

see (6.10). Applying this result to the representation $V\left(\mu^{*}(u)\right)$ instead of $V(\mu(u))$ we obtain that either relation (6.33) holds, or there exist permutations $\sigma, \tau \in \mathfrak{S}_{k}$ such that

$$
\alpha_{\sigma(i)}-\beta_{\tau(i)} \in \mathbb{Z}_{+} \quad \text { for } \quad i=1, \ldots, k-1 ; \quad \text { and } \quad \alpha_{\sigma(k)} \in \frac{1}{2}+\mathbb{Z}_{+} .
$$

If $\sigma(k)=k$ then (6.34) is satisfied. Otherwise, set $l=\sigma(k)$ and rewrite (6.44) as follows:

$$
\alpha_{i}-\beta_{\rho(i)} \in \mathbb{Z}_{+} \quad \text { for } \quad i \neq l ; \quad \text { and } \quad \alpha_{l} \in \frac{1}{2}+\mathbb{Z}_{+},
$$

where $\rho \in \mathfrak{S}_{k}$. Consider the cycle of $\rho$ of the form $k \rightarrow i_{1} \rightarrow \cdots \rightarrow i_{s} \rightarrow k$. Suppose that it does not contain $l$. Then setting $\beta_{i}^{\prime}=\beta_{\rho(i)}$ if $i$ belongs to the cycle, and $\beta_{i}^{\prime}=\beta_{i}$ otherwise, we obtain by (6.43) that $\alpha_{i}-\beta_{i}^{\prime} \in \mathbb{Z}_{+}$for all $i=1, \ldots, k$, that is, (6.33) holds.

Suppose now that the cycle contains $l: k \rightarrow i_{1} \rightarrow \cdots \rightarrow i_{t} \rightarrow l$. Set $\alpha_{k}^{\prime}=\alpha_{l}$, $\alpha_{\rho(i)}^{\prime}=\alpha_{i}$ for $i=k, i_{1}, \ldots, i_{t}$ and $\alpha_{i}^{\prime}=\alpha_{i}$ for the remaining indices $i$. Then by (6.43) relation (6.34) is satisfied for the $\alpha_{i}^{\prime}$ and $\beta_{i}$ which completes the proof of the 'only if' part of the proposition.

Now let (6.33) hold. Then representation $(6.5)$ of $\mathrm{Y}^{+}(3)$ is finite-dimensional. However, $V(\mu(u))$ is isomorphic to its subquotient and therefore $\operatorname{dim} V(\mu(u))<\infty$.

Finally, let (6.34) hold. Consider the highest weight representation $V(\mu)$ of the Lie algebra $\mathfrak{o}(3)$ and extend it to $\mathrm{Y}^{+}(3)$ by using the homomorphism (1.23). We obtain a representation with the highest weight given by (3.10). By (3.9) the representation $V(\mu)$ is finite-dimensional for $\mu=\beta_{k}-\frac{1}{2}$. Therefore the representation of $\mathrm{Y}^{+}(3)$ of the form

$$
L\left(\alpha_{1}, \alpha_{1}, \beta_{1}\right) \otimes \cdots \otimes L\left(\alpha_{k-1}, \alpha_{k-1}, \beta_{k-1}\right) \otimes L\left(\alpha_{k}, \alpha_{k}, \frac{1}{2}\right) \otimes V\left(\beta_{k}-\frac{1}{2}\right)
$$


is also finite-dimensional. However, we find from (1.31) and $(3.11)$ that $V(\mu(u))$ is isomorphic to a subquotient of $(6.45)$ and so, $\operatorname{dim} V(\mu(u))<\infty$.

The following theorem together with Theorem 3.3 gives a description of finitedimensional irreducible representations of $\mathrm{Y}^{+}(2 n+1)$ (cf. Theorems 4.8 and 5.9). We use notation (2.35).

Theorem 6.7. The irreducible highest weight representation $V(\mu(u)), \mu(u)=$ $\left(\mu_{0}(u), \ldots, \mu_{n}(u)\right)$ of $\mathrm{Y}^{+}(2 n+1)$ is finite-dimensional if and only if either one of the following two relations holds:

$$
\begin{aligned}
\mu_{0}(u) & \rightarrow \mu_{1}(u) \rightarrow \cdots \rightarrow \mu_{n}(u), \\
\frac{2 u}{2 u+1} \mu_{0}(u) & \rightarrow \mu_{1}(u) \rightarrow \cdots \rightarrow \mu_{n}(u) .
\end{aligned}
$$

Proof. Suppose that $\operatorname{dim} V(\mu(u))<\infty$. Then by Proposition 3.5 we have

$$
\mu_{1}(u) \rightarrow \mu_{2}(u) \rightarrow \cdots \rightarrow \mu_{n}(u)
$$

The subalgebra in $\mathrm{Y}^{+}(2 n+1)$ generated by the coefficients of the series $s_{i j}(u)$ with $i, j=-1,0,1$ is isomorphic to $\mathrm{Y}^{+}(3)$. The cyclic span of the highest weight vector of $V(\mu(u))$ with respect to this subalgebra is a representation with the highest weight $\left(\mu_{0}(u), \mu_{1}(u)\right)$. Its irreducible quotient is finite-dimensional and so, by Proposition 6.6 we have $\mu_{0}(u) \rightarrow \mu_{1}(u)$ or $\frac{2 u}{2 u+1} \mu_{0}(u) \rightarrow \mu_{1}(u)$ depending on whether condition (6.33) or (6.34) is satisfied; see the proof of Theorem 2.8.

Conversely, let relation (6.46) hold. Then for $i=1, \ldots, n$ we have

$$
\frac{\mu_{i-1}(u)}{\mu_{i}(u)}=\frac{P_{i}(u+1)}{P_{i}(u)}
$$

for monic polynomials $P_{i}(u)$. Let

$$
P_{i}(u)=\left(u+\delta_{1}^{(i)}\right) \cdots\left(u+\delta_{s_{i}}^{(i)}\right), \quad i=1, \ldots, n
$$

Consider the irreducible highest weight representation $L(\lambda(u))$ with $\lambda(u)=$ $\left(\lambda_{-n}(u), \ldots, \lambda_{n}(u)\right)$ of the Yangian $\mathrm{Y}(2 n+1)$ where the $\lambda_{i}(u)$ are defined by $(4.42)$ for $i=1, \ldots, n$ and by $(4.43)$ for $i=0, \ldots, n$. Then $L(\lambda(u))$ is finite-dimensional by Theorem 2.12 and we conclude by repeating the corresponding argument of the proof of Theorem 4.8.

Finally, let (6.47) hold. Then there exist monic polynomials (6.49) such that (6.48) holds for $i=2, \ldots, n$ and

$$
\frac{\mu_{0}(u)}{\mu_{1}(u)}=\frac{P_{1}(u+1)}{P_{1}(u)} \frac{2 u+1}{2 u} .
$$


Let $V\left(\mu_{0}\right)$ denote the irreducible representation of the Lie algebra $\mathfrak{o}(2 n+1)$ with the highest weight $\mu_{0}=(-1 / 2, \ldots,-1 / 2)$. It is finite-dimensional; see (3.6) and (3.9). Extend $V\left(\mu_{0}\right)$ to a representation of $\mathrm{Y}^{+}(2 n+1)$ by using $(1.23)$ and consider the tensor product $L(\lambda(u)) \otimes V\left(\mu_{0}\right)$, where $L(\lambda(u))$ is the defined above representation of $\mathrm{Y}(2 n+1)$. The tensor product of the highest weight vectors of $L(\lambda(u))$ and $V\left(\mu_{0}\right)$ generates a $\mathrm{Y}^{+}(2 n+1)$-submodule with the highest weight $\mu^{\prime}(u)=\left(\mu_{0}^{\prime}(u), \ldots, \mu_{n}^{\prime}(u)\right)$ where $\mu_{i}^{\prime}(u)=\lambda_{i}(u) \lambda_{-i}(-u)\left(1+1 / 2 u^{-1}\right)^{-1}$ for $i=$ $1, \ldots, n$ and $\mu_{0}^{\prime}(u)=\lambda_{0}(u) \lambda_{0}(-u)$; see (1.31) and (3.11). So the representation $V\left(\mu^{\prime}(u)\right)$ of $\mathrm{Y}^{+}(2 n+1)$ is finite-dimensional and the $\mu_{i}^{\prime}(u)$ satisfy $(6.48)$ for $i=$ $2, \ldots, n$ and $(6.50)$. It remains to repeat the corresponding argument of the proof of Theorem 5.9 .

Theorem 6.7 implies the following parameterization of representations of the special twisted Yangian $\mathrm{SY}^{+}(2 n+1)$ (cf. Corollaries 4.9 and 5.10).

Corollary 6.8. There is a one-to-one correspondence between finite-dimensional irreducible representations of the special twisted Yangian $\mathrm{SY}^{+}(2 n+1)$ and the families $\left\{P_{1}(u), \ldots, P_{n}(u), \varepsilon\right\}$, where the $P_{i}(u)$ are monic polynomials in $u$ and the parameter $\varepsilon$ takes values in $\{1,2\}$. Every such representation is isomorphic to a subquotient of a representation of the form $L(\lambda(u))$ or $L(\lambda(u)) \otimes V\left(\mu_{0}\right)$.

Remark 6.9. One can use Propositions 6.3 and 6.4 to get a 'branching rule' for the restriction of a 'generic' finite-dimensional irreducible representation $V$ of $\mathrm{Y}^{+}(3)$ to the subalgebra $\mathrm{Y}^{+}(2)$ and thus to construct an analogue of Gelfand-Tsetlin basis in $V$; cf. [M2], [NT2].

\section{References}

[BL] D. Bernard and A. LeClair, Quantum group symmetries and non-local currents in 2D QFT. Commun. Math. Phys. 142 (1991), 99-138.

[CP1] V. Chari and A. Pressley, Yangians and $R$-matrices, L'Enseign. Math. 36 (1990), 267-302.

[CP2] V. Chari and A. Pressley, Fundamental representations of Yangians and singularities of $R$-matrices, J. Reine Angew. Math. 417 (1991), 87-128.

[CP3] V. Chari and A. Pressley, Yangians: their representations and characters, Acta Appl. Math. 44 (1996), 39-58.

[C1] I. V. Cherednik, A new interpretation of Gelfand-Tzetlin bases, Duke Math. J. 54 (1987), 563-577.

[C2] I. V. Cherednik, Quantum groups as hidden symmetries of classic representation theory, in 'Differential Geometric Methods in Physics (A. I. Solomon, Ed.)', World Scientific, Singapore, 1989, pp. 47-54.

[Di] J. Dixmier, Algèbres Enveloppantes, Gauthier-Villars, Paris, 1974. 
[D1] V. G. Drinfeld, Hopf algebras and the quantum Yang-Baxter equation, Soviet Math. Dokl. 32 (1985), 254-258.

[D2] V. G. Drinfeld, A new realization of Yangians and quantized affine algebras, Soviet Math. Dokl. 36 (1988), 212-216.

[KR] A. N. Kirillov and N. Yu. Reshetikhin, Yangians, Bethe ansatz and combinatorics, Lett. Math. Phys. 12 (1986), 199-208.

[KK] T. H. Koornwinder and V. B. Kuznetsov, Gauss hypergeometric function and quadratic R-matrix algebras, St. Petersburg Math. J. 6 (1994), 161-184.

[K] V. E. Korepin, The analysis of the bilinear relation of the six-vertex model, Soviet Phys. Dokl. 27 (1982), 612-613.

[KS1] P. P. Kulish and E. K. Sklyanin, Quantum spectral transform method: recent developments, in 'Integrable Quantum Field Theories', Lecture Notes in Phys. 151 Springer, Berlin-Heidelberg, 1982, pp. 61-119.

[KS2] P. P. Kulish and E. K. Sklyanin, Algebraic structures related to reflection equations, J. Phys. A25 (1992), 5963-5975.

[KJC] V. B. Kuznetsov, M. F. Jørgensen, P. L. Christiansen, New boundary conditions for integrable lattices, J. Phys. A 28 (1995), 4639.

[M1] A. Molev, Representations of twisted Yangians, Lett. Math. Phys. 26 (1992), 211-218.

[M2] A. Molev, Gelfand-Tsetlin basis for representations of Yangians, Lett. Math. Phys. 30 (1994), 53-60.

[M3] A. Molev, Sklyanin determinant, Laplace operators, and characteristic identities for classical Lie algebras, J. Math. Phys. 36 (1995), 923-943.

[M4] A. Molev, Noncommutative symmetric functions and Laplace operators for classical Lie algebras, Lett. Math. Phys. 35 (1995), 135-143.

[MN] A. Molev and M. Nazarov, Capelli identities for classical Lie algebras, Preprint CMA 003-97, Australian National University, Canberra.

[MNO] A. Molev, M. Nazarov and G. Olshanski, Yangians and classical Lie algebras, Russian Math. Surveys 51:2 (1996), 205-282.

[N1] M. Nazarov, Quantum Berezinian and the classical Capelli identity, Lett. Math. Phys. 21 (1991), 123-131.

[N2] M. Nazarov, Yangians and Capelli identities, in "A. A. Kirillov Seminar on Representation Theory", AMS, Providence, 1997, pp 139-164; q-alg/9601027.

[NO] M. Nazarov and G. Olshanski, Bethe subalgebras in twisted Yangians, Commun. Math. Phys. 178 (1996), 483-506.

[NT1] M. Nazarov and V. Tarasov, Yangians and Gelfand-Zetlin bases, Proc. RIMS, Kyoto Univ., 30 (1994), 459-478.

[NT2] M. Nazarov and V. Tarasov, Representations of Yangians with Gelfand-Zetlin bases, to appear in J. Reine Angew. Math.; q-alg/9502008.

[Ok] A. Okounkov, Quantum immanants and higher Capelli identities, Transformation Groups 1 (1996), 99-126.

[O1] G. Olshanski, Representations of infinite-dimensional classical groups, limits of enveloping algebras, and Yangians, in 'Topics in Representation Theory (A. 
A. Kirillov, Ed.), Advances in Soviet Math. 2, AMS, Providence RI, 1991, pp. $1-66$.

[O2] G. Olshanski, Twisted Yangians and infinite-dimensional classical Lie algebras, in 'Quantum Groups (P. P. Kulish, Ed.)', Lecture Notes in Math. 1510, Springer, Berlin-Heidelberg, 1992, pp. 103-120.

[S] E. K. Sklyanin, Boundary conditions for integrable quantum systems, J. Phys. A21 (1988), 2375-2389.

[TU] K. Takemura and D. Uglov, The orthogonal eigenbasis and norms of eigenvectors in the Spin Calogero-Sutherland Model, Preprint RIMS 1114, Kyoto, 1996; solv-int/9611006.

[TF] L. A. Takhtajan and L. D. Faddeev, Quantum inverse scattering method and the Heisenberg XYZ-model, Russian Math. Surv. 34 (1979), no. 5, 11-68.

[T1] V. O. Tarasov, Structure of quantum L-operators for the R-matrix of of the XXZ-model, Theor. Math. Phys. 61 (1984), 1065-1071.

[T2] V. O. Tarasov, Irreducible monodromy matrices for the $R$-matrix of the $X X Z$ model and lattice local quantum Hamiltonians, Theor. Math. Phys. 63 (1985), 440-454.

[UK] D. B. Uglov and V. E. Korepin, The Yangian symmetry of the Hubbard model, Phys. Lett. A 190 (1994), 238-242.

[Z1] R. B. Zhang, Representations of super Yangian, J. Math. Phys. 36 (1995), no. $7,3854-3865$.

[Z2] R. B. Zhang, The $\mathfrak{g l}(M \mid N)$ super Yangian and its finite-dimensional representations, Lett. Math. Phys. 37 (1996), 419-434. 